TRANSACTIONS OF THE

AMERICAN MATHEMATICAL SOCIETY

Volume 350, Number 12, December 1998, Pages 4709-4756

S $0002-9947(98) 02334-4$

\title{
ON THE EXISTENCE OF MULTIPLE STEADY-STATE SOLUTIONS IN THE THEORY OF ELECTRODIFFUSION. PART I: THE NONELECTRONEUTRAL CASE. PART II: A CONSTRUCTIVE METHOD FOR THE ELECTRONEUTRAL CASE
}

\author{
FATIHA ALABAU
}

\begin{abstract}
We give a constructive method for giving examples of doping functions and geometry of the device for which the nonelectroneutral voltage driven equations have multiple solutions. We show in particular, by performing a singular perturbation analysis of the current driven equations that if the electroneutral voltage driven equations have multiple solutions then the nonelectroneutral voltage driven equations have multiple solutions for sufficiently small normed Debye length. We then give a constructive method for giving examples of data for which the electroneutral voltage driven equations have multiple solutions.
\end{abstract}

\section{INTRODUCTION}

The theory of electrodiffusion arises in the study of transport of charges in devices which are generally involved in electrical engineering (semiconductors) or electrochemistry (ion exchangers, ... . ). An essential feature of this theory is that the transport of charges is assumed to result from the combined effect of diffusion (due to the gradient of concentration of particles) and drift (due to the electric field) processes. This type of modelization is valid as long as the size of the device is not below the micron; otherwise more sophisticated models such as hydrodynamic ones or even Boltzmann's equations have to be used.

The electrodiffusion models consist of parameter dependent nonlinear systems of coupled differential equations of Poisson and convection-diffusion type. The parameter (which is in general a vector in the multi-dimensional case) represents the external forces acting on the device. The components of this vector are the differences of potential (or biases) applied at the contacts of the device in the case of the voltage driven models, whereas they are the currents flowing through the contacts in the case of the current driven models. The device is essentially characterized by its geometry, by the doping function which appears as an inhomogeneity in the Poisson's equation and by other physical scalar parameters (such as Debye length and intrinsic concentration) which appear in the equations or in the boundary conditions.

Received by the editors November 30, 1995.

1991 Mathematics Subject Classification. Primary 35G30, 35J25, 35B50.

Key words and phrases. Semiconductor, electrochemistry, nonlinear system, elliptic, existence, multiplicity. 
Both parts of this paper are concerned with an analysis of the respective roles of the doping inhomogeneity and of certain physical parameters on the existence of multiple steady-state solutions of the voltage driven drift-diffusion model (we will specify later in part II which physical parameters are important for our purposes). This fundamental model has been derived in 1950 by Van Roosbroeck [14]. It has been intensively studied from a mathematical point of view (see e.g. [11, 16, 13, 10, $15]$ and the references therein) for many years, and various results on the existence and qualitative properties of solutions for the steady-state and non-steady-state problem have been obtained. The situation is very different as far as uniqueness or multiplicity of the steady-state solutions is concerned. It is indeed striking (and many authors, see e.g. $[8,13,15]$ have emphasized this lack) that for many years no multiplicity results have been proved, even in the one-dimensional case.

Indeed, it is well known that, from physical grounds (see e.g. [18]), uniqueness does not hold in general. In particular it is conjectured that if the doping inhomogeneity has at least three alterations of sign in the device then multiplicity can occur (see e.g. $[12,15]$ ). Also, several numerical examples of multiplicity in the one-dimensional case and for doping inhomogeneities having three alterations of signs (case of thyristors) can be found in the literature (see e.g. $[12,15,17]$ ). On the other side, positive uniqueness results have been obtained mainly in the following situations: either the applied voltages are assumed to be sufficiently small while no restrictions are made on the doping inhomogeneity (see e.g. $[13,10]$ ), or the applied voltage is assumed to be arbitrarily large but specific assumptions (in particular on the number of sign alterations of the doping inhomogeneity) are made $[2,3,5]$.

Hence from a rigorous mathematical point of view the question of multiplicity is still open, and, even more precisely, the mathematical mechanisms which could explain (even without proofs) the occurrence of multiple steady-state solutions are far from being understood. The purpose of the present paper is to answer this question and to explain which mechanisms are responsible for multiplicity in the one dimensional case. As will be seen in the course of this paper, the answer is complex, mainly because several parameters as well as the doping inhomogeneity are involved in different ways.

Before we state more precisely the main results of this paper, we give below the (scaled) voltage driven drift-diffusion equations.

We assume that the mobilities are constant and that the semiconductor is not degenerate, so that the Einstein's relations are valid. Moreover, we assume that there is no generation recombination. Under these assumptions and for a prescribed voltage $V$ in $\mathbb{R}$, the fundamental voltage driven drift-diffusion equations are, in dimensionless form (see [14]),

$$
\begin{gathered}
\varepsilon \psi_{\varepsilon}^{\prime \prime}=n_{\varepsilon}-p_{\varepsilon}-N \text { in } \Omega=(-1,1), \\
n_{\varepsilon}^{\prime}-n_{\varepsilon} \psi_{\varepsilon}^{\prime}=J_{n, \varepsilon} \text { in } \Omega, \\
p_{\varepsilon}^{\prime}+p_{\varepsilon} \psi_{\varepsilon}^{\prime}=J_{n, \varepsilon}-I_{\varepsilon} \text { in } \Omega, \\
J_{n, \varepsilon}^{\prime}=0 \text { in } \Omega, \\
I_{\varepsilon}^{\prime}=0 \text { in } \Omega,
\end{gathered}
$$

subject to the boundary conditions

$$
\psi_{\varepsilon}(-1)=\psi_{-1},
$$




$$
\begin{gathered}
\psi_{\varepsilon}(1)=\log \left(\frac{n_{1}}{n_{-1}}\right)+\psi_{-1}-V . \\
n_{\varepsilon}( \pm 1)=n_{ \pm 1}>0, \quad p_{\varepsilon}( \pm 1)=p_{ \pm 1}>0
\end{gathered}
$$

where

$$
n_{-1} p_{-1}=n_{1} p_{1}=\delta^{4} .
$$

System (1.1)-(1.9) models the transport of electrons and holes in a semiconductor device. The unknowns are the functions $\psi_{\varepsilon}, n_{\varepsilon}, p_{\varepsilon}$ and the numbers $J_{n, \varepsilon}$ and $I_{\varepsilon}$, which stand respectively for the electrostatic potential, the electron and hole densities and for the electron current and total current density. The number $I_{\varepsilon}-J_{n, \varepsilon}$ and the function $-\psi_{\varepsilon}^{\prime}$ represent respectively the hole current density and the electric field. The doping profile $N$, which is a given function defined on $\bar{\Omega}$, as well as the positive numbers $\varepsilon$ and $\delta$, depend only on the semiconductor device and not on the parameter $V$.

Since the nonlinear system (1.1)-(1.9) depends on the parameters $V$ and $\varepsilon$, it is denoted from now on by $(\mathrm{VD})_{V, \varepsilon}$.

The current driven model is formed by (1.1)-(1.4), (1.6) and (1.8)-(1.9), where $I_{\varepsilon}$ has to be replaced by $I$. Since this system depends on $I$ and $\varepsilon$, it is denoted from now on by $(\mathrm{CD})_{I, \varepsilon}$. The unknowns of this system are $\psi_{\varepsilon}, n_{\varepsilon}, p_{\varepsilon}$ and $J_{n, \varepsilon}$.

We recall that the existence of weak solutions $\left(\psi_{\varepsilon}, n_{\varepsilon}, p_{\varepsilon}, J_{n, \varepsilon}, I_{\varepsilon}\right)$ of $(\mathrm{VD})_{V, \varepsilon}$ has been proved for arbitrary $V$ in $\mathbb{R}$ by many authors (see e.g [10] and the references therein) and that any solution $\left(\psi_{\varepsilon}, n_{\varepsilon}, p_{\varepsilon}, J_{n, \varepsilon}, I_{\varepsilon}\right)$ of $(\mathrm{VD})_{V, \varepsilon}$ satisfies

$$
0<n_{\varepsilon}, \quad 0<p_{\varepsilon} .
$$

We also recall that existence and uniqueness of solutions of $(\mathrm{CD})_{I, \varepsilon}$, as well as analyticity of the solution with respect to $I$, is proved in [4]. Of course in the case of the current driven model the voltage $V_{\varepsilon}$ is unknown and is given by

$$
V_{\varepsilon}=\log \left(\frac{n_{1}}{n_{-1}}\right)+\psi_{-1}-\psi_{\varepsilon}(1) .
$$

where $\psi_{\varepsilon}$ is the electrostatic potential corresponding to the (unique) solution of $(\mathrm{CD})_{I, \varepsilon}$. We also proved in [4] that the voltage function $\mathcal{V}_{\varepsilon}$ defined by

$$
I \mapsto V_{\varepsilon}
$$

is well defined, onto and satisfies

$$
\mathcal{V}_{\varepsilon}(I) \rightarrow \pm \infty \quad \text { if and only if } \quad I \rightarrow \pm \infty .
$$

The current and voltage driven systems are related formally in the following way:

Assume that $I$ is given and let $\left(\psi_{\varepsilon}, n_{\varepsilon}, p_{\varepsilon}, J_{n, \varepsilon}\right)$ be a solution of $(\mathrm{CD})_{I, \varepsilon}$. Then $\left(\psi_{\varepsilon}, n_{\varepsilon}, p_{\varepsilon}, J_{n, \varepsilon}, I_{\varepsilon}\right)$ is a solution of $(\mathrm{VD})_{V_{\varepsilon}, \varepsilon}$, where $V_{\varepsilon}$ is given by (1.10). Conversely, assume that $V$ is given and let $\left(\psi_{\varepsilon}, n_{\varepsilon}, p_{\varepsilon}, J_{n, \varepsilon}, I_{\varepsilon}\right)$ be a solution of $(\mathrm{VD})_{V, \varepsilon}$. The equations (1.2) and (1.3) can be written under the form

$$
\left(n_{\varepsilon} \exp \left(-\psi_{\varepsilon}\right)\right)^{\prime}=J_{n, \varepsilon} \exp \left(-\psi_{\varepsilon}\right),
$$

and

$$
\left(p_{\varepsilon} \exp \left(\psi_{\varepsilon}\right)\right)^{\prime}=\left(J_{n, \varepsilon}-I_{\varepsilon}\right) \exp \left(\psi_{\varepsilon}\right)
$$


Integration from $x=-1$ to $x=1$ of these two last equations together with (1.4)(1.10), gives

$$
\begin{aligned}
I_{\varepsilon}= & n_{-1} \exp \left(-\psi_{-1}\right)(\exp (V)-1)\left(\int_{\Omega} \exp \left(-\psi_{\varepsilon}\right) d x\right)^{-1}+p_{-1} \exp \left(\psi_{-1}\right) \\
& \times(1-\exp (-V))\left(\int_{\Omega} \exp \left(\psi_{\varepsilon}\right) d x\right)^{-1} .
\end{aligned}
$$

Hence, $\left(\psi_{\varepsilon}, n_{\varepsilon}, p_{\varepsilon}, J_{n, \varepsilon}\right)$ is a solution of $(\mathrm{CD})_{I_{\varepsilon}, \varepsilon}$, where $I_{\varepsilon}$ is given by (1.12).

We also recall that the voltage current characteristic is associated to the voltage driven model. It is the (possibly multi-valued) map

$$
V \mapsto I_{\varepsilon}
$$

where $I_{\varepsilon}$ is the total current associated to a solution of $(\mathrm{VD})_{V, \varepsilon}$.

As we already said, the purpose of this paper is to give conditions on the data associated to $(\mathrm{VD})_{V, \varepsilon}$ which are sufficient to guarantee the existence of multiple solutions of $(\mathrm{VD})_{V, \varepsilon}, V$ being the bifurcation parameter. This goal is achieved by analyzing the respective roles of the doping inhomogeneity $N$ and of the physical parameters involved in $(\mathrm{VD})_{V, \varepsilon}$.

Part I is devoted to the analysis of the role of the normed Debye length $\varepsilon$, whereas the other parameters (except $V$ ) as well as the doping inhomogeneity $N$ are kept fixed and arbitrary.

Indeed, since $\varepsilon$ is a small parameter which multiplies the highest derivative in equation (1.1), both systems $(\mathrm{CD})_{I, \varepsilon}$ and $(\mathrm{VD})_{V, \varepsilon}$ can be analyzed in the framework of singular perturbation theory (see e.g. $[9,10,1,6]$ ). Hence we can associate to $(\mathrm{CD})_{I, \varepsilon}\left(\right.$ resp. $\left.(\mathrm{VD})_{V, \varepsilon}\right)$ a reduced current (resp. voltage) driven system $(\mathrm{RCD})_{I}$ $\left(\right.$ resp. $\left.(\mathrm{RVD})_{V}\right)$. These reduced systems are in a formal way the limit problems, as $\varepsilon$ goes to 0 , of the associated singularly perturbed systems. They are introduced in subsection 2.1.

The main result of Part I concerning the role of $\varepsilon$ on the existence of multiple solutions shows that in order to prove the existence of multiple solutions of $(\mathrm{VD})_{V, \varepsilon}$ for certain values of $V$, it is sufficient to prove that the limit problem (RVD) $V$ has multiple (isolated) solutions for certain values of $V$ (provided that $\varepsilon$ is sufficiently small). More precisely, we prove that if the data $N, \delta$ and $V_{0}$ are such that the limit problem (RVD) $V_{0}$ has $k$ isolated solutions, then for $\varepsilon$ sufficiently small there exist $V_{-, \varepsilon}<V_{0}<V_{+, \varepsilon}$ such that for all $\bar{V}$ in $\left(V_{-, \varepsilon}, V_{+, \varepsilon}\right)$, (VD $)_{V, \varepsilon}$ has at least $k$ solutions (see theorem 3.4). We also establish a corollary showing that if the reduced current driven system has a left positive and a right positive (resp. left negative and right negative) saturation current (see the definition in subsection 2.2) then for $\varepsilon$ sufficiently small there exist $V_{-, \varepsilon}<V_{+, \varepsilon}$ such that for all $\bar{V}$ in $\left(V_{-, \varepsilon}, V_{+, \varepsilon}\right),(\mathrm{VD})_{V, \varepsilon}$ has at least three solutions for $\varepsilon$ sufficiently small (see corollary 3.5). These results are valid under very general assumptions on $N$ and $\delta$. Of course such results are of practical interest only if we can prove that there exist data $N, \delta$ and $V_{0}$ such that $(\mathrm{RVD})_{V_{0}}$ has multiple (isolated) solutions. This question is the subject of Part II. The proof of the above results is based on a singular perturbation analysis of the current driven system $(\mathrm{CD})_{I, \varepsilon}$ (see section 2), and on a fundamental uniform estimate (with respect to $\varepsilon$ ) of the inverse of the linearized current driven equations in 
appropriate spaces (see corollary 3.2 and also theorem 3.1). The proof of this fundamental estimate is based on a structural property of the current driven equations which lies in the specific form of the coupling between the two convection-diffusion equations (1.2)-(1.3) and the Poisson equation (1.1). Thanks to this fundamental estimate we prove the main result of this paper concerning the singular perturbation analysis of the current driven equations. This result states that the asymptotic error, which is the difference between the solution of the current driven equations and the first term of an associated modified asymptotic expansion (in the sense of singular perturbation theory), is of order $\sqrt{\varepsilon}$. A similar result holds for the derivatives with respect to $I$ of this asymptotic error. This modified asymptotic expansion is defined in subsection 2.4 (for the values of $I$ for which the reduced current driven equations have a solution). It involves the solution of the reduced current driven equations, together with classical boundary layer and non-classical interface layer terms.

It should be noted at this stage that as far as the multiplicity question for the reduced voltage driven equations is concerned, it is not possible to use the results of [15] and [17] in order to answer this question positively. Effectively, both authors have analyzed the problem of the existence of at least two positive saturation currents for a scalar equation satisfied by the unknown $n+p$, where $n$ and $p$ denote respectively the reduced electron and hole concentrations. This scalar equation is associated to the reduced current driven system in the following way. If $\left(\psi, n, p, J_{n}\right)$ is a solution of $(\mathrm{RCD})_{I}$, then $n+p$ is a solution of this scalar equation. Conversely if $s$ is a (strictly positive) solution of this scalar equation, then one can easily associate to $s$ functions $\psi, n$ and $p$ and a number $J_{n}$, but unfortunately $\left(\psi, n, p, J_{n}\right)$ is not necessarily an admissible solution of $(\mathrm{RCD})_{I}$. Indeed, it can easily be shown that $\left(\psi, n, p, J_{n}\right)$ is a solution of $(\mathrm{RCD})_{I}$ if and only if $s$ satisfies the constraint $s(x)>|N(x)|$ for all $x$ in $\bar{\Omega}$. Hence the existence of at least two positive saturation currents for this scalar equation is not sufficient in order to derive multiplicity results for the reduced voltage driven equations. Moreover it should also be noted that the boundary data under consideration in [15] do not satisfy the electroneutrality condition; hence the system considered in [15] is not the reduced voltage driven system in the classical sense of singular perturbation theory.

We use in all of this paper the following notation

For $m \in \mathbb{N}$ and $q \in N^{\star} \cup\{+\infty\}$ we denote by $W^{m, q}(\Omega)$ the usual Sobolev space equipped with its standard norm \|\|$_{m, q, \Omega}$. For $m=0$ we have $W^{0, q}(\Omega)=$ $L^{q}(\Omega)$. For a given partition $\mathcal{P}_{M}=\left(a_{i}\right)_{0 \leq i \leq M+1}$ (independent on $\varepsilon$ ) in subintervals $\left[a_{i}, a_{i+1}\right], i \in\{0, \ldots, M\}$, and for $(m, q) \in \mathbb{N}^{\star} \times\left(\mathbb{N}^{\star} \cup\{+\infty\}\right)$, we set

$$
W_{d}^{m, q}\left(\Omega, \mathcal{P}_{M}\right)=\left\{u, u_{/\left[a_{i}, a_{i+1}\right]} \in W^{m, q}\left(a_{i}, a_{i+1}\right), i \in\{0, \ldots, M\}\right\},
$$

$$
\begin{aligned}
& W^{m, q}\left(\Omega, \mathcal{P}_{M}\right) \\
& \quad=\left\{u \in W^{m-1, q}(\Omega),\left(u_{/\left[a_{i}, a_{i+1}\right]}\right)^{(m-1)} \in W^{1, q}\left(a_{i}, a_{i+1}\right), i \in\{0, \ldots, M\}\right\} .
\end{aligned}
$$

This last space is a Banach space for the norm defined by

$$
\|u\|_{m, q, \Omega, \mathcal{P}_{M}}=\|u\|_{m-1, q, \Omega}+|u|_{m, q, \Omega, \mathcal{P}_{M}}
$$

where

$$
|u|_{m, q, \Omega, \mathcal{P}_{M}}=\sum_{i=0}^{i=M}\left\|\left(u_{/\left[a_{i}, a_{i+1}\right]}\right)^{(m)}\right\|_{0, q,\left[a_{i}, a_{i+1}\right]}
$$


Moreover we denote by $\mathcal{A}([a, b])$ the set of analytic functions on a given bounded interval $[a, b]$ of $\mathbb{R}$, and we set $\mathcal{A}\left(\bar{\Omega}, \mathcal{P}_{M}\right)=\left\{u,\left(u_{/\left[a_{i}, a_{i+1}\right]}\right) \in \mathcal{A}\left(\left[a_{i}, a_{i+1}\right]\right), i \in\right.$ $\{0, \ldots, M\}\}$.

The paper is organized as follows. Section 2 is devoted to the (formal) singular perturbation analysis of the current driven equations. In subsection 2.1 we define the reduced current driven and voltage driven equations, as well as the classical internal and boundary layer equations which are associated. We also recall some existing results on these equations. In subsection 2.2 we establish general properties of the (unique) solution of the reduced current driven equations $(\mathrm{RCD})_{I}$. We prove in particular that the set $\mathcal{E}$ of $I$ for which $(\mathrm{RCD})_{I}$ has a solution is not empty, is open, and that the solution of the reduced current driven equation is analytic on this set. We also prove that the points $I$ lying on the boundary of $\mathcal{E}$ correspond to saturation currents. In subsection 2.3 we introduce a non-classical internal layer problem in order to obtain a $\mathcal{C}^{1}$ approximation of the electrostatic potential. We prove general properties of the solutions of this problem, such as existence, uniqueness and exponential decay at infinity. Finally, in subsection 2.4 we define the modified asymptotic expansion, and prove that the asymptotic terms corresponding to concentrations of electrons and holes are uniformly (with respect to $x$ and $\varepsilon$ ) bounded away from zero. We state the main results of this paper in section 3: fundamental uniform estimate of the inverse of the linearized current driven equations (corollary 3.2 and theorem 3.1 ), asymptotic error estimates (theorem 3.3) and multiplicity results (theorem 3.4 and corollary 3.5). Section 4 is devoted to the proof of the main results. In Part II we give a constructive method for giving examples of data for which the electroneutral voltage driven equations have multiple solutions.

\section{Part I. The nonelectroneutral case}

The first part of this paper is devoted to the analysis of the singularly perturbed voltage driven equations. We prove in this part that if the associated reduced voltage driven equations have multiple solutions then the singularly perturbed equations also have multiple solutions, provided that $\varepsilon$ is sufficiently small.

\section{Singular Perturbation analysis of the CURREnt DRiven equations}

2.1. The formal asymptotic expansion. Let $\mathcal{P}_{M}=\left(a_{i}\right)_{0 \leq i \leq M+1}$ be a partition of $\bar{\Omega}$ which is independent of $\varepsilon$ and assume that $N \in W^{1,1}\left(\Omega, \overline{\mathcal{P}}_{M}\right)$. Since $\varepsilon$ is a small parameter which multiplies the highest derivative of equation (1.1), both systems $(\mathrm{CD})_{I, \varepsilon}$ and $(\mathrm{VD})_{V, \varepsilon}$ can be analyzed in the framework of singular perturbation theory (see e.g. [9]). We set

$$
S_{l}=\left\{i \in\{0, \ldots, M+1\}, n_{a_{i}}-p_{a_{i}} \neq N\left(a_{i}\right)\right\} .
$$

We recall that a formal asymptotic expansion which includes internal (resp. boundary) layer terms in a neighbourhood of the points $a_{i}, i \in\{1, \ldots, M\}$, such that $[N]_{a_{i}} \neq 0$ (resp. $i \in S_{l}$ ) can be associated to (VD) $)_{V, \varepsilon}$ by using standard techniques of singular perturbation theory (see e.g. [10]).

In this paper we are interested in a different approach which is based on a singular perturbation analysis of the current driven equations $(\mathrm{CD})_{I, \varepsilon}$ instead of $(\mathrm{VD})_{V, \varepsilon}$. In particular, it is important to remark that when one considers $(\mathrm{CD})_{I, \varepsilon}$ (resp. $\left.(\mathrm{VD})_{V, \varepsilon}\right), I$ is a given datum which is independent on $\varepsilon$ (resp. an unknown 
constant which depends on $\varepsilon$ ) whereas $V$ is an unknown constant which depends on $\varepsilon$ (resp. a given datum which is independent on $\varepsilon$ ).

Since the derivation of the formal asymptotic expansion associated to $(\mathrm{CD})_{I, \varepsilon}$ is similar (up to minor changes) to that of $(\mathrm{VD})_{V, \varepsilon}$, we just give briefly below the reduced and the layer current driven equations. We refer the reader to [10] for more details on the voltage driven case.

The reduced current driven equations are derived from $(\mathrm{CD})_{I, \varepsilon}$ by setting $\varepsilon=0$ in (1.1). Since at the points $a_{i}, i \in\{1, \ldots, M\}$, such that $[N]_{a_{i}} \neq 0$ (resp. $i \in$ $S_{l}$ ) the solutions of the reduced equations are discontinuous (resp. do not satisfy the boundary conditions (1.6)-(1.8)), these solutions cannot be expected to be a uniform approximation of the solution of the full problem $(\mathrm{CD})_{I, \varepsilon}$. In order to obtain an approximation which can be expected to be a uniform approximation, one must add correction (or layer) terms in a neighbourhood of the points $a_{i}$, $i \in\{1, \ldots, M\} \cup S_{l}$ (we will see later that if $i \in\{1, \ldots, M\}$ is such that $[N]_{a_{i}}=0$ then the corresponding zeroth order layer terms are equal to zero). Of course the layer terms must be small except in a neighbourhood of $a_{i}, i \in\{1, \ldots, M\} \cup S_{l}$. The zeroth order terms of the formal asymptotic expansion which is associated to $(\mathrm{CD})_{I, \varepsilon}$ have the form

$$
\left(\psi, n, p, J_{n}\right)(x)+\left(\hat{\psi}_{i}, \hat{n}_{i}, \hat{p}_{i}, \hat{J}_{n, i}\right)\left(\left(x-a_{i}\right) \varepsilon^{-1 / 2}\right),
$$

where $\left(\psi, n, p, J_{n}\right)$ is a solution of the reduced current driven equations and where $\left(\hat{\psi}_{i}, \hat{n}_{i}, \hat{p}_{i}, \hat{J}_{n, i}\right)$ are the layers terms which are defined on $\mathbb{R}$ (resp. $(-\infty, 0]$ or $[0,+\infty)$ ) if $i \in\{1, \ldots, M\}$ (resp. $i \in S_{l} \cap\{M+1\}$ or $i \in S_{l} \cap\{0\}$ ) and which decay exponentially at infinity. Of course these layer terms generally depend on the solutions of the reduced problem.

We now give the reduced and layer current driven equations. These equations are derived by inserting the expression $(2.2)$ in $(\mathrm{CD})_{I, \varepsilon}$ and by using the exponential decay of the layer terms at infinity. The interface and boundary conditions are derived by using standard matching techniques based on the regularity of the solution of $(\mathrm{CD})_{I, \varepsilon}$ and on the boundary conditions (1.6) and (1.8).

The reduced current driven equations are given by

$$
\begin{gathered}
n-p-N=0 \quad \text { in } \quad \Omega_{M}=\bigcup_{i=0}^{M}\left(a_{i}, a_{i+1}\right), \\
n^{\prime}=n \psi^{\prime}+J_{n} \quad \text { in } \quad \Omega_{M}, \\
p^{\prime}=-p \psi^{\prime}+J_{n}-I \quad \text { in } \Omega_{M}, \\
J_{n}^{\prime}=0 \quad \text { in } \quad \Omega_{M},
\end{gathered}
$$

subject to the boundary conditions

$$
\psi(-1)=\psi_{r,-1}, \quad n( \pm 1)=n_{r, \pm 1}, \quad p( \pm 1)=p_{r, \pm 1},
$$

and to the interface conditions

$$
[n \exp (-\psi)]_{a_{i}}=[p \exp (\psi)]_{a_{i}}=\left[J_{n}\right]_{a_{i}}=0 \quad \forall i \in\{1, \ldots, M\},
$$


where the boundary data are given by

$$
\begin{aligned}
& n_{r, x}=\frac{N(x)+\left(N^{2}(x)+4 \delta^{4}\right)^{1 / 2}}{2}, \quad p_{r, x}=\frac{-N(x)+\left(N^{2}(x)+4 \delta^{4}\right)^{1 / 2}}{2}, \\
& \quad \psi_{r,-1}=\log \left(\frac{n_{r,-1}}{n_{-1}}\right)+\psi_{-1} .
\end{aligned}
$$

We define the reduced voltage associated to $\left(\psi, n, p, J_{n}\right)$ by

$$
V=\log \left(\frac{n_{r, 1}}{n_{r,-1}}\right)+\psi_{r,-1}-\psi(1) .
$$

Of course $V$ depends on $I$. Since the reduced current driven system formed by (2.3)-(2.9) depends on $I$, we denote this system by $(\mathrm{RCD})_{I}$ from now on. The reduced voltage driven system consists of (2.3)-(2.10) and

$$
I^{\prime}=0 \quad \text { in } \quad \Omega_{M}, \quad[I]_{a_{i}}=0 \quad \forall i \in\{1, \ldots, M\},
$$

where (2.10) gives the boundary condition on $\psi$ at $x=1$. Since this system depends on $V$, it is denoted from now on by $(\mathrm{RVD})_{V}$.

We recall that the following result holds for the reduced voltage driven equations. For $N \in W^{1, \infty}\left(\Omega, \mathcal{P}_{M}\right)$ and for all $V \in \mathbb{R},(\operatorname{RVD})_{V}$ has at least one solution in $\left(W^{1, \infty}\left(\Omega, \mathcal{P}_{M}\right)\right)^{3} \times \mathbb{R}^{2}$ (see [10, theorem 4.4.1]). We also recall some recent results on $(\mathrm{RCD})_{I}$. We proved in [4, theorem 4.1] that if $N \in \mathcal{A}\left(\bar{\Omega}, \mathcal{P}_{M}\right)$ then for every $I$ in $\mathbb{R},(\mathrm{RCD})_{I}$ has at most one solution. On the other hand we also proved that if $N \in \mathcal{A}\left(\bar{\Omega}, \mathcal{P}_{M}\right)$ and satisfies

$$
N(-x)=-N(x), \quad N(x) \neq 0 \quad \forall x \in\left[a_{i}, a_{i+1}\right], \quad \forall x \in\{0, \ldots, M\},
$$

and

$$
\exists\left(i_{1}, i_{2}\right) \in(\{0, \ldots, M\})^{2} \quad \text { such that } \int_{a_{i_{1}}}^{1} N(t) d t<0<\int_{a_{i_{2}}}^{1} N(t) d t,
$$

then $(\mathrm{RCD})_{I}$ has a solution if and only if $I \in\left(I_{r, s}, I_{f, s}\right)$ where

$$
I_{r, s}=2 \delta^{4} \max _{i \in L_{r}}\left(\left(-\int_{a_{i}}^{1} N(t) d t\right)^{-1}\right)<0, \quad I_{f, s}=2 \delta^{4} \min _{i \in L_{f}}\left(\left(-\int_{a_{i}}^{1} N(t) d t\right)^{-1}\right)>0,
$$

with $L_{r}=\left\{i \in\{1, \ldots, M\}, \int_{a_{i}}^{1} N(t) d t>0\right\}$ and $L_{f}=\left\{i \in\{1, \ldots, M\}, \int_{a_{i}}^{1} N(t) d t\right.$ $<0\}$. Hence existence of solutions of $(\mathrm{RCD})_{I}$ for arbitrary $I$ cannot be expected to hold in general unless restrictive assumptions on the number of sign alterations of $N$ are made (see also $[15,17]$ ).

We define the reduced voltage function $\mathcal{V}$ as the map

$$
I \mapsto V,
$$

where $V$ is defined by $(2.10)$ ( $\psi$ being the electrostatic potentiel corresponding to a solution of $(\mathrm{RCD})_{I}$, when it exists). Moreover the reduced voltage current characteristic is the (possibly multi-valued) map

$$
V \mapsto I
$$

where $I$ is the total current coresponding to a solution of $(\mathrm{RVD})_{V}$.

We denote by $\left(\psi, n, p, J_{n}\right)$ a given solution of $(\mathrm{RCD})_{I}$ (if it exists). Then the internal layer terms $\hat{\psi}_{i}$ associated to $\left(\psi, n, p, J_{n}\right)$ and to $a_{i}, i \in\{1, \ldots, M\}$, are solutions of

$$
\frac{d^{2} \hat{\psi}_{i}}{d \tau^{2}}(\tau)=h_{i, \nu}\left(\hat{\psi}_{i}(\tau)\right) \quad \tau \in \mathbb{R}^{\nu}, \nu \in\{-,+\}
$$




$$
\begin{gathered}
\hat{\psi}_{i}\left(0^{+}\right)+\psi\left(a_{i}^{+}\right)=\hat{\psi}_{i}\left(0^{-}\right)+\psi\left(a_{i}^{-}\right), \\
\frac{d \hat{\psi}_{i}}{d \tau}\left(0^{+}\right)=\frac{d \hat{\psi}_{i}}{d \tau}\left(0^{-}\right), \\
\hat{\psi}_{i}(+\infty)=\hat{\psi}_{i}(-\infty)=0,
\end{gathered}
$$

where $h_{i, \nu}$ is the function defined by

$$
h_{i, \nu}(y)=n\left(a_{i}^{\nu}\right) \exp (y)-p\left(a_{i}^{\nu}\right) \exp (-y)-N\left(a_{i}^{\nu}\right), \quad y \in \mathbb{R},
$$

The boundary layer terms for $i \in S_{l}$ are solutions of

$$
\begin{gathered}
\frac{d^{2} \hat{\psi}_{i}}{d \tau^{2}}(\tau)=h_{i, \nu}\left(\hat{\psi}_{i}(\tau)\right), \quad \tau \in \mathbb{R}^{\nu}, \\
\hat{\psi}_{i}\left(0^{\nu}\right)=\log \left(\frac{n_{a_{i}}}{n_{r, a_{i}}}\right),
\end{gathered}
$$

where $\nu=+($ resp. -$)$ if $i=0$ (resp. $M+1)$. The internal and boundary layer terms $\hat{n}_{i}$ and $\hat{p}_{i}$ are given by the relations

$$
\begin{gathered}
\hat{n}_{i}(\tau)=n\left(a_{i}^{\nu}\right)\left(\exp \left(\hat{\psi}_{i}(\tau)\right)-1\right), \quad \tau \in \mathbb{R}^{\nu}, \nu \in \Upsilon, \\
\hat{p}_{i}(\tau)=p\left(a_{i}^{\nu}\right)\left(\exp \left(-\hat{\psi}_{i}(\tau)\right)-1\right), \quad \tau \in \mathbb{R}^{\nu}, \nu \in \Upsilon,
\end{gathered}
$$

where $\Upsilon=\{+,-\}$ if $i \in\{1, \ldots, M\}$, whereas $\Upsilon=\{+\}$ (resp. $\Upsilon=\{-\}$ ) if $i \in S_{l} \cap\{0\}$ (resp. $i \in S_{l} \cap\{M+1\}$ ). Moreover we have

$$
\hat{J}_{n, i}=0 \quad \forall i \in\{1, \ldots, M\} \cup S_{l}
$$

For a given solution $\left(\psi, n, p, J_{n}\right)$, if it exists (resp. $\left.\left(\psi, n, p, J_{n}, I\right)\right)$ of $(\mathrm{RCD})_{I}$ (resp. $\left.(\mathrm{RVD})_{V}\right)$ and for $i \in\{1, \ldots, M\}$, we denote by (ILP) $)_{I, i}(\text { resp. (ILP) })_{V, i}$ ) the internal layer current (resp. voltage) driven problem formed by (2.13)-(2.17). In the same way and for $i \in S_{l}$ we denote by (ILP) $I, i$ and (ILP) $)_{V, i}$ the boundary layer current (resp. voltage) driven problem formed by (2.17)-(2.19).

We now recall some results concerning the layer problem (ILP) $)_{V, i}$.

For a given solution $\left(\psi, n, p, J_{n}\right)\left(\operatorname{resp} .\left(\psi, n, p, J_{n}, I\right)\right)$ of $(\mathrm{RVD})_{V}\left(\operatorname{resp} .(\mathrm{RCD})_{I}\right.$, when it exists), existence and uniqueness of the solutions of (ILP) $V, i$ (and consequently of $\left.(\operatorname{ILP})_{I, i}\right)$ is proven in [10, theorem 4.5.1] by showing that (ILP) $V_{V, i}$ is equivalent to the problems

$$
\begin{gathered}
\frac{d^{2} \hat{\psi}_{i}}{d \tau^{2}}(\tau)=h_{i, \nu}\left(\hat{\psi}_{i}(\tau)\right), \quad \tau \in \mathbb{R}^{\nu}, \\
\hat{\psi}_{i}\left(0^{\nu}\right)=\alpha_{i}^{\nu}, \quad \hat{\psi}_{i}(\nu \infty)=0
\end{gathered}
$$

where $\nu \in\{+,-\}$ and $\alpha_{i}^{\nu}$ depend on $V$ and are given by

$$
\alpha_{i}^{\nu}=\frac{-[n+p]_{a_{i}}+N\left(a_{i}^{-\nu}\right)[\psi]_{a_{i}}}{[N]_{a_{i}}},
$$

and where $h_{i, \nu}$ are defined as in (2.17). Existence and uniqueness of the solutions of (2.23)-(2.24) for $\nu= \pm$ is then obtained by applying Fife's lemma ([7, lemma 2.1]) for solutions of problems of the form

$$
y^{\prime \prime}(t)=G(y(t)), \quad 0 \leq t<+\infty, y(0)=\alpha, y(+\infty)=0 .
$$


We also recall that the following properties hold:

$$
\alpha_{i}^{+}[N]_{a_{i}}<0, \quad \alpha_{i}^{-}[N]_{a_{i}}>0 \quad \forall i \in\{1, \ldots, M\} \text { such that }[N]_{a_{i}} \neq 0
$$

Moreover if $[N]_{a_{i}}<0$ (resp. $>0$ ) then $\hat{\psi}_{i}$ is decreasing (resp. increasing) on $(-\infty, 0)$ and $(0,+\infty)$.

It should be noted that if $i \in\{0, M+1\}-S_{l}$ then $n_{a_{i}}-p_{a_{i}}-N\left(a_{i}\right)=0$. Hence since $n_{a_{i}} p_{a_{i}}=\delta^{4}$ also holds, we deduce that $\left(n_{r, a_{i}}, p_{r, a_{i}}\right)=\left(n_{a_{i}}, p_{a_{i}}\right)$. In this case, the corresponding boundary layer terms $\hat{\psi}_{i}, \hat{n}_{i}$ and $\hat{p}_{i}$ vanish. It should also be noted that the boundary conditions for the reduced voltage driven problem always satisfy the electroneutrality condition

$$
n_{r, x}-p_{r, x}=N(x), \quad x= \pm 1
$$

2.2. On some qualitative properties of the reduced current driven equations. The purpose of this subsection is to give the possible shape in $\mathbb{R}^{2}$ of the reduced voltage function $\mathcal{V}$, which is defined by (2.11).

For given $N, \delta$ and $\mathcal{P}_{M}$ we need to introduce the following notation:

$$
\begin{gathered}
\mathcal{E}\left(N, \delta, \mathcal{P}_{M}\right)=\left\{I \in \mathbb{R},(R C D)_{I} \text { has a solution }\right\} \\
\mathcal{S}\left(V_{0}\right)=\left\{I \in \mathcal{E}\left(N, \delta, \mathcal{P}_{M}\right), \mathcal{V}(I)=V_{0}\right\} \\
\mathcal{S}_{n c}=\left\{I \in \mathcal{E}\left(N, \delta, \mathcal{P}_{M}\right), \mathcal{V}^{\prime}(I) \neq 0\right\}
\end{gathered}
$$

where $V_{0} \in \mathbb{R}$ and $\mathcal{V}$ is defined by (2.11). We also need the following spaces:

$$
\mathcal{C}_{\text {sat }, l}\left(N, \delta, \mathcal{P}_{M}\right)=\left\{I \in \mathcal{C}_{\text {sat }}\left(N, \delta, \mathcal{P}_{M}\right), \exists \eta>0,[I-\eta, I) \subset \mathcal{E}\left(N, \delta, \mathcal{P}_{M}\right)\right\},
$$

$$
\mathcal{C}_{\text {sat }, r}\left(N, \delta, \mathcal{P}_{M}\right)=\left\{I \in \mathcal{C}_{\text {sat }}\left(N, \delta, \mathcal{P}_{M}\right), \exists \eta>0,(I, I+\eta] \subset \mathcal{E}\left(N, \delta, \mathcal{P}_{M}\right)\right\} .
$$

We will also use the following definitions for the rest of this paper. An element of $\mathcal{C}_{\text {sat }}^{\nu}\left(N, \delta, \mathcal{P}_{M}\right)$ is called a positive (resp. negative) saturation current if $\nu=+$ (resp. - ). As will be seen in theorem 2.2, $|\mathcal{V}(I)|$ converges to $+\infty$ as $I$ converges to any element of $\mathcal{C}_{\text {sat }}\left(N, \delta, \mathcal{P}_{M}\right)$ in $\mathcal{E}\left(N, \delta, \mathcal{P}_{M}\right)$. This motivates the above definition. Moreover an element of $\mathcal{C}_{s a t, l}\left(N, \delta, \mathcal{P}_{M}\right)\left(\operatorname{resp} . \mathcal{C}_{s a t, r}\left(N, \delta, \mathcal{P}_{M}\right)\right)$ is called a left (resp. right) saturation current.

Of course an element of $\mathcal{C}_{\text {sat }}\left(N, \delta, \mathcal{P}_{M}\right)$ can be both a left and a right saturation current, or can be neither a left nor a right saturation current.

We can now state our results. It is easy to check that for $I=I_{e}=0,(\mathrm{RCD})_{I_{e}}$ has a unique solution $\left(\psi_{e}, n_{e}, p_{e}, J_{n, e}\right)$ which is given by

$$
\begin{aligned}
\psi_{e}(x) & =\psi_{r,-1}+\log \left(\frac{N(x)+\left(N^{2}(x)+4 \delta^{4}\right)^{1 / 2}}{2 n_{r,-1}}\right) \quad \forall x \in \bar{\Omega}, \\
n_{e}(x) & =n_{r,-1} \exp \left(-\psi_{r,-1}\right) \exp (\psi(x)), \\
p_{e}(x) & =p_{r,-1} \exp \left(\psi_{r,-1}\right) \exp (-\psi(x)) \quad \forall x \in \bar{\Omega}, \\
J_{n, e} & =0 .
\end{aligned}
$$


It can be easily checked that the linearized operator associated to $(\mathrm{RCD})_{I}$ at $\left(\psi_{e}, n_{e}, p_{e}, J_{n, e}\right)$ is boundedly invertible. Hence we deduce the following result

Proposition 2.1. Assume that $N \in W^{1,1}\left(\Omega, \mathcal{P}_{M}\right)$ and let $\delta>0$ be given. Then there exist $I_{-}(\delta)<0$ and $I_{+}(\delta)>0$ such that $(R C D)_{I}$ has a unique solution for every $I \in\left(I_{-}(\delta), I_{+}(\delta)\right)$.

We now give the possible shape of the reduced voltage function.

Theorem 2.2. Assume that $N \in W^{1, \infty}\left(\Omega, \mathcal{P}_{M}\right)$. Then for every $I \in \mathbb{R},(R C D)_{I}$ has at most one solution in $\left(W^{1, \infty}\left(\Omega, \mathcal{P}_{M}\right)\right)^{3} \times \mathbb{R}$. Moreover if $\bar{I} \in \mathcal{E}\left(N, \delta, \mathcal{P}_{M}\right)$, then the following properties hold:

(i): There exists an interval $\mathcal{O}\left(\bar{I}, N, \delta, \mathcal{P}_{M}\right)$, which is open, contains $\bar{I}$ and is such that $\mathcal{O}\left(\bar{I}, N, \delta, \mathcal{P}_{M}\right) \subset \mathcal{E}\left(N, \delta, \mathcal{P}_{M}\right)$.

(ii): $\mathcal{O}\left(\bar{I}, N, \delta, \mathcal{P}_{M}\right)$ is the unique maximal interval on which property (i) holds.

(iii): The solution $\left(\psi, n, p, J_{n}\right)(., I)$ of $(R C D)_{I}$ depends analytically on $I$ on $\mathcal{O}\left(\bar{I}, N, \delta, \mathcal{P}_{M}\right)$.

(iv): If $I_{0} \in \overline{\mathcal{E}\left(N, \delta, \mathcal{P}_{M}\right)}{ }^{\mathbb{R}}-\mathcal{E}\left(N, \delta, \mathcal{P}_{M}\right)$ then $I_{0} \neq 0$ and

$$
\lim _{I \rightarrow I_{0}, I \in \mathcal{E}\left(N, \delta, \mathcal{P}_{M}\right)} \mathcal{V}(I)=+\infty \operatorname{sign}\left(I_{0}\right) .
$$

(v): $\forall V_{0} \in \mathbb{R}, \mathcal{S}\left(V_{0}\right)$ is finite, where $\mathcal{S}\left(V_{0}\right)$ is defined by (2.28).

Proof. We already proved in [4] that if $N \in \mathcal{A}\left(\Omega, \mathcal{P}_{M}\right)$ then $(\mathrm{RCD})_{I}$ has at most one solution. Using the techniques of theorems 4.1 and 2.1 of [4] up to minor changes, it is easy to extend this uniqueness result to the more general case where $N \in W^{1, \infty}\left(\Omega, \mathcal{P}_{M}\right)$. We assume from now on that $N \in W^{1, \infty}\left(\Omega, \mathcal{P}_{M}\right)$. In order to prove (i) we proceed as follows. We introduce the following space equipped with its standard norm:

$$
E=W^{1, \infty}\left(\Omega, \mathcal{P}_{M}\right) \times\left(L^{\infty}\left(\Omega, \mathcal{P}_{M}\right)\right)^{3} \times \mathbb{R}^{3 M+5},
$$

and we set $U=\left(\psi, n, p, J_{n}\right)$. Then for each fixed $I \in \mathbb{R},(\mathrm{RCD})_{I}$ can be written in the abstract form $G(I, U)=0$, where $G$ is the map defined from $\mathbb{R} \times$ $\left(W^{1, \infty}\left(\Omega, \mathcal{P}_{M}\right)\right)^{4}$ to $E$ by $G(I, U)=(F(I, U), \Lambda(U))$ and where

$$
\begin{gathered}
F(I, U)_{/\left[a_{i}, a_{i+1}\right]} \\
=\left(n_{i}-p_{i}-N_{i}, n_{i}^{\prime}-\left(n_{i} \psi_{i}^{\prime}+J_{n, i}\right), p_{i}^{\prime}-\left(-p_{i} \psi_{i}^{\prime}+J_{n, i}-I\right), J_{n, i}^{\prime}\right), \\
i \in\{0, \ldots, M\}, \\
\Lambda(U)=\left([n \exp (-\psi)]_{a_{1}}, \ldots,[n \exp (-\psi)]_{a_{M}}, \ldots,[p \exp (\psi)]_{a_{1}}, \ldots,[p \exp (\psi)]_{a_{M},},\right. \\
{\left[J_{n}\right]_{a_{1}}, \ldots,\left[J_{n}\right]_{a_{M}}, \psi(-1)-\psi_{r,-1}, n(-1)-n_{r,-1},} \\
\left.n(1)-n_{r, 1}, p(-1)-p_{r,-1}, p(1)-p_{r, 1}\right),
\end{gathered}
$$

where $u_{i}=u_{/\left[a_{i}, a_{i+1}\right]}$. It is easy to check that $G$ is an analytic map from $\mathbb{R} \times$ $\left(W^{1, \infty}\left(\Omega, \mathcal{P}_{M}\right)\right)^{4}$ to $E$. Moreover, by using a proof similar to that of the uniqueness of solutions of $(\mathrm{RCD})_{I}$ we deduce that for every $\bar{I}$ such that $(\mathrm{RCD})_{\bar{I}}$ has a solution $U(\bar{I})$, the linearized operator $\partial_{U} G(\bar{I}, U(\bar{I}))$ is one-to-one. Since this operator is a Fredholm operator of index 0 , we can apply the analytic version of the implicit function theorem. This, together with the global uniqueness theorem for $(\mathrm{RCD})_{I}$, allows us to prove (i), (ii) and (iii). 
We prove (iv) as follows. We first remark that we have

$$
\forall I \in \mathcal{E}\left(N, \delta, \mathcal{P}_{M}\right), \mathcal{V}(I)>0(\text { resp. }<0,=0) \Leftrightarrow I>0(\text { resp. }<0,=0) .
$$

Let $I_{0} \in \overline{\mathcal{E}\left(N, \delta, \mathcal{P}_{M}\right)}{ }^{\overline{\mathbb{R}}}-\mathcal{E}\left(N, \delta, \mathcal{P}_{M}\right)$ be given. From proposition 2.1 we have $0 \in \mathcal{E}\left(N, \delta, \mathcal{P}_{M}\right)$. Hence $I_{0} \neq 0$. Assume first that $I_{0} \in(0,+\infty)$. We prove $(2.34)$ by contradiction. Hence assume that (2.34) does not hold. Therefore, thanks to (2.35), there exists a sequence $\left(I_{k}\right)_{k} \subset \mathcal{E}\left(N, \delta, \mathcal{P}_{M}\right)$ such that $\lim _{k \rightarrow+\infty} I_{k}=I_{0}$ and $\left(\mathcal{V}\left(I_{k}\right)\right)_{k}$ is bounded. We set $V_{k}=\mathcal{V}\left(I_{k}\right)$ and we denote by $\left(\psi_{k}, n_{k}, p_{k}, J_{n, k}\right)$ the solution of $(\mathrm{RCD})_{I_{k}}$. Then $\left(\psi_{k}, n_{k}, p_{k}, J_{n, k}, I_{k}\right)$ is a solution of (RVD) $V_{k}$. Therefore

$$
\left(n_{k} \exp \left(-\psi_{k}\right), p_{k} \exp \left(\psi_{k}\right)\right) \in\left(W^{1, \infty}(\Omega)\right)^{2}
$$

and $n_{k} \exp \left(-\psi_{k}\right), p_{k} \exp \left(\psi_{k}\right)$ are monotonic functions. Hence, since $\left(V_{k}\right)_{k}$ is bounded we deduce that the sequences $\left(n_{k} \exp \left(-\psi_{k}\right)\right)_{k}$ and $\left(p_{k} \exp \left(\psi_{k}\right)\right)_{k}$ are bounded in $L^{\infty}(\Omega)$ and are bounded away from zero independently on $k$. Using now the first equation of (RVD) $V_{k}$ we deduce that $\left(\psi_{k}\right)_{k}$ is bounded in $L^{\infty}(\Omega)$, so that $\left(n_{k}\right)_{k}$ and $\left(p_{k}\right)_{k}$ are also bounded in $L^{\infty}(\Omega)$ and are bounded away from zero independently on $k$. Moreover we have

$$
\left(\psi_{k /\left[a_{i}, a_{i+1}\right]}\right)^{\prime}=\frac{N_{i}^{\prime}-I_{k}}{n_{k}+p_{k}}, \quad i \in\{0, \ldots, M\} .
$$

Hence $\left(\psi_{k}\right)_{k}$ is bounded in $W^{1, \infty}\left(\Omega, \mathcal{P}_{M}\right)$. Using now (2.4)-(2.5) together with the inequalities $-\left|I_{k}\right| \leq J_{n, k} \leq\left|I_{k}\right|$, we deduce that $\left(n_{k}\right)_{k}$ and $\left(p_{k}\right)_{k}$ are bounded in $W^{1, \infty}\left(\Omega, \mathcal{P}_{M}\right)$. Since the imbedding of $W^{1, \infty}\left(\left[a_{i}, a_{i+1}\right]\right)$ in $\mathcal{C}^{0}\left(\left[a_{i}, a_{i+1}\right]\right)$ is compact, we deduce from Ascoli's theorem that there exists a subsequence of $\left(\psi_{k}, n_{k}, p_{k}, J_{n, k}\right)$ (still denoted by $\left.\left(\psi_{k}, n_{k}, p_{k}, J_{n, k}\right)\right)$ which converges in $\left(\mathcal{C}^{0}\left(\left[a_{i}, a_{i+1}\right]\right)\right)^{3} \times \mathbb{R}$ for all $i \in\{0, \ldots, M\}$ to $\left(\psi_{i}, n_{i}, p_{i}, J_{n, i}\right)$. We define $\left(\psi, n, p, J_{n}\right)$ on $\bar{\Omega}$ by its restrictions $\left(\psi_{i}, n_{i}, p_{i}, J_{n, i}\right)$ to the intervals $\left[a_{i}, a_{i+1}\right]$. Since (2.36) holds, we deduce that $\left(\psi_{k} /\left[a_{i}, a_{i+1}\right]\right)_{k}$ converges to $\psi_{/\left[a_{i}, a_{i+1}\right]}$ in $\mathcal{C}^{1}\left(\left[a_{i}, a_{i+1}\right]\right)$ for all $i \in\{0, \ldots, M\}$. In a similar way we deduce from (2.4)-(2.5) that $\left(n_{k} /\left[a_{i}, a_{i+1}\right]\right)_{k}$ and $\left(p_{k} /\left[a_{i}, a_{i+1}\right]\right)_{k}$ converges respectively to $n_{/\left[a_{i}, a_{i+1}\right]}$ and $p_{/\left[a_{i}, a_{i+1}\right]}$ in $\mathcal{C}^{1}\left(\left[a_{i}, a_{i+1}\right]\right)$. Moreover since $\left(\psi_{k}, n_{k}, p_{k}, J_{n, k}\right)$ satisfies $(2.7)-(2.9),\left(\psi, n, p, J_{n}\right)$ also satisfies (2.7)-(2.9). Hence $\left(\psi, n, p, J_{n}\right)$ is a solution of (RCD) $I_{0}$. This, together with the properties (i)-(ii), contradicts the hypothesis $I_{0} \notin \mathcal{E}\left(N, \delta, \mathcal{P}_{M}\right)$. Hence, thanks to (2.35), we deduce that $(2.34)$ holds. We prove in a similar way that the same result holds if $I_{0} \in(-\infty, 0)$.

Assume now that $I_{0}=+\infty$. Once again we prove (2.34) by contradiction. Assume that (2.34) does not hold; then thanks to (2.35), there exists a sequence $\left(I_{k}\right)_{k} \subset \mathcal{E}\left(N, \delta, \mathcal{P}_{M}\right)$ such that $\lim _{k \rightarrow+\infty} I_{k}=+\infty$ and $\left(\mathcal{V}\left(I_{k}\right)\right)_{k}$ is bounded. We set $V_{k}=\mathcal{V}\left(I_{k}\right)$ and we denote by $\left(\psi_{k}, n_{k}, p_{k}, J_{n, k}\right)$ the solution of $(\mathrm{RCD})_{I_{k}}$. Then $\left(\psi_{k}, n_{k}, p_{k}, J_{n, k}, I_{k}\right)$ is a solution of $(\mathrm{RVD})_{V_{k}}$. We deduce as for the case $I_{0} \in$ $(0,+\infty)$ that $\left(\psi_{k}\right)_{k},\left(n_{k}\right)_{k}$ and $\left(p_{k}\right)_{k}$ are bounded in $L^{\infty}(\Omega)$ and that moreover $\left(n_{k}\right)_{k}$ and $\left(p_{k}\right)_{k}$ are bounded away from zero independently of $k$. Integrating (2.36) from $a_{i}$ to $a_{i+1}$ for any $i \in\{0, \ldots, M\}$, we deduce that $\left(I_{k}\right)_{k}$ is bounded, which contradicts $\lim _{k \rightarrow+\infty} I_{k}=+\infty$. Hence (2.34) holds. The proof is similar if $I_{0}=$ $-\infty$.

Finally, property (v) follows easily from property (iv) and the analyticity of $V$ on $\mathcal{E}\left(N, \delta, \mathcal{P}_{M}\right)$. 
Thanks to the analyticity of $V$ on $\mathcal{E}\left(N, \delta, \mathcal{P}_{M}\right),(2.35)$, proposition 2.1 and properties (i)-(iv)-(v) of theorem 2.2 , we easily deduce the following result

Corollary 2.3. Assume that $N \in W^{1, \infty}\left(\Omega, \mathcal{P}_{M}\right), \delta>0$ and $\mathcal{P}_{M}$ are such that there exists $V_{0}>0$ (resp. <0) satisfying $\#\left(\mathcal{S}\left(V_{0}\right) \cap \mathcal{S}_{n c}\right)=k \geq 1$. Then, the following properties hold:

(i): $k$ is odd.

(ii): $\exists \eta \in\left(0, I^{\star}\right)$ such that $\left[I_{j}-\eta, I_{j}+\eta\right] \subset \mathcal{E}\left(N, \delta, \mathcal{P}_{M}\right)$ for all $j \in\{1, \ldots, k\}$, and

$$
\begin{aligned}
\mathcal{V}^{\prime}<0 & (\text { resp. }>0) \text { on }\left[I_{j}-\eta, I_{j}+\eta\right] \\
& \forall j \in\{2,4, \ldots, k-1\} \quad(\text { resp. } \quad j \in\{1,3, \ldots, k\}),
\end{aligned}
$$

where $I_{1}<\ldots<I_{k}$ denote the elements of $\mathcal{S}\left(V_{0}\right) \cap \mathcal{S}_{n c}$,

$$
I^{\star}=\min _{1 \leq j \leq k}\left(I_{j+1}-I_{j}\right)
$$

and \#S stands for the cardinal number of the finite subset $S$.

2.3. A non-classical internal layer problem. We remark that the function $g_{\varepsilon}(x)=\psi(x)+\hat{\psi}_{i}\left(\left(x-a_{i}\right) \varepsilon^{-1 / 2}\right)$ which corresponds to the first term of the asymptotic expansion (2.2) in a neighbourhood of $a_{i}$ is continuous in such a neighbourhood and is continuously differentiable on the left and the right hand side of $a_{i}$, but that in general $\left[g_{\varepsilon}^{\prime}\right]_{a_{i}} \neq 0$. Hence $g_{\varepsilon}$ cannot be a $\mathcal{C}^{1}$ approximation of $\psi_{\varepsilon}$. As will be seen in the sequel, it is convenient (in order to use theorem 3.1) to define a $\mathcal{C}^{1}$ approximation of $\psi_{\varepsilon}$. This is done by introducing a modified internal layer problem. Since we are also interested in the dependence of the reduced and layer equations on $I$, we specify the dependence on $I$ from now on. We set

$$
\tau_{i, \varepsilon}(x)=\frac{x-a_{i}}{\sqrt{\varepsilon}} .
$$

Moreover, for a given smooth function $(x, I) \mapsto f(x, I)$ we use the notation

$$
f^{\prime}(x, I)=\frac{\partial f}{\partial x}(x, I) .
$$

The modified internal layer current driven term associated to a given solution $\left(\psi, n, p, J_{n}\right)$ of $(\mathrm{RCD})_{I}$ for $I \in \mathcal{E}\left(N, \delta, \mathcal{P}_{M}\right)$ and to $a_{i}, i \in\{1, \ldots, M\}$, is denoted from now on by $\hat{\psi}_{i, \varepsilon}(., I)$. It is defined as the solution of the problem (2.13)-(2.14), (2.16), where $h_{i, \nu}$ is defined as in (2.17) and is subjected to the modified interface condition

$$
\left[\frac{\partial \hat{\psi}_{i, \varepsilon}(., I)}{\partial \tau}\right]_{0}=-\sqrt{\varepsilon}\left[\frac{\partial \psi(., I)}{\partial x}\right]_{a_{i}} .
$$

Thanks to the new interface condition $(2.38)$ the function $\psi(., I)+\hat{\psi}_{i, \varepsilon}\left(\tau_{i, \varepsilon}(), I.\right)$ is now continuously differentiable in a neighbourhood of $a_{i}$. We denote by (ILP) $)_{I, i, \varepsilon}$ the problem formed by equations (2.13)-(2.14), (2.16), (2.17) and (2.38). Of course the functions $h_{i, \nu}$ defined in (2.17) depend on $y$ and $I$.

The purpose of the next results is to show that (ILP) $)_{I, i, \varepsilon}$ has a unique solution for sufficiently small $\varepsilon$.

Theorem 2.4. Assume that $N \in W^{1, \infty}\left(\Omega, \mathcal{P}_{M}\right)$. Assume moreover that $I_{0} \in$ $\mathcal{E}\left(N, \delta, \mathcal{P}_{M}\right)$ and $i \in\{1, \ldots, M\}$ are such that $[N]_{a_{i}} \neq 0$ are given. Then for every compact subset $K$ of $\mathcal{O}\left(I_{0}, N, \delta, \mathcal{P}_{M}\right)$ there exist $\varepsilon_{0}=\varepsilon_{0}\left(i, K, I_{0}, N, \delta, \mathcal{P}_{M}\right)>0$ and 
$C_{1}=C_{1}\left(i, K, I_{0}, N, \delta, \mathcal{P}_{M}\right)>0$ such that for all $\varepsilon \in\left[0, \varepsilon_{0}\right]$ and all $I \in K$, the problem $(I L P)_{I, i, \varepsilon}$ has a unique solution $\hat{\psi}_{i, \varepsilon}(., I)$. Moreover this solution satisfies (2.13), $\hat{\psi}_{i, 0}(., I)=\hat{\psi}_{i}(., I)$ (where $\hat{\psi}_{i}(., I)$ denotes the solution of $\left.(I L P)_{I, i}\right)$ and

$$
\hat{\psi}_{i, \varepsilon}\left(0^{\nu}, I\right)=\alpha_{i, \varepsilon}^{\nu}(I), \quad \hat{\psi}_{i, \varepsilon}(\nu \infty, I)=0, \nu \in\{+,-\},
$$

where the functions $h_{i, \nu}(., I)$ are defined as in $(2.17),\left(\psi, n, p, J_{n}\right)(., I)$ being the unique solution of $(R C D)_{I}$, and where the numbers $\alpha_{i, \varepsilon}^{\nu}(I)$ are uniquely determined by the relations

$$
\begin{aligned}
G_{i}\left(\sqrt{\varepsilon}, I, \alpha_{i, \varepsilon}^{+}(I)\right) & =0, \quad \alpha_{i, \varepsilon}^{\nu}(I) \in V_{i}^{\nu}, \nu \in\{+,-\} \\
\left.\alpha_{i, \varepsilon}^{-}(I)\right) & =\alpha_{i, \varepsilon}^{+}(I)+[\psi(., I)]_{a_{i}}
\end{aligned}
$$

where the sets $V_{i}^{\nu}$ are compact neighbourhoods of $\left\{\alpha_{i, \varepsilon}^{\nu}(I), I \in K\right\}$ which are bounded away from zero. The functions $G_{i}(\lambda, I,$.$) are defined by$

$$
\begin{aligned}
& G_{i}(\lambda, I, x)=\left|[N]_{a_{i}}\right|\left(x-\alpha_{i}^{+}(I)-\frac{\lambda^{2} \beta_{i}^{2}(I)}{2[N]_{a_{i}}}\right) \\
& +\beta_{i}(I) \sqrt{2} \lambda\left(n\left(a_{i}^{+}, I\right)(\exp (x)-1)+p\left(a_{i}^{+}, I\right)(\exp (-x)-1)-N\left(a_{i}^{+}\right) x\right)^{1 / 2},
\end{aligned}
$$

where $\alpha_{i}^{\nu}(I), \nu \in\{+,-\}$ are defined as in (2.25) and where

$$
\beta_{i}(I)=-\left[\frac{\partial \psi(., I)}{\partial x}\right]_{a_{i}} .
$$

Moreover, the following properties hold:

(i): $\psi(., I)+\hat{\psi}_{i, \varepsilon}\left(\tau_{i, \varepsilon}(), I.\right)$ is a $\mathcal{C}^{1}$ function in a neighbourhood of $a_{i}$ for all $\varepsilon \in\left[0, \varepsilon_{0}\right]$ and all $I \in K$, where $\tau_{i, \varepsilon}$ is defined as in (2.37).

(ii): $(\tau, I) \mapsto \hat{\psi}_{i, \varepsilon}(\tau, I) \in \mathcal{C}^{\infty}((-\infty, 0], K) \cup \mathcal{C}^{\infty}([0,+\infty), K)$ for all $\varepsilon \in\left[0, \varepsilon_{0}\right]$.

(iii): The functions

$$
\tau \mapsto \frac{\partial^{p+q} \hat{\psi}_{i, \varepsilon}}{\partial \tau^{p} \partial I^{q}}(\tau, .)
$$

decay exponentially as $\tau \rightarrow \pm \infty$ with a decay rate that can be chosen independently of $(\varepsilon, I) \in\left[0, \varepsilon_{0}\right] \times K$ for all $(p, q) \in\{0,1,2\} \times\{0,1\}$.

(iv):

$$
\begin{aligned}
& \left|\frac{\partial^{p+q} \hat{\psi}_{i, \varepsilon}}{\partial \tau^{p} \partial I^{q}}\left(0^{\nu}, I\right)-\frac{\partial^{p+q} \hat{\psi}_{i}}{\partial \tau^{p} \partial I^{q}}\left(0^{\nu}, I\right)\right| \leq C_{1} \sqrt{\varepsilon} \\
& \quad \forall \varepsilon \in\left[0, \varepsilon_{0}\right], \quad \forall(p, q) \in\{0,1,2\} \times\{0,1\}, \nu \in\{+,-\}, \quad \forall I \in K .
\end{aligned}
$$

$(\mathrm{v})$ :

$$
\begin{aligned}
& \left|\frac{\partial^{q} \hat{\psi}_{i, \varepsilon}}{\partial I^{q}}(\tau, I)-\frac{\partial^{q} \hat{\psi}_{i}}{\partial I^{q}}(\tau, I)\right| \leq C_{1} \sqrt{\varepsilon} \\
& \quad \forall \varepsilon \in\left[0, \varepsilon_{0}\right], \quad \forall q \in\{0,1\}, \nu \in\{+,-\}, \quad \forall I \in K .
\end{aligned}
$$

Proof. Assume that $I_{0} \in \mathcal{E}\left(N, \delta, \mathcal{P}_{M}\right)$ and $i \in\{1, \ldots, M\}$ are given such that $[N]_{a_{i}} \neq 0$. Let $K$ be a subset of $\mathcal{O}\left(I_{0}, N, \delta, \mathcal{P}_{M}\right)$, and let $\varepsilon \geq 0$ be given. For $I \in K$ let us denote by $\left(\psi, n, p, J_{n}\right)(., I)$ the unique solution of $(\mathrm{RCD})_{I}$. Assume now that $\hat{\psi}_{i, \varepsilon}(., I)$ is a solution of (ILP) $)_{I, i, \varepsilon}$. Then we claim that

$$
\hat{\psi}_{i, \varepsilon}\left(0^{+}, I\right) \frac{\partial \hat{\psi}_{i, \varepsilon}}{\partial \tau}\left(0^{+}, I\right) \leq 0, \quad \hat{\psi}_{i, \varepsilon}\left(0^{-}, I\right) \frac{\partial \hat{\psi}_{i, \varepsilon}}{\partial \tau}\left(0^{-}, I\right) \geq 0 .
$$


We prove this claim as follows. Thanks to the maximum principle applied to equation (2.13), we deduce that if $\hat{\psi}_{i, \varepsilon}\left(0^{+}, I\right)>0($ resp. $<0,=0)$ then $\hat{\psi}_{i, \varepsilon}(\tau, I)>0$ (resp. $<0,=0$ ) for all $\tau \geq 0$. Using (2.13) once again, together with the relation $n\left(a_{i}^{+}, I\right)-p\left(a_{i}^{+}, I\right)-N\left(a_{i}^{+}\right)=0$, we deduce that if $\hat{\psi}_{i, \varepsilon}\left(0^{+}, I\right)>0($ resp. $<0)$ then $\hat{\psi}_{i, \varepsilon}(., I)$ is a strictly convex (resp. concave) function, so that $\frac{\partial \hat{\psi}_{i, \varepsilon}}{\partial \tau}(., I)$ is strictly increasing (resp. decreasing) on $\mathbb{R}^{+}$. Since this function vanishes at $\tau=+\infty$, we deduce that the first inequality of (2.45) holds. The proof of the second inequality is similar.

We multiply (2.13) by $\frac{\partial \hat{\psi}_{i, \varepsilon}}{\partial \tau}$ and integrate the resulting equation on $\mathbb{R}^{+}$and $\mathbb{R}^{-}$. This gives

$$
\begin{array}{r}
\left(\frac{\partial \hat{\psi}_{i, \varepsilon}}{\partial \tau}(\tau, I)\right)^{2}=2\left(n\left(a_{i}^{\nu}, I\right) \exp \left(\hat{\psi}_{i, \varepsilon}(\tau, I)\right)+p\left(a_{i}^{\nu}, I\right) \exp \left(-\hat{\psi}_{i, \varepsilon}(\tau, I)\right)\right. \\
\left.-N\left(a_{i}^{\nu}\right) \hat{\psi}_{i, \varepsilon}(\tau, I)+E_{i, \varepsilon}^{\nu}\right), \quad \nu \in\{+,-\}
\end{array}
$$

where $E_{i, \varepsilon}^{\nu}$ are unknown constants. Since $\hat{\psi}_{i, \varepsilon}( \pm \infty, I)=0$, we deduce from (2.46) that the limit

$$
\lim _{\tau \rightarrow \nu \infty} \frac{\partial \hat{\psi}_{i, \varepsilon}}{\partial \tau}(\tau, I)
$$

exists and is equal to zero for $\nu \in\{+,-\}$. This, together with (2.46), implies

$$
E_{i, \varepsilon}^{\nu}=-n\left(a_{i}^{\nu}, I\right)-p\left(a_{i}^{\nu}, I\right), \quad \nu \in\{+,-\} .
$$

We now set $\tau=0^{ \pm}$in (2.46). This, together with (2.47), yields

$$
\begin{array}{r}
\left(\frac{\partial \hat{\psi}_{i, \varepsilon}}{\partial \tau}\left(0^{\nu}, I\right)\right)^{2}=2\left(n\left(a_{i}^{\nu}, I\right)\left(\exp \left(\hat{\psi}_{i, \varepsilon}\left(0^{\nu}, I\right)\right)-1\right)\right. \\
+p\left(a_{i}^{\nu}, I\right)\left(\exp \left(-\hat{\psi}_{i, \varepsilon}\left(0^{\nu}, I\right)\right)-1\right) \\
\left.-N\left(a_{i}^{\nu}\right) \hat{\psi}_{i, \varepsilon}\left(0^{\nu}, I\right)\right), \quad \nu \in\{+,-\} .
\end{array}
$$

This, together with (2.8) and (2.14), leads to

$$
\left(\frac{\partial \hat{\psi}_{i, \varepsilon}}{\partial \tau}\left(0^{+}, I\right)\right)^{2}-\left(\frac{\partial \hat{\psi}_{i, \varepsilon}}{\partial \tau}\left(0^{-}, I\right)\right)^{2}=2[N]_{a_{i}}\left(\alpha_{i}^{+}(I)-\hat{\psi}_{i, \varepsilon}\left(0^{+}, I\right)\right)
$$

where $\alpha_{i}^{+}(I)$ is defined as in (2.25). On the other hand, thanks to (2.38) and (2.43) we have

$$
\left(\frac{\partial \hat{\psi}_{i, \varepsilon}}{\partial \tau}\left(0^{+}, I\right)\right)^{2}-\left(\frac{\partial \hat{\psi}_{i, \varepsilon}}{\partial \tau}\left(0^{-}, I\right)\right)^{2}=2\left(\sqrt{\varepsilon} \beta_{i}(I) \frac{\partial \hat{\psi}_{i, \varepsilon}}{\partial \tau}\left(0^{+}, I\right)-\frac{\varepsilon}{2} \beta_{i}^{2}(I)\right)
$$

From these last two relations we obtain

$$
\sqrt{\varepsilon} \beta_{i}(I) \frac{\partial \hat{\psi}_{i, \varepsilon}}{\partial \tau}\left(0^{+}, I\right)=[N]_{a_{i}}\left(\alpha_{i}^{+}(I)-\hat{\psi}_{i, \varepsilon}\left(0^{+}, I\right)\right)+\frac{\varepsilon}{2} \beta_{i}^{2}(I)
$$


We deduce in a similar way that

$$
\sqrt{\varepsilon} \beta_{i}(I) \frac{\partial \hat{\psi}_{i, \varepsilon}}{\partial \tau}\left(0^{-}, I\right)=[N]_{a_{i}}\left(\alpha_{i}^{-}(I)-\hat{\psi}_{i, \varepsilon}\left(0^{-}, I\right)\right)-\frac{\varepsilon}{2} \beta_{i}^{2}(I) .
$$

Thanks to (2.48) and (2.51) we have

$$
\begin{aligned}
& \sqrt{2 \varepsilon}\left|\frac{\beta_{i}(I)}{[N]_{a_{i}}}\right|\left[n\left(a_{i}^{+}, I\right)\left(\exp \left(\hat{\psi}_{i, \varepsilon}\left(0^{+}, I\right)\right)-1\right)+p\left(a_{i}^{+}, I\right)\left(\exp \left(-\hat{\psi}_{i, \varepsilon}\left(0^{+}, I\right)\right)-1\right)\right. \\
& \left.-N\left(a_{i}^{+}\right) \hat{\psi}_{i, \varepsilon}\left(0^{+}, I\right)\right]^{1 / 2}=\left|\alpha_{i}^{+}(I)-\hat{\psi}_{i, \varepsilon}\left(0^{+}, I\right)+\frac{\varepsilon}{2[N]_{a_{i}}} \beta_{i}^{2}(I)\right| .
\end{aligned}
$$

To find which sign must be chosen in (2.53) we must now use the inequalities (2.45). These inequalities, together with (2.51)-(2.52), yield

$$
\nu \beta_{i}(I)[N]_{a_{i}}\left(\alpha_{i}^{\nu}(I)-\hat{\psi}_{i, \varepsilon}\left(0^{\nu}, I\right)+\frac{\nu \varepsilon}{2[N]_{a_{i}}} \beta_{i}^{2}(I)\right) \hat{\psi}_{i, \varepsilon}\left(0^{\nu}, I\right) \leq 0, \quad \nu \in\{+,-\} .
$$

Several cases can now occur.

Case 1. Assume that $\beta_{i}(I)>0$ and $[N]_{a_{i}}>0$. This last inequality, together with (2.26), gives $\alpha_{i}^{-}(I)>0$. Hence for $\varepsilon$ sufficiently small (independently of $I \in K$ ) we have

$$
-\frac{\varepsilon}{2[N]_{a_{i}}} \beta_{i}^{2}(I)+\alpha_{i}^{-}(I)>0
$$

so that using (2.54) for $\nu=-$, we obtain

$$
\hat{\psi}_{i, \varepsilon}\left(0^{-}, I\right) \in\left[0,-\frac{\varepsilon}{2[N]_{a_{i}}} \beta_{i}^{2}(I)+\alpha_{i}^{-}(I)\right] .
$$

for $\varepsilon$ sufficiently small (independently of $I \in K$ ). Since $\hat{\psi}_{i, \varepsilon}$ and $\hat{\psi}_{i}$ satisfy (2.14) we have

$$
\alpha_{i}^{+}(I)=\alpha_{i}^{-}(I)-[\psi(., I)]_{a_{i}}, \quad \hat{\psi}_{i, \varepsilon}\left(0^{+}, I\right)=\hat{\psi}_{i, \varepsilon}\left(0^{-}, I\right)-[\psi(., I)]_{a_{i}} .
$$

Therefore (2.53) becomes in this first case

$$
\begin{array}{r}
\sqrt{2 \varepsilon} \frac{\beta_{i}(I)}{[N]_{a_{i}}}\left(n\left(a_{i}^{+}, I\right)\left(\exp \left(\hat{\psi}_{i, \varepsilon}\left(0^{+}, I\right)\right)-1\right)+p\left(a_{i}^{+}, I\right)\left(\exp \left(-\hat{\psi}_{i, \varepsilon}\left(0^{+}, I\right)\right)-1\right)\right. \\
\left.-N\left(a_{i}^{+}\right) \hat{\psi}_{i, \varepsilon}\left(0^{+}, I\right)\right)^{1 / 2}=\eta\left(\alpha_{i}^{+}(I)-\hat{\psi}_{i, \varepsilon}\left(0^{+}, I\right)+\frac{\varepsilon}{2[N]_{a_{i}}} \beta_{i}^{2}(I)\right) .
\end{array}
$$

with $\eta=1$.

Case 2. Assume that $\beta_{i}(I)<0$ and $[N]_{a_{i}}>0$. This last inequality, together with $(2.26)$ gives $\alpha_{i}^{+}(I)<0$, so that

$$
\frac{\varepsilon}{2[N]_{a_{i}}} \beta_{i}^{2}(I)+\alpha_{i}^{+}(I)<0,
$$

for $\varepsilon$ sufficiently small (independently of $I \in K$ ). Using (2.54) for $\nu=+$, we deduce that

$$
\hat{\psi}_{i, \varepsilon}\left(0^{+}, I\right) \in\left[\frac{\varepsilon}{2[N]_{a_{i}}} \beta_{i}^{2}(I)+\alpha_{i}^{+}(I), 0\right],
$$


for $\varepsilon$ sufficiently small (independently on $I \in K$ ). Hence (2.53) once again becomes (2.56) with $\eta=1$.

Case 3. Assume that $\beta_{i}(I)>0$ and $[N]_{a_{i}}<0$. This last inequality, together with (2.26), gives $\alpha_{i}^{+}(I)>0$, so that

$$
\frac{\varepsilon}{2[N]_{a_{i}}} \beta_{i}^{2}(I)+\alpha_{i}^{+}(I)>0
$$

for $\varepsilon$ sufficiently small (independently of $I \in K$ ). Using (2.54) for $\nu=+$, we deduce that (2.56) holds with $\eta=-1$.

Case 4. Assume that $\beta_{i}(I)<0$ and $[N]_{a_{i}}<0$. We deduce in the same way that (2.56) holds with $\eta=-1$.

Finally if $\beta_{i}(I)=0$ we prove easily that $\hat{\psi}_{i, \varepsilon}\left(0^{+}, I\right)=\alpha_{i}^{+}(I)$ and satisfies $G_{i}\left(\sqrt{\varepsilon}, I, \hat{\psi}_{i, \varepsilon}\left(0^{+}, I\right)\right)=0$.

Hence we deduce from these different cases that $\hat{\psi}_{i, \varepsilon}\left(0^{+}, I\right)$ satisfies

$$
G_{i}\left(\sqrt{\varepsilon}, I, \hat{\psi}_{i, \varepsilon}\left(0^{+}, I\right)\right)=0 \text { and }\left|\hat{\psi}_{i, \varepsilon}\left(0^{+}, I\right)\right| \leq C_{0}
$$

for all $\varepsilon \in\left[0, \varepsilon_{1}\right]$ and all $I \in K$. Here $G_{i}$ is defined as in (2.42) and $C_{0}, \varepsilon_{1}>0$ are constants which depend only on $i, K, N, \delta, I_{0}$ and $\mathcal{P}_{M}$. We now prove that $\hat{\psi}_{i, \varepsilon}\left(0^{+}, I\right)$ satisfies the second relation of $(2.40)$ as follows. If $[N]_{a_{i}}<0$ (resp. $>0)$, we know from $(2.26)$ that $\alpha_{i}^{+}(I)>0($ resp. $<0)$ and $\alpha_{i}^{-}(I)<0($ resp. $>0)$ for all $I \in K$. Moreover the maps $I \mapsto \alpha_{i}^{\nu}(I), \nu \in\{+,-\}$ are analytic on $K$. Hence the sets $V_{i}^{\nu}$ defined by

$$
V_{i}^{\nu}=\left[2^{-\nu \gamma_{i}} \min _{I \in K}\left(\alpha_{i}^{\nu}(I)\right), \quad 2^{\nu \gamma_{i}} \max _{I \in K}\left(\alpha_{i}^{\nu}(I)\right)\right],
$$

where $\gamma_{i}=1$ (resp. -1 ) if $[N]_{a_{i}}<0$ (resp. $>0$ ), are neighbourhoods of $\left\{\alpha_{i}^{\nu}(I), I \in\right.$ $K\}$ which are bounded away from zero.

Moreover, since we have

$$
\begin{aligned}
G_{i}(0, I, & \left.\hat{\psi}_{i, \varepsilon}\left(0^{+}, I\right)\right)-G_{i}\left(\sqrt{\varepsilon}, I, \hat{\psi}_{i, \varepsilon}\left(0^{+}, I\right)\right)=\left|[N]_{a_{i}}\right|\left(-\alpha_{i}^{+}(I)+\hat{\psi}_{i, \varepsilon}\left(0^{+}, I\right)\right) \\
= & \sqrt{\varepsilon}\left[\frac{\left|[N]_{a_{i}}\right|}{2[N]_{a_{i}}} \sqrt{\varepsilon} \beta_{i}^{2}(I)-\sqrt{2} \beta_{i}(I)\left(n\left(a_{i}^{+}, I\right)\left(\exp \left(\hat{\psi}_{i, \varepsilon}\left(0^{+}, I\right)\right)-1\right)\right.\right. \\
& \left.\left.+p\left(a_{i}^{+}, I\right)\left(\exp \left(-\hat{\psi}_{i, \varepsilon}\left(0^{+}, I\right)\right)-1\right)-N\left(a_{i}^{+}\right) \hat{\psi}_{i, \varepsilon}\left(0^{+}, I\right)\right)^{1 / 2}\right]
\end{aligned}
$$

and since $\left|\hat{\psi}_{i, \varepsilon}\left(0^{+}, I\right)\right| \leq C_{0}$, we deduce that

$$
\left|\hat{\psi}_{i, \varepsilon}\left(0^{+}, I\right)-\alpha_{i}^{+}(I)\right| \leq C_{2} \sqrt{\varepsilon} \quad \forall I \in K, \forall \varepsilon \in\left[0, \varepsilon_{1}\right],
$$

where $C_{2}$ and $\varepsilon_{1}>0$ depend only on $i, K, N, \delta, I_{0}$ and $\mathcal{P}_{M}$. Thanks to (2.55) we also have

$$
\left|\hat{\psi}_{i, \varepsilon}\left(0^{-}, I\right)-\alpha_{i}^{-}(I)\right| \leq C_{2} \sqrt{\varepsilon} \quad \forall I \in K, \forall \varepsilon \in\left[0, \varepsilon_{1}\right] .
$$

Hence there exists $\varepsilon_{0}>0$ depending only on $i, K, N, \delta, I_{0}$ and $\mathcal{P}_{M}$ such that $\hat{\psi}_{i, \varepsilon}\left(0^{\nu}, I\right) \in V_{i}^{\nu}$ for $\nu \in\{+,-\}$ and for all $I \in K$ and all $\varepsilon \in\left[0, \varepsilon_{0}\right]$. Moreover, since (2.14) holds, (2.41) is satisfied. Hence we have proved that if $\hat{\psi}_{i, \varepsilon}(., I)$ is a solution of (ILP) $)_{I, i, \varepsilon}$ then $\hat{\psi}_{i, \varepsilon}$ satisfies (2.13), (2.39) and (2.40)-(2.41).

Before proving that the converse is also true, we first prove that the equation $G_{i}(\sqrt{\varepsilon}, I, x)=0$ has a unique solution in $V_{i}^{+}$for all $\varepsilon \in\left[0, \varepsilon_{0}\right]$, where $\varepsilon_{0}>0$ depends only on $i, K, N, \delta, I_{0}$ and $\mathcal{P}_{M}$. We argue as follows. 
Since the set $V_{i}^{+}$is bounded away from zero we deduce that the function $(\lambda, I, x)$ $\mapsto G_{i}(\lambda, I, x) \in \mathcal{C}^{\infty}\left(\mathbb{R} \times K \times V_{i}^{+}\right)$and satisfies $\frac{\partial G_{i}}{\partial x}(\lambda, I, x) \geq C_{1}>0$ for all $\lambda \epsilon$ $\left[0, \sqrt{\varepsilon_{0}}\right]$, all $I \in K$ and all $x \in V_{i}^{+}$. Since $G_{i}\left(0, I, \alpha_{i}^{+}(I)\right)=0$ and $\alpha_{i}^{+}(I) \in V_{i}^{+}$for all $I \in K$, we deduce from the implicit function theorem together with the above inequality that the equation $G_{i}(\sqrt{\varepsilon}, I, x)=0$ has a unique solution $\hat{\psi}_{i, \varepsilon}\left(0^{+}, I\right)$ in $V_{i}^{+}$for all $\varepsilon \in\left[0, \varepsilon_{0}\right]$ and that the maps $I \mapsto \hat{\psi}_{i, \varepsilon}\left(0^{+}, I\right)$ are in $\mathcal{C}^{\infty}(K)$.

Assume now that $\hat{\psi}_{i, \varepsilon}(., I)$ is a piecewise $\mathcal{C}^{2}$ solution of (2.13), (2.39) and (2.40)(2.41). Hence $\hat{\psi}_{i, \varepsilon}(., I)$ satisfies (2.14). Thanks to (2.13)-(2.14) we deduce as before that $(2.45),(2.48)$ and $(2.49)$ hold. Moreover since (2.40) holds, we also deduce as before that (2.57) and (2.58) hold.

Assume now first that $[N]_{a_{i}}>0$. Then, thanks to (2.26), we have $\alpha_{i}^{+}(I)<0$ and $\alpha_{i}^{-}(I)>0$. Since (2.57) and (2.58) hold, we deduce that there exist $C_{3}>0$ and $\varepsilon_{0}>0$ such that

$$
\hat{\psi}_{i, \varepsilon}\left(0^{+}, I\right)<-C_{3}, \quad \hat{\psi}_{i, \varepsilon}\left(0^{-}, I\right)>C_{3} \quad \forall I \in K .
$$

Thanks to $(2.45)$ we obtain $\frac{\partial \hat{\psi}_{i, \varepsilon}}{\partial \tau}\left(0^{\nu}, I\right) \geq 0$ for $\nu \in\{+,-\}$. This, together with (2.48) and (2.40), yields

$$
[N]_{a_{i}}\left(\hat{\psi}_{i, \varepsilon}\left(0^{+}, I\right)-\alpha_{i}^{+}(I)\right)-\frac{\varepsilon \beta_{i}^{2}(I)}{2}+\beta_{i}(I) \sqrt{\varepsilon} \frac{\partial \hat{\psi}_{i, \varepsilon}}{\partial \tau}\left(0^{+}, I\right)=0 .
$$

Using (2.49) in this last equality, we obtain

$$
\left(\frac{\partial \hat{\psi}_{i, \varepsilon}}{\partial \tau}\left(0^{+}, I\right)-\beta_{i}(I) \sqrt{\varepsilon}\right)^{2}=\left(\frac{\partial \hat{\psi}_{i, \varepsilon}}{\partial \tau}\left(0^{-}, I\right)\right)^{2} .
$$

Using (2.59) in (2.48) together with (2.45), we deduce that

$$
\frac{\partial \hat{\psi}_{i, \varepsilon}}{\partial \tau}\left(0^{+}, I\right)-\beta_{i}(I) \sqrt{\varepsilon}>0
$$

for sufficiently small $\varepsilon$. In a similar way we prove that

$$
\frac{\partial \hat{\psi}_{i, \varepsilon}}{\partial \tau}\left(0^{-}, I\right)>0
$$

for sufficiently small $\varepsilon$. Hence (2.38) holds. The proof of (2.38) is similar in the case where $[N]_{a_{i}}<0$.

Thus we have proved that the problem (ILP) $)_{I, i, \varepsilon}$ is equivalent to (2.13), (2.39) and (2.40)-(2.41) for $\varepsilon$ sufficiently small. Using Fife's lemma ([7, lemma 2.1]) for the problems formed by $(2.13)$ and $(2.39)$ we now deduce that (ILP) $)_{I, i, \varepsilon}$ has a unique solution $\hat{\psi}_{i, \varepsilon}(., I)$ fo all $(\varepsilon, I) \in\left[0, \varepsilon_{0}\right] \times K$, which together with its derivatives with respect to $\tau$ decays exponentially as $\tau$ goes to $\pm \infty$. Since the coefficients in (2.13) and the boundary data in (2.39) depend in a $\mathcal{C}^{\infty}$ way on $I$, we deduce that (ii) holds. Moreover thanks to (2.57) and (2.58), we obtain (2.44) for $p=q=0$ and $\nu \in\{+,-\}$. Using (2.44) for $p=q=0$ together with (2.45), we easily deduce that (2.44) also holds for $p=1, q=0$ and $\nu \in\{+,-\}$. Using (2.13) together with (2.44) for $(p, q) \in\{0,1\} \times\{0\}$, we obtain (2.44) for $p=2, q=0$ and $\nu \in\{+,-\}$. Thanks 
to $(2.40)$ we have

$$
\frac{\partial G_{i}}{\partial I}\left(\sqrt{\varepsilon}, I, \hat{\psi}_{i, \varepsilon}\left(0^{+}, I\right)\right)+\frac{\partial G_{i}}{\partial x}\left(\sqrt{\varepsilon}, I, \hat{\psi}_{i, \varepsilon}\left(0^{+}, I\right)\right) \frac{\partial \hat{\psi}_{i, \varepsilon}}{\partial I}\left(0^{+}, I\right)=0 .
$$

Thanks to the definition (2.42) of $G_{i}$ we obtain

$$
\frac{\partial G_{i}}{\partial I}(\sqrt{\varepsilon}, I, x)=\frac{\partial G_{i}}{\partial I}(0, I, x)-\frac{\left|[N]_{a_{i}}\right|}{[N]_{a_{i}}} \varepsilon \beta_{i}(I) \frac{d \beta_{i}}{d I}(I)+\sqrt{2 \varepsilon} T_{i}(x, I),
$$

and

$$
\frac{\partial G_{i}}{\partial x}(\sqrt{\varepsilon}, I, x)=\frac{\partial G_{i}}{\partial x}(0, I, x)+\sqrt{2 \varepsilon} \beta_{i}(I) K_{i}(x, I),
$$

where $T_{i}$ and $K_{i}$ are smooth functions on $V_{i}^{\nu} \times K$ which are independent of $\varepsilon$. Using these last two expressions in (2.60), we deduce that (2.44) holds for $p=0, q=$ $1, \nu=+$. This, together with (2.41) yields (2.44) for $p=0, q=1, \nu=-$. The proof of (2.44) for the cases $p \in\{1,2\}, q=1$ and $\nu \in\{+,-\}$ is similar.

Thanks to (2.44) and following Fife's proofs ([7, lemma 2.1, 2.2]), it is easy to check that (iii) holds. We now prove (v) as follows. The function $\hat{\psi}_{i, \varepsilon}(., I)-\hat{\psi}_{i}(., I)$ satisfies the equation

$$
\begin{gathered}
\frac{\partial^{2}\left(\hat{\psi}_{i, \varepsilon}-\hat{\psi}_{i}\right)}{\partial \tau^{2}}(\tau, I)=h_{i, \nu}\left(\hat{\psi}_{i, \varepsilon}(\tau, I), I\right)-h_{i, \nu}\left(\hat{\psi}_{i}(\tau, I), I\right), \quad \tau \in \mathbb{R}^{\nu}, \nu \in\{+,-\}, \\
\hat{\psi}_{i, \varepsilon}\left(0^{\nu}, I\right)-\hat{\psi}_{i}\left(0^{\nu}, I\right)=\alpha_{i, \varepsilon}^{\nu}-\alpha_{i,}^{\nu}, \quad \hat{\psi}_{i, \varepsilon}(\nu \infty, I)-\hat{\psi}_{i}(\nu \infty, I)=0 .
\end{gathered}
$$

Since the functions $x \mapsto h_{i, \nu}(x, I)$ are strictly increasing on $\mathbb{R}$, we deduce from the maximum principle applied to the above equation that

$$
\min \left(0, \alpha_{i, \varepsilon}^{\nu}-\alpha_{i}^{\nu}\right) \leq\left(\hat{\psi}_{i, \varepsilon}-\hat{\psi}_{i}\right)(\tau, I) \leq \max \left(0, \alpha_{i, \varepsilon}^{\nu}-\alpha_{i}^{\nu}\right), \quad \forall \tau \in \mathbb{R}^{\nu}, \nu \in\{+,-\} .
$$

Using (2.44) with $p=q=0$ we deduce that (iv) holds for $q=0$. Differentiating (2.13) with respect to $I$ we obtain the equation

$$
\begin{array}{r}
\frac{\partial^{3}}{\partial \tau^{2} \partial I}\left(\hat{\psi}_{i, \varepsilon}-\hat{\psi}_{i}\right)(\tau, I)=\left(n\left(a_{i}^{\nu}, I\right) \exp \left(\hat{\psi}_{i, \varepsilon}(\tau, I)\right)+p\left(a_{i}^{\nu}, I\right) \exp \left(-\hat{\psi}_{i, \varepsilon}(\tau, I)\right)\right) \\
\times \frac{\partial}{\partial I}\left(\hat{\psi}_{i, \varepsilon}-\hat{\psi}_{i}\right)(\tau, I)+R_{i, \varepsilon}^{\nu}(\tau, I) \quad \tau \in \mathbb{R}^{\nu}, I \in K, \nu \in\{+,-\},
\end{array}
$$

where $\left\|R_{i, \varepsilon}^{\nu}\right\|_{0, \infty, \mathbb{R}^{\nu} \times K} \leq C \sqrt{\varepsilon}$. Using once again the maximum principle on the above equation together with (2.44) for $p=0, q=1$, we deduce that (v) holds for $q=1$. This concludes the proof of the theorem.

The above construction of a $\mathcal{C}^{1}$ approximation of $\psi_{\varepsilon}$ holds for the points $a_{i}$, $i \in\{1, \ldots, M\}$, such that $[N]_{a_{i}} \neq 0$. It fails when $[N]_{a_{i}}=0$. Hence we have to proceed differently in this latter (indeed simpler) case. This is done in the following proposition (whose proof is left to the reader).

Proposition 2.5. Assume that $N \in W^{1, \infty}\left(\Omega, \mathcal{P}_{M}\right)$. Assume moreover that $I_{0} \in$ $\mathcal{E}\left(N, \delta, \mathcal{P}_{M}\right)$ and $i \in\{1, \ldots, M\}$ such that $[N]_{a_{i}}=0$ are given. We set for every compact subset $K$ of $\mathcal{O}\left(I_{0}, N, \delta, \mathcal{P}_{M}\right)$, all $I \in K$ and all $\tau \in \mathbb{R}$

$$
\begin{aligned}
& \hat{\psi}_{i, \varepsilon}(\tau, I)=\frac{-\beta_{i}(I)}{2 \sqrt{\left|\left[N^{\prime}\right]_{a_{i}}\right|}} \sqrt{\varepsilon} \exp \left(-\sqrt{\left|\left[N^{\prime}\right]_{a_{i}}\right|}|\tau|\right) \quad(\text { resp. }=0) \\
& \text { if } \left.\left[N^{\prime}\right]_{a_{i}} \neq 0 \quad \text { (resp. if }\left[N^{\prime}\right]_{a_{i}}=0\right),
\end{aligned}
$$


where $\beta_{i}(I)$ is defined as in (2.43). Then the function $\hat{\psi}_{i, \varepsilon}$ defined by the above relation satisfies the properties (i)-(v) of theorem 2.4.

2.4. Modified asymptotic expansion. We are now able to introduce the first order term of the modified asymptotic solution associated to the solution of $(\mathrm{CD})_{I, \varepsilon}$ for $I \in \mathcal{E}\left(N, \delta, \mathcal{P}_{M}\right)$. We proceed as follows.

For a given partition $\mathcal{P}_{M}=\left(a_{i}\right)_{0 \leq i \leq M+1}$ of $\bar{\Omega}$ we choose $\Delta$ such that

$$
0<\Delta<3^{-1} \min _{j \neq i, 0 \leq i, j \leq M+1}\left(\left|a_{i}-a_{j}\right|\right) .
$$

Moreover for $i \in\{1, \ldots, M\} \cup S_{l}$ and $\Delta$ as above, we define truncating functions $M_{i, \Delta} \in \mathcal{C}^{\infty}(\bar{\Omega})$ by

$$
\begin{aligned}
& M_{i, \Delta}(x)=1 \quad \text { for } \quad\left|x-a_{i}\right| \leq \Delta, \quad M_{i, \Delta}(x)=0 \quad \text { for } \quad\left|x-a_{i}\right| \geq 2 \Delta \\
& 0 \leq M_{i, \Delta}(x) \leq 1 \quad \text { for } \quad \Delta \leq\left|x-a_{i}\right| \leq 2 \Delta .
\end{aligned}
$$

We remark that (2.62) yields

$$
M_{i, \Delta}(x)=0 \quad \text { for } \quad\left|x-a_{j}\right| \leq \Delta, \quad x \in \bar{\Omega}, \quad \forall j \neq i .
$$

We set

$$
P=\left\{i \in\{1, \ldots, M\},[N]_{a_{i}}=0\right\}, \quad Q=\left\{i \in\{1, \ldots, M\},[N]_{a_{i}} \neq 0\right\},
$$

and for all $I \in \mathcal{E}\left(N, \delta, \mathcal{P}_{M}\right)$

$$
\begin{gathered}
\tilde{\psi}_{\varepsilon}(x, I)=\psi(x, I)+\sum_{i \in P \cup Q \cup S_{l}} \hat{\psi}_{i, \varepsilon}\left(\tau_{i, \varepsilon}(x), I\right) M_{i, \Delta}(x), \\
\tilde{n}_{\varepsilon}(x, I)=n(x, I)+\sum_{i \in Q \cup S_{l}} n\left(a_{i}^{\nu}, I\right)\left(\exp \left(\hat{\psi}_{i, \varepsilon}\left(\tau_{i, \varepsilon}(x), I\right)\right)-1\right) M_{i, \Delta}(x),
\end{gathered}
$$

$$
\tilde{p}_{\varepsilon}(x, I)=p(x, I)+\sum_{i \in Q \cup S_{l}} p\left(a_{i}^{\nu}, I\right)\left(\exp \left(-\hat{\psi}_{i, \varepsilon}\left(\tau_{i, \varepsilon}(x), I\right)\right)-1\right) M_{i, \Delta}(x),
$$

where $\nu=\nu(x)=+($ resp. -$)$ if $x \geq a_{i}($ resp $\leq), \tau_{i, \varepsilon}$ is defined by $(2.37)$ and $\hat{\psi}_{i, \varepsilon}$ is defined as in theorem 2.4 (resp. proposition 2.5) if $i \in Q$ (resp. P). Moreover we have also set $\hat{\psi}_{i, \varepsilon}=\hat{\psi}_{i}$ for $i \in S_{l}$, where $\hat{\psi}_{i}$ is the solution of (ILP) $I, i$.

The following result will be useful below.

Lemma 2.6. Assume that $N \in W^{1, \infty}\left(\Omega, \mathcal{P}_{M}\right)$. Then for every $I \in \mathcal{E}\left(N, \delta, \mathcal{P}_{M}\right)$ and every compact subset $K$ of $\mathcal{O}\left(I, N, \delta, \mathcal{P}_{M}\right)$ there exists $\varepsilon_{1}=\varepsilon_{1}\left(K, I_{0}, N, \delta, \mathcal{P}_{M}\right)$ $>0$ such that for every $\varepsilon \in\left(0, \varepsilon_{1}\right]$

$$
\left(\tilde{\psi}_{\varepsilon}, \tilde{n}_{\varepsilon}, \tilde{p}_{\varepsilon}\right) \in \mathcal{C}^{1}(\bar{\Omega} \times K) \times\left(\mathcal{C}^{0}(\bar{\Omega} \times K)\right)^{2} .
$$

Moreover let $I_{0} \in \mathcal{E}\left(N, \delta, \mathcal{P}_{M}\right)$ be given. Then for every compact subset $K$ of $\mathcal{O}\left(I_{0}, N, \delta, \mathcal{P}_{M}\right)$ there exist $\Delta_{0}=\Delta_{0}\left(K, I_{0}, N, \delta, \mathcal{P}_{M}\right)>0$ satisfying (2.62), and $r_{-}=r_{-}\left(K, I_{0}, N, \delta, \mathcal{P}_{M}\right)>0$ such that

$$
\tilde{n}_{\varepsilon}(x, I) \geq r_{-}, \quad \tilde{p}_{\varepsilon}(x, I) \geq r_{-} \quad \forall x \in \bar{\Omega}, \forall I \in K, \forall \varepsilon \in\left(0, \varepsilon_{1}\right], \forall \Delta \in\left(0, \Delta_{0}\right] .
$$


Proof. From our choice of the functions $\hat{\psi}_{i, \varepsilon}$ it is clear that

$$
\left(\tilde{\psi}_{\varepsilon}, \tilde{n}_{\varepsilon}, \tilde{p}_{\varepsilon}\right) \in \mathcal{C}^{1}(\bar{\Omega} \times K) \times\left(\mathcal{C}^{0}(\bar{\Omega} \times K)\right)^{2}
$$

for $\varepsilon$ sufficiently small.

Assume now that $I_{0} \in \mathcal{E}\left(N, \delta, \mathcal{P}_{M}\right)$ is given and let $K$ be any compact subset of $\mathcal{O}\left(I_{0}, N, \delta, \mathcal{P}_{M}\right)$. Using the exponential decay (with a decay rate chosen independently of $\varepsilon, I \in K$ and $i$ ) of $\hat{\psi}_{i, \varepsilon}$ at $\tau= \pm \infty$ and the fact that there exists $\eta>0$ such that $n(x, I) \geq \eta$ and $p(x, I) \geq \eta$ for all $x \in \bar{\Omega}$ and all $I$ in $K$ we deduce easily that for every $\Delta$ satisfying (2.62) we have

$$
\tilde{n}_{\varepsilon}(x, I) \geq \frac{\eta}{2}, \quad \tilde{p}_{\varepsilon}(x, I) \geq \frac{\eta}{2} \quad \forall x \in \bar{\Omega}-\left(\bigcup_{i \in Q \cup S_{l}}\left[a_{i}-\Delta, a_{i}+\Delta\right]\right),
$$

for $\varepsilon$ sufficiently small and independent of $x$ and $I$.

Assume now that

$$
x \in \bar{\Omega} \cap\left(\bigcup_{i \in Q \cup S_{l}}\left[a_{i}-\Delta, a_{i}+\Delta\right]\right) .
$$

Hence there exists $j \in Q \cup S_{l}$ such that $\left|x-a_{j}\right| \leq \Delta$. Thanks to (2.64) we have

$$
\tilde{n}_{\varepsilon}(x, I)=n(x, I)+n\left(a_{j}^{\nu}, I\right)\left(\exp \left(\hat{\psi}_{j}\left(\tau_{j, \varepsilon}(x), I\right)-1\right)+g_{j, \varepsilon}^{\nu}\left(\tau_{j, \varepsilon}(x), I\right),\right.
$$

where $g_{j, \varepsilon}^{\nu}(y, I)=0$ if $j \in S_{l}$ and $g_{j, \varepsilon}^{\nu}(y, I)=n\left(a_{j}^{\nu}, I\right)\left(\exp \left(\hat{\psi}_{j, \varepsilon}(y, I)\right)-\exp \left(\hat{\psi}_{j}(y, I)\right)\right)$ if $j \in Q$. Using the property (v) of theorem 2.4 and proposition 2.5 we have for $\varepsilon$ sufficiently small

$$
\begin{aligned}
& \left|g_{j, \varepsilon}^{\nu}\left(\tau_{j, \varepsilon}(x), I\right)\right| \leq C \sqrt{\varepsilon} \quad \forall j \in Q \cup S_{l}, \\
& \left.\forall x \in \bar{\Omega} \cap\left[a_{j}-\Delta, a_{j}+\Delta\right]\right), \forall I \in K .
\end{aligned}
$$

On the other hand, the maximum principle applied to equation (2.13) yields

$$
\min \left(\hat{\psi}_{j}\left(0^{\nu}, I\right), 0\right) \leq \hat{\psi}_{j}(\tau, I) \leq \max \left(\hat{\psi}_{j}\left(0^{\nu}, I\right), 0\right), \forall \tau \in R^{\nu} .
$$

This, together with (2.71) leads to

$$
\begin{aligned}
& \tilde{n}_{\varepsilon}(x, I) \geq n(x, I)-n\left(a_{j}^{\nu}, I\right)+\eta_{1}-C \sqrt{\varepsilon} \quad \forall j \in Q \cup S_{l}, \\
& \forall x \in \bar{\Omega} \cap\left[a_{j}-\Delta, a_{j}+\Delta\right], \quad \forall I \in K,
\end{aligned}
$$

where

$$
\eta_{1}=\min _{I \in K, j \in Q \cup S_{l}, \nu}\left(n\left(a_{j}^{\nu}, I\right) \exp \left(\min \left(\hat{\psi}_{j}\left(0^{\nu}, I\right), 0\right)\right)>0 .\right.
$$

Using the left and right continuity of $n(., I)$ at $x=a_{j}$, and since $n$ is smooth with respect to $I$ on the compact $K$, we deduce that there exist $\Delta_{0}=\Delta_{0}\left(K, I_{0}, N, \delta, \mathcal{P}_{M}\right)>$ 0 and $r_{-}=r_{-}\left(K, I_{0}, N, \delta, \mathcal{P}_{M}\right)>0$ such that

$$
\begin{aligned}
& n(x, I)-n\left(a_{j}^{\nu}, I\right)+\eta_{1} \geq r_{-} \quad \forall j \in Q \cup S_{l}, \forall x \in \bar{\Omega} \cap\left[a_{j}-\Delta, a_{j}+\Delta\right], \\
& \forall I \in K, \quad \forall \Delta \in\left(0, \Delta_{0}\right] .
\end{aligned}
$$

Using this last inequality in (2.72), we obtain the first inequality of (2.69). The proof of the second inequality is similar. 


\section{MAin RESUlts}

We need the following spaces for the sequel:

$$
X=W^{3,1}\left(\Omega, \mathcal{P}_{M}\right) \times\left(W^{1,1}(\Omega)\right)^{3}, \quad Y=W^{1,1}\left(\Omega, \mathcal{P}_{M}\right) \times\left(L^{1}(\Omega)\right)^{3} \times \mathbb{R}^{5} .
$$

These spaces are equipped with the norms respectively defined by

$$
\begin{gathered}
\|U\|_{X}=\left\|u_{1}\right\|_{3,1, \Omega, \mathcal{P}_{M}}+\sum_{i=2}^{i=4}\left\|u_{i}\right\|_{1,1, \Omega}, \quad U=\left(u_{i}\right)_{1 \leq i \leq 4} \in X, \\
\|R\|_{Y}=\left\|R_{1}\right\|_{1,1, \Omega, \mathcal{P}_{M}}+\sum_{i=2}^{i=4}\left\|R_{i}\right\|_{0,1, \Omega}+\sum_{i=5}^{i=9}\left|R_{i}\right|, \quad R=\left(R_{i}\right)_{1 \leq i \leq 9} \in Y .
\end{gathered}
$$

We consider given functions $a, b, c$ satisfying

$$
(a, b, c) \in\left(W^{1,1}(\Omega)\right)^{3},
$$

and

$$
\exists r_{-}>0 \quad \text { such that } \quad r_{-} \leq \min (b(x), c(x)) \quad \forall x \in \bar{\Omega} .
$$

We associate to $(a, b, c)$ the continuous operator $A_{(a, b, c)}^{\varepsilon}$ acting from $X$ to $Y$ and defined by

$$
\begin{aligned}
A_{(a, b, c)}^{\varepsilon} U= & \left(\varepsilon u_{1}^{\prime \prime}-\left(u_{2}-u_{3}\right), u_{2}^{\prime}-\left(a^{\prime} u_{2}+b u_{1}^{\prime}+u_{4}\right),\right. \\
& \left.u_{3}^{\prime}+\left(a^{\prime} u_{3}+c u_{1}^{\prime}-u_{4}\right), u_{4}^{\prime}, u_{1}(-1), u_{2}(-1), u_{2}(1), u_{3}(-1), u_{3}(1)\right),
\end{aligned}
$$

where $U=\left(u_{i}\right)_{1 \leq i \leq 4} \in X$. We remark that in the particular case where $\left(a, b, c, J_{n}\right)$ is a solution of $(\overline{\mathrm{CD}})_{I, \varepsilon}$, then $A_{(a, b, c)}^{\varepsilon}$ is the linearized operator at $\left(a, b, c, J_{n}\right)$ which is associated to $(\mathrm{CD})_{I, \varepsilon}$. That is the reason why we call $A_{(a, b, c)}^{\varepsilon}$ the linearized current driven operator. We proved in [4] that $(\mathrm{CD})_{I, \varepsilon}$ has a unique solution. It is easy to check that a proof similar to the proof of uniqueness for $(\mathrm{CD})_{I, \varepsilon}$ allows us to show that for any functions $(a, b, c)$ satisfying (3.1)-(3.2) the kernel of $A_{(a, b, c)}^{\varepsilon}$ reduces to $\{0\}$. Since $A_{(a, b, c)}^{\varepsilon}$ is a Fredholm operator of index 0 , we deduce that $A_{(a, b, c)}^{\varepsilon}$ is boundedly invertible. We also set

$$
B_{(a, b, c)}^{\varepsilon}=i_{c} \circ\left(A_{(a, b, c)}^{\varepsilon}\right)^{-1},
$$

where $i_{c}$ denotes the canonical (continuous) injection from $X$ in $\left(W^{1,1}(\Omega)\right)^{4}$ and $\mathcal{L}(E, F)$ stands for the space of continuous linear applications from $E$ to $F$. We give in the next theorem a bound of the inverse of $A_{(a, b, c)}^{\varepsilon}$.

Theorem 3.1. Assume that $(a, b, c)$ satisfies (3.1)-(3.2) and let $R=\left(R_{i}\right)_{1 \leq i \leq 9} \in$ $Y$ be given. Then $U_{\varepsilon}=\left(A_{(a, b, c)}^{\varepsilon}\right)^{-1} R=\left(u_{i, \varepsilon}\right)_{1 \leq i \leq 4}$ satisfies the following estimates:

$$
\begin{gathered}
\left.\left\|u_{1, \varepsilon}\right\|_{1,1, \Omega} \leq c\left(\mathcal{P}_{M}\right) r_{-}^{-1} C\left(\left\|a^{\prime}\right\|_{0,1, \Omega}\right)\right)\|R\|_{Y}, \\
\left\|u_{i, \varepsilon}\right\|_{0, \infty, \Omega} \leq c\left(\mathcal{P}_{M}\right)\left(C\left(\left\|a^{\prime}\right\|_{0,1, \Omega}\right)\right)^{2}\|R\|_{Y} \quad \text { for } \quad i=2,3, \\
\left\|u_{i, \varepsilon}^{\prime}\right\|_{0,1, \Omega} \leq c\left(\mathcal{P}_{M}\right)\left(C\left(\left\|a^{\prime}\right\|_{0,1, \Omega}\right)\right)^{2} \\
\times\left(\left\|a^{\prime}\right\|_{0,1, \Omega}+1+\left(1+r_{-}^{-1}\right)\left\|d_{i}\right\|_{0, \infty, \Omega}\right)\|R\|_{Y} \quad \text { for } \quad i=2,3, \\
\left\|u_{4, \varepsilon}\right\|_{1,1, \Omega} \leq c\left(\mathcal{P}_{M}\right) C\left(\left\|a^{\prime}\right\|_{0,1, \Omega}\right)\|R\|_{Y},
\end{gathered}
$$




$$
\begin{aligned}
& \left\|u_{1, \varepsilon}^{\prime \prime}\right\|_{1,1, \Omega, \mathcal{P}_{M}} \leq c\left(\mathcal{P}_{M}\right) \varepsilon^{-1}\left(C\left(\left\|a^{\prime}\right\|_{0,1, \Omega}\right)\right)^{2} \\
& \times\left(\left\|a^{\prime}\right\|_{0,1, \Omega}+1+\left(1+r_{-}^{-1}\right)\left(\|b\|_{0, \infty, \Omega}+\|c\|_{0, \infty, \Omega}\right)\right)\|R\|_{Y},
\end{aligned}
$$

where $d_{2}=b, d_{3}=c, c\left(\mathcal{P}_{M}\right)$ is a generic constant which depends only on $c\left(\mathcal{P}_{M}\right)$ and

$$
C(t)=(1+t) \exp (t)
$$

Then we deduce from theorem 3.1 the following corollary.

Corollary 3.2. Assume that $(a, b, c)$ satisfies (3.1)-(3.2) and let $\mathcal{P}_{M}=$ $\left(a_{i}\right)_{0 \leq i \leq M+1}$ be a partition of $\bar{\Omega}$. Then there exists a constant $K$, which depends only on $M,\left\|a^{\prime}\right\|_{0,1, \Omega},\|b\|_{0, \infty, \Omega},\|c\|_{0, \infty, \Omega}$ and $r_{-}$, such that the family of operators $\left(B_{(a, b, c)}^{\varepsilon}\right)_{\varepsilon>0}$ satisfies

$$
\left\|B_{(a, b, c)}^{\varepsilon}\right\|_{\mathcal{L}\left(Y,\left(W^{1,1}(\Omega)\right)^{4}\right)} \leq K \quad \forall \varepsilon>0 .
$$

By extension, we still call $B_{(a, b, c)}^{\varepsilon}$ the inverse of the linearized current driven operator. Hence corollary 3.2 provides a uniform bound with respect to $\varepsilon$ for the inverse of the linearized current driven operator.

The next theorem gives the asymptotic error estimates.

Theorem 3.3. Assume that $N \in W_{d}^{3, \infty}\left(\Omega, \mathcal{P}_{M}\right)$ and let $I_{0} \in \mathcal{E}\left(N, \delta, \mathcal{P}_{M}\right)$ be given. For every compact subset $K$ of $\mathcal{O}\left(I_{0}, N, \delta, \mathcal{P}_{M}\right)$ we define $\Delta_{0}, \varepsilon_{1}$ and $r_{-}$as in lemma 2.6 and choose $\Delta \in\left(0, \Delta_{0}\right]$. Then there exists $C=C\left(K, I_{0}, N, \delta, \mathcal{P}_{M}\right)>0$ such that the following estimates hold:

$$
\begin{aligned}
& \left\|\left(\frac{\partial^{q} \psi_{\varepsilon}}{\partial I^{q}}-\frac{\partial^{q} \tilde{\psi}_{\varepsilon}}{\partial I^{q}}\right)(., I)\right\|_{1,1, \Omega}+\left\|\left(\frac{\partial^{q} n_{\varepsilon}}{\partial I^{q}}-\frac{\partial^{q} \tilde{n}_{\varepsilon}}{\partial I^{q}}\right)(., I)\right\|_{0, \infty, \Omega} \\
& +\left\|\left(\frac{\partial^{q} p_{\varepsilon}}{\partial I^{q}}-\frac{\partial^{q} \tilde{p}_{\varepsilon}}{\partial I^{q}}\right)(., I)\right\|_{0, \infty, \Omega}+\left|\left(\frac{\partial^{q} J_{n, \varepsilon}}{\partial I^{q}}-\frac{\partial^{q} J_{n}}{\partial I^{q}}\right)(I)\right| \\
& \leq C \sqrt{\varepsilon} \quad \forall \varepsilon \in\left(0, \varepsilon_{1}\right], \quad \forall I \in K, \quad q \in\{0,1\}
\end{aligned}
$$

where $\left(\psi_{\varepsilon}, n_{\varepsilon}, p_{\varepsilon}, J_{n, \varepsilon}\right)(., I)$ (resp. $\left(\psi, n, p, J_{n}(., I)\right)$ is the solution of $(C D)_{I, \varepsilon}$ (resp. $\left.(R C D)_{I}\right)$ and where $\tilde{\psi}_{\varepsilon}, \tilde{n}_{\varepsilon}$ and $\tilde{p}_{\varepsilon}$ are respectively defined by (2.66), (2.67) and (2.68).

This result can be easily extended (at the price of tedious computations) to the case $q \in \mathbb{N}$. In the same way higher order estimates (with respect to $\varepsilon$ ) can be derived by using higher order terms of the modified asymtotic expansion.

The next theorem and corollary give the multiplicity results for the voltage driven equations.

Theorem 3.4. Assume that $N \in W_{d}^{3, \infty}\left(\Omega, \mathcal{P}_{M}\right), \delta>0$ and $\mathcal{P}_{M}$ are such that there exists $V_{0}>0$ (resp. $<0$ ) satisfying $\#\left(\mathcal{S}\left(V_{0}\right) \cap \mathcal{S}_{n c}\right)=k \geq 2$. Then there exists $\varepsilon_{2}=\varepsilon_{2}\left(N, \delta, \mathcal{P}_{M}\right)>0$ such that for all $\varepsilon \in\left(0, \varepsilon_{2}\right)$, there exist $V_{-, \varepsilon} \in\left(0, V_{0}\right)$ (resp. $\left(-\infty, V_{0}\right)$ ) and $V_{+, \varepsilon} \in\left(V_{0},+\infty\right)$ (resp. $\left.\left(V_{0}, 0\right)\right)$ if $V_{0}>0$ (resp. $\left.<0\right)$, such that $(V D)_{\bar{V}, \varepsilon}$ has at least $k$ solutions in $\left(H^{1}(\Omega)\right)^{3} \times \mathbb{R}^{2}$ for all $\bar{V} \in\left(V_{-, \varepsilon}, V_{+, \varepsilon}\right)$.

It should be noted that the condition $\#\left(\mathcal{S}\left(V_{0}\right) \cap \mathcal{S}_{n c}\right)=k \geq 2$ can be reformulated in an equivalent way: (RVD) $V_{0}$ has $k$ isolated solutions, $k \geq 2$. Moreover, thanks to theorem 3.3, it is also possible to derive multiplicity results in the case where $(\mathrm{RVD})_{V_{0}}$ has several solutions which are not necessarily isolated. 
Corollary 3.5. Assume that $N \in W_{d}^{3, \infty}\left(\Omega, \mathcal{P}_{M}\right), \delta>0$ and $\mathcal{P}_{M}$ are such that

$$
\mathcal{C}_{\text {sat }, l}\left(N, \delta, \mathcal{P}_{M}\right) \cap \mathbb{R}^{\nu} \neq \emptyset, \mathcal{C}_{\text {sat }, r}\left(N, \delta, \mathcal{P}_{M}\right) \cap \mathbb{R}^{\nu} \neq \emptyset,
$$

where $\nu=+$ or $\nu=-$. Then there exists $\varepsilon_{2}=\varepsilon_{2}\left(N, \delta, \mathcal{P}_{M}\right)>0$ such that for all $\varepsilon \in\left(0, \varepsilon_{2}\right)$, there exist $\left(V_{-, \varepsilon}, V_{+, \varepsilon}\right) \in \mathbb{R}^{\nu} \times \mathbb{R}^{\nu}, V_{-, \varepsilon}<V_{+, \varepsilon}$ such that $(V D)_{\bar{V}, \varepsilon}$ has at least three solutions in $\left(H^{1}(\Omega)\right)^{3} \times \mathbb{R}^{2}$ for all $\bar{V} \in\left(V_{-, \varepsilon}, V_{+, \varepsilon}\right)$.

\section{Proof of the Main Results}

4.1. Proof of the estimate of the inverse of the linearized current driven operator. The proof of theorem 3.1 is divided into two steps. In the first step we prove that the estimates (3.5)-(3.9) hold in the case where $(a, b, c) \in(\mathcal{A}(\bar{\Omega}))^{3}$ and $R \in \mathcal{A}\left(\bar{\Omega}, \mathcal{P}_{M}\right) \times(\mathcal{A}(\bar{\Omega}))^{3} \times \mathbb{R}^{5}$. In the second step we prove these estimates under the hypotheses of theorem 3.1 by a density argument.

First step. Assume that $(a, b, c) \in(\mathcal{A}(\bar{\Omega}))^{3}$ and $R \in \mathcal{A}\left(\bar{\Omega}, \mathcal{P}_{M}\right) \times(\mathcal{A}(\bar{\Omega}))^{3} \times \mathbb{R}^{5}$. From classical results on ordinary differential equations we deduce that $U_{\varepsilon} \in X \cap$ $\left(\mathcal{A}\left(\bar{\Omega}, \mathcal{P}_{M}\right)^{3} \times \mathcal{A}(\bar{\Omega})\right)$. By the definition of $A_{(a, b, c)}^{\varepsilon}, U_{\varepsilon}$ satisfies the following system of equations:

$$
\begin{gathered}
\varepsilon u_{1, \varepsilon}^{\prime \prime}=u_{2, \varepsilon}-u_{3, \varepsilon}+R_{1}, \\
u_{2, \varepsilon}^{\prime}=a^{\prime} u_{2, \varepsilon}+b u_{1, \varepsilon}^{\prime}+u_{4, \varepsilon}+R_{2}, \\
u_{3, \varepsilon}^{\prime}=-a^{\prime} u_{3, \varepsilon}-c u_{1, \varepsilon}^{\prime}+u_{4, \varepsilon}+R_{3}, \\
u_{4, \varepsilon}^{\prime}=R_{4}, \\
u_{1, \varepsilon}(-1)=R_{5}, \\
u_{2, \varepsilon}(-1)=R_{6}, \quad u_{2, \varepsilon}(1)=R_{7}, \quad u_{3, \varepsilon}(-1)=R_{8}, \quad u_{3, \varepsilon}(1)=R_{9} .
\end{gathered}
$$

Since (4.4) holds, we have

$$
u_{4, \varepsilon}(x)=\int_{-1}^{x} R_{4}(t) d t+j_{\varepsilon}
$$

where $j_{\varepsilon}$ is an unknown constant. We can assume without loss of generality that

$$
j_{\varepsilon} \geq 0 \text {. }
$$

For $x \in \bar{\Omega}$ we set

$$
\begin{gathered}
\rho_{\varepsilon}(x)=u_{1, \varepsilon}^{\prime}(x), \\
v_{\varepsilon}(x)=u_{2, \varepsilon}(x) H\left(\rho_{\varepsilon}(x)\right)+u_{3, \varepsilon}(x)\left(1-H\left(\rho_{\varepsilon}(x)\right)\right), \\
r_{\varepsilon}(x)=b(x) H\left(\rho_{\varepsilon}(x)\right)+c(x)\left(1-H\left(\rho_{\varepsilon}(x)\right)\right), \\
D_{\varepsilon}(x)=R_{2}(x) H\left(\rho_{\varepsilon}(x)\right)+R_{3}(x)\left(1-H\left(\rho_{\varepsilon}(x)\right)\right),
\end{gathered}
$$

where $H$ stands for the Heaviside function.

Since $\rho_{\varepsilon} \in \mathcal{A}\left(\bar{\Omega}, \mathcal{P}_{M}\right) \cap \mathcal{C}^{0}(\bar{\Omega})$, we can define the set of points of $\Omega$ at which $\rho_{\varepsilon}$ changes sign. Of course this set is finite (eventually empty). We denote by 
$\left(x_{i, \varepsilon}\right)_{1 \leq i \leq m_{\varepsilon}}$ its elements ordered in a strictly increasing sequence. Moreover we set $x_{0, \varepsilon}=-1$ and $x_{m_{\varepsilon}+1, \varepsilon}=1$, and we define $\alpha_{\varepsilon} \in W^{1,1}(\Omega)$ by

$$
\alpha_{\varepsilon}(x)=\int_{-1}^{x} \operatorname{sign}\left(\rho_{\varepsilon}(t)\right) a^{\prime}(t) d t
$$

Since $v_{\varepsilon} \in L^{\infty}(\Omega)$ and $\alpha_{\varepsilon} \in W^{1,1}(\Omega)$, we have $v_{\varepsilon} \exp \left(-\alpha_{\varepsilon}\right) \in \mathcal{D}^{\prime}(\Omega)$. Using now (4.1)-(4.3), since $\alpha_{\varepsilon} \in \mathcal{C}^{0}(\bar{\Omega})$ we deduce that

$$
\begin{aligned}
& \left(v_{\varepsilon} \exp \left(-\alpha_{\varepsilon}\right)\right)^{\prime}=\exp \left(-\alpha_{\varepsilon}\right)\left(\left|\rho_{\varepsilon}\right| r_{\varepsilon}+u_{4, \varepsilon}+D_{\varepsilon}\right) \\
& +\sum_{i=1}^{i=m_{\varepsilon}} \exp \left(-\alpha_{\varepsilon}\left(x_{i, \varepsilon}\right)\right)\left[v_{\varepsilon}\right]_{x_{i, \varepsilon}} \delta_{x_{i, \varepsilon}} \quad \text { in } \quad \mathcal{D}^{\prime}(\Omega),
\end{aligned}
$$

where $[f]_{x}=f\left(x^{+}\right)-f\left(x^{-}\right)$and where $\delta_{x}$ denotes the Dirac distribution at $x$.

Since $\left(\rho_{\varepsilon}\right)_{/\left[a_{i}, a_{i+1}\right]} \in \mathcal{A}\left(\left[a_{i}, a_{i+1}\right]\right)$ for all $i \in\{0, \ldots, M\}$, we can define $k_{\varepsilon}$ as the first integer $k$ (which is necessarily odd, since $\rho_{\varepsilon}$ continuously changes sign at $x_{i, \varepsilon}$ ) such that $\rho_{\varepsilon}^{\left(k_{\varepsilon}\right)}\left(x_{i, \varepsilon}^{+}\right) \neq 0$ (or equivalently $\rho_{\varepsilon}^{\left(k_{\varepsilon}\right)}\left(x_{i, \varepsilon}^{-}\right) \neq 0$ ). We set

$$
\beta_{i, \varepsilon}=\operatorname{sign}\left(\rho_{\varepsilon}^{\left(k_{\varepsilon}\right)}\left(x_{i, \varepsilon}^{+}\right)\right) \in\{-1,1\} .
$$

Of course we also have

$$
\beta_{i, \varepsilon}=\operatorname{sign}\left(\rho_{\varepsilon}^{\left(k_{\varepsilon}\right)}\left(x_{i, \varepsilon}^{-}\right)\right) .
$$

We now use the definition (4.10) of $v_{\varepsilon}$. This, together with (4.1), (4.9) and (4.15), yields

$$
\left[v_{\varepsilon}\right]_{x_{i, \varepsilon}}=\beta_{i, \varepsilon}\left(\varepsilon \rho_{\varepsilon}^{\prime}\left(x_{i, \varepsilon}^{+}\right)-R_{1}\left(x_{i, \varepsilon}^{+}\right)\right) .
$$

In order to reformulate the equation (4.14) we introduce the following functions:

$$
G_{\varepsilon}(x)=\int_{-1}^{x}\left(\left|\rho_{\varepsilon}(t)\right| r_{\varepsilon}(t)+u_{4, \varepsilon}(t)+D_{\varepsilon}(t)\right) \exp \left(-\alpha_{\varepsilon}(t)\right) d t,
$$

$$
\theta_{\varepsilon}(x)=\left(v_{\varepsilon} \exp \left(-\alpha_{\varepsilon}\right)\right)(x)-G_{\varepsilon}(x)+\sum_{i=1}^{i=m_{\varepsilon}} \exp \left(-\alpha_{\varepsilon}\left(x_{i, \varepsilon}\right)\right) \beta_{i, \varepsilon} R_{1}\left(x_{i, \varepsilon}^{+}\right) H\left(x-x_{i, \varepsilon}\right) .
$$

Using now (4.16)-(4.18) in the equation (4.14), we obtain

$$
\theta_{\varepsilon}^{\prime}=\varepsilon \sum_{i=1}^{i=m_{\varepsilon}} \exp \left(-\alpha_{\varepsilon}\left(x_{i, \varepsilon}\right)\right) \beta_{i, \varepsilon} \rho_{\varepsilon}^{\prime}\left(x_{i, \varepsilon}^{+}\right) \delta_{x_{i, \varepsilon}} \quad \text { in } \quad \mathcal{D}^{\prime}(\Omega)
$$

We remark that by the definition of $\beta_{i, \varepsilon}$ we have $\beta_{i, \varepsilon} \rho_{\varepsilon}^{\prime}\left(x_{i, \varepsilon}^{+}\right)=\left|\rho_{\varepsilon}^{\prime}\left(x_{i, \varepsilon}^{+}\right)\right|$. This, together with (4.19), implies that

$$
\theta_{\varepsilon}^{\prime} \geq 0 \quad \text { in } \quad \mathcal{D}^{\prime}(\Omega)
$$

Hence $\theta_{\varepsilon}$ is an increasing function in $\bar{\Omega}$. Going back to the definition (4.18) of $\theta_{\varepsilon}$ we have

$$
\begin{aligned}
& \exp \left(\alpha_{\varepsilon}(x)\right)\left(v_{\varepsilon}(-1)+G_{\varepsilon}(x)-K_{1, \varepsilon}(x)\right) \leq v_{\varepsilon}\left(x^{ \pm}\right) \\
& \leq \exp \left(\alpha_{\varepsilon}(x)\right)\left(v_{\varepsilon}(1) \exp \left(-\alpha_{\varepsilon}(1)\right)+G_{\varepsilon}(x)-G_{\varepsilon}(1)+K_{2, \varepsilon}(x)\right) \quad \forall x \in \bar{\Omega},
\end{aligned}
$$
where $K_{i, \varepsilon}, i=1,2$, are defined by

$$
K_{1, \varepsilon}(x)=\sum_{i=1}^{i=m_{\varepsilon}} \exp \left(-\alpha_{\varepsilon}\left(x_{i, \varepsilon}\right)\right) \beta_{i, \varepsilon} R_{1}\left(x_{i, \varepsilon}^{+}\right) H\left(x-x_{i, \varepsilon}\right),
$$




$$
K_{2, \varepsilon}(x)=\sum_{i=1}^{i=m_{\varepsilon}} \exp \left(-\alpha_{\varepsilon}\left(x_{i, \varepsilon}\right)\right) \beta_{i, \varepsilon} R_{1}\left(x_{i, \varepsilon}^{+}\right)\left(1-H\left(x-x_{i, \varepsilon}\right)\right) .
$$

In order to prove the estimates (3.5)-(3.9) we must estimate the different terms involved in the left and right hand sides of (4.20). For the sake of clearness we give these various estimates in the next lemma. Keeping the above notations, we have

Lemma 4.1. Assume that $(a, b, c) \in(\mathcal{A}(\bar{\Omega}))^{3}$ and that $(b, c)$ satisfies (3.2). Let moreover $R \in \mathcal{A}\left(\bar{\Omega}, \mathcal{P}_{M}\right) \times(\mathcal{A}(\bar{\Omega}))^{3} \times \mathbb{R}^{5}$ be given. Then the following estimates hold:

$$
\max \left(\left\|\alpha_{\varepsilon}^{\prime}\right\|_{0,1, \Omega},\left|\alpha_{\varepsilon}(x)-\alpha_{\varepsilon}(t)\right|\right) \leq\left\|a^{\prime}\right\|_{0,1, \Omega} \quad \forall(x, t) \in(\bar{\Omega})^{2},
$$

$\left|K_{i, \varepsilon}(x)\right| \exp \left(\alpha_{\varepsilon}(t)\right) \leq c\left(\mathcal{P}_{M}\right) C\left(\left\|a^{\prime}\right\|_{0,1, \Omega}\right)\left\|R_{1}\right\|_{1,1, \Omega, \mathcal{P}_{M}} \quad \forall(x, t) \in(\bar{\Omega})^{2}, i \in\{1,2\}$,

$$
G_{\varepsilon}(x) \exp \left(\alpha_{\varepsilon}(x)\right) \geq-2 \exp \left(\left\|a^{\prime}\right\|_{0,1, \Omega}\right)\left(\sum_{i=2}^{i=4}\left\|R_{i}\right\|_{0,1, \Omega}\right) \quad \forall x \in \bar{\Omega}
$$

$$
\left(G_{\varepsilon}(x)-G_{\varepsilon}(1)\right) \exp \left(\alpha_{\varepsilon}(x)\right) \leq 2 \exp \left(\left\|a^{\prime}\right\|_{0,1, \Omega}\right)\left(\sum_{i=2}^{i=4}\left\|R_{i}\right\|_{0,1, \Omega}\right) \quad \forall x \in \bar{\Omega},
$$

$r_{-}\left\|\rho_{\varepsilon}\right\|_{0,1, \Omega}+2\left|j_{\varepsilon}\right| \leq G_{\varepsilon}(1) \exp \left(\alpha_{\varepsilon}\left(t_{1, \varepsilon}\right)\right)+2 \exp \left(\alpha_{\varepsilon}\left(t_{1, \varepsilon}\right)-\alpha_{\varepsilon}\left(t_{0, \varepsilon}\right)\right) \sum_{i=2}^{i=4}\left\|R_{i}\right\|_{0,1, \Omega}$, where $C($.$) is defined by (3.10) and where t_{i, \varepsilon}, i=0,1$, are such that

$$
\alpha_{\varepsilon}\left(t_{0, \varepsilon}\right)=\min _{t \in \bar{\Omega}}\left(\alpha_{\varepsilon}(t)\right), \quad \alpha_{\varepsilon}\left(t_{1, \varepsilon}\right)=\max _{t \in \bar{\Omega}}\left(\alpha_{\varepsilon}(t)\right) .
$$

Proof. The first estimate (4.23) follows easily from (4.13). We prove (4.24) as follows. For $x \in \bar{\Omega}$ we denote by $l_{\varepsilon} \in\left\{1, \ldots, m_{\varepsilon}-1\right\}$ the integer (which depends on $x$ ) such that $x \in\left[x_{l_{\varepsilon}, \varepsilon}, x_{l_{\varepsilon}+1, \varepsilon}\right)$. Moreover we denote by $i_{0, \varepsilon} \in\{0, \ldots, M\}$ the integer (which depends on $x$ ) such that $x_{l_{\varepsilon}+1, \varepsilon} \in\left[a_{i_{0, \varepsilon}}, a_{i_{0, \varepsilon}+1}\right.$ ). Hence we have

$$
\begin{aligned}
& \left|K_{2, \varepsilon}(x)\right| \exp \left(\alpha_{\varepsilon}(t)\right)=\left|\sum_{i=l_{\varepsilon}+1}^{m_{\varepsilon}} \exp \left(\alpha_{\varepsilon}(t)-\alpha_{\varepsilon}\left(x_{i, \varepsilon}\right)\right) \beta_{i, \varepsilon} R_{1}\left(x_{i, \varepsilon}^{+}\right)\right| \\
& \leq \sum_{l=i_{0, \varepsilon}}^{M}\left|\sum_{i \geq l_{\varepsilon}+1 / x_{i, \varepsilon} \in\left[a_{l}, a_{l+1}\right)} \exp \left(\alpha_{\varepsilon}(t)-\alpha_{\varepsilon}\left(x_{i, \varepsilon}\right)\right) \beta_{i, \varepsilon} R_{1}\left(x_{i, \varepsilon}^{+}\right)\right| \\
& \leq \sum_{l=0}^{M}\left|\sum_{i \geq l_{\varepsilon}+1 / x_{i, \varepsilon} \in\left[a_{l}, a_{l+1}\right)} \exp \left(\alpha_{\varepsilon}(t)-\alpha_{\varepsilon}\left(x_{i, \varepsilon}\right)\right) \beta_{i, \varepsilon} R_{1}\left(x_{i, \varepsilon}^{+}\right)\right| \quad \forall(x, t) \in(\bar{\Omega})^{2} .
\end{aligned}
$$

Since $\beta_{i, \varepsilon} \beta_{i+1, \varepsilon}<0$ and $\beta_{i, \varepsilon} \in\{-1,1\}$, we deduce that

$$
\begin{aligned}
& \left|\sum_{i \geq l_{\varepsilon}+1 / x_{i, \varepsilon} \in\left[a_{l}, a_{l+1}\right)} \exp \left(\alpha_{\varepsilon}(t)-\alpha_{\varepsilon}\left(x_{i, \varepsilon}\right)\right) \beta_{i, \varepsilon} R_{1}\left(x_{i, \varepsilon}^{+}\right)\right| \\
& \leq \exp \left(\alpha_{\varepsilon}(t)\right) B V_{\left[a_{l}, a_{l+1}\right]}\left(\exp \left(-\alpha_{\varepsilon}\right) R_{1}\right)+\exp \left(\alpha_{\varepsilon}(t)\right)|| \exp \left(-\alpha_{\varepsilon}\right) R_{1} \|_{0, \infty,\left[a_{l}, a_{l+1}\right]},
\end{aligned}
$$


where $B V_{[a, b]}(h)$ denotes the total variation of the function $h$ on the interval $[a, b]$. Using (4.23) and the above estimate in (4.28), we obtain (4.24) for $i=2$. The proof of (4.24) for $i=1$ is similar.

We prove (4.25) as follows. Using the assumption (3.2) together with (4.11), we have

$$
r_{\varepsilon}(x) \geq r_{-}>0 \quad \forall x \in \bar{\Omega} .
$$

This, combined with (4.7)-(4.8), yields

$$
\left|\rho_{\varepsilon}(t)\right| r_{\varepsilon}(t)+u_{4, \varepsilon}(t)+D_{\varepsilon}(t) \geq-\|\left. R_{4}\right|_{0,1, \Omega}-\left|D_{\varepsilon}(t)\right| \quad \forall t \in \bar{\Omega} .
$$

Using this last inequality, together with (4.12)-(4.13), in (4.17), we deduce (4.25). We prove in the same way that (4.26) holds. Finally, using once again the assumption (3.2) together with (4.8) in the expression which gives $G_{\varepsilon}(1)$, we obtain (4.27). This concludes the proof of the lemma.

Thanks to this lemma we are now able to prove the estimates (3.5)-(3.9). We first prove (3.5) as follows. We set $x=-1$ in (4.20). This gives

$$
G_{\varepsilon}(1) \leq v_{\varepsilon}(1) \exp \left(-\alpha_{\varepsilon}(1)\right)-v_{\varepsilon}(-1)+K_{1, \varepsilon}(1)
$$

This, together with (4.27), yields

$$
\begin{aligned}
& r_{-}\left\|\rho_{\varepsilon}\right\|_{0,1, \Omega}+2\left|j_{\varepsilon}\right| \leq v_{\varepsilon}(1) \exp \left(\alpha_{\varepsilon}\left(t_{1, \varepsilon}\right)-\alpha_{\varepsilon}(1)\right)-v_{\varepsilon}(-1) \exp \left(\alpha_{\varepsilon}\left(t_{1, \varepsilon}\right)\right) \\
& +K_{1, \varepsilon}(1) \exp \left(\alpha_{\varepsilon}\left(t_{1, \varepsilon}\right)\right)+2 \exp \left(\alpha_{\varepsilon}\left(t_{1, \varepsilon}\right)-\alpha_{\varepsilon}\left(t_{0, \varepsilon}\right)\right) \sum_{i=2}^{i=4}\left\|R_{i}\right\|_{0,1, \Omega} .
\end{aligned}
$$

We now use the resulting estimate, together with (4.23), (4.24) and the boundary conditions (4.6). This gives

$$
r_{-}\left\|\rho_{\varepsilon}\right\|_{0,1, \Omega}+2\left|j_{\varepsilon}\right| \leq c\left(\mathcal{P}_{M}\right) C\left(\left\|a^{\prime}\right\|_{0,1, \Omega}\right)\|R\|_{Y}
$$

which together with the boundary condition (4.5) gives (3.5). We now prove (3.6) as follows. We first use the estimates (4.23)-(4.26) in the inequalities (4.20). This yields

$$
\left\|v_{\varepsilon}\right\|_{0, \infty, \Omega} \leq c\left(\mathcal{P}_{M}\right) C\left(\left\|a^{\prime}\right\|_{0,1, \Omega}\right)\|R\|_{Y} .
$$

Going back to the definition (4.10) of $v_{\varepsilon}$, we deduce that

$\left\|u_{2, \varepsilon}\right\|_{0, \infty,\left[x_{i, \varepsilon}, x_{i+1, \varepsilon}\right]} \leq c\left(\mathcal{P}_{M}\right) C\left(\left\|a^{\prime}\right\|_{0,1, \Omega}\right)\|R\|_{Y} \quad$ if $\quad \rho_{\varepsilon} \geq 0 \quad$ on $\quad\left[x_{i, \varepsilon}, x_{i+1, \varepsilon}\right]$,

$\left\|u_{3, \varepsilon}\right\|_{0, \infty,\left[x_{i, \varepsilon}, x_{i+1, \varepsilon}\right]} \leq c\left(\mathcal{P}_{M}\right) C\left(\left\|a^{\prime}\right\|_{0,1, \Omega}\right)\|R\|_{Y} \quad$ if $\quad \rho_{\varepsilon} \leq 0 \quad$ on $\quad\left[x_{i, \varepsilon}, x_{i+1, \varepsilon}\right]$.

These last two inequalities imply in particular

$$
\left|u_{j, \varepsilon}\left(x_{l, \varepsilon}\right)\right| \leq c\left(\mathcal{P}_{M}\right) C\left(\left\|a^{\prime}\right\|_{0,1, \Omega}\right)\|R\|_{Y}, \quad j=2,3 \quad \text { and } \quad \forall l \in\left\{0, \ldots, m_{\varepsilon}+1\right\} .
$$

Hence in order to prove (3.6) we must estimate $\left\|u_{2, \varepsilon}\right\|_{0, \infty,\left[x_{i, \varepsilon}, x_{i+1, \varepsilon}\right]}$ (resp. $\left.\left\|u_{3, \varepsilon}\right\|_{0, \infty,\left[x_{i, \varepsilon}, x_{i+1, \varepsilon}\right]}\right)$ on the intervals $\left[x_{i, \varepsilon}, x_{i+1, \varepsilon}\right]$ where $\rho_{\varepsilon} \leq 0$ (resp. $\left.\geq 0\right)$. Assume first that $\rho_{\varepsilon} \geq 0$ on $\left[x_{i, \varepsilon}, x_{i+1, \varepsilon}\right]$. Integrating (4.3) between $x_{i, \varepsilon}$ and $x$, we 
obtain

$$
\begin{aligned}
& u_{3, \varepsilon}(x)=u_{3, \varepsilon}\left(x_{i, \varepsilon}\right) \exp \left(a\left(x_{i, \varepsilon}\right)-a(x)\right)-\int_{x_{i, \varepsilon}}^{x} c(t) \rho_{\varepsilon}(t) \exp (a(t)-a(x)) d t \\
& +\int_{x_{i, \varepsilon}}^{x}\left(u_{4, \varepsilon}(t)+R_{3}(t)\right) \exp (a(t)-a(x)) d t .
\end{aligned}
$$

Since we assumed that $\rho_{\varepsilon} \geq 0$ on $\left[x_{i, \varepsilon}, x_{i+1, \varepsilon}\right]$ and since (3.2) holds, we deduce from the above equation that

$$
u_{3, \varepsilon}(x) \leq \exp \left(\left\|a^{\prime}\right\|_{0,1, \Omega}\right)\left(\left|u_{3, \varepsilon}\left(x_{i, \varepsilon}\right)\right|+\left\|u_{4, \varepsilon}\right\|_{0,1, \Omega}+\left\|R_{3}\right\|_{0,1, \Omega}\right) \quad \forall x \in\left[x_{i, \varepsilon}, x_{i+1, \varepsilon}\right] .
$$

Using now (4.7), (4.28), and (4.31) (with $j=3$ and $l=i$ ) in this last estimate, we obtain

$$
u_{3, \varepsilon}(x) \leq c\left(\mathcal{P}_{M}\right)\left(C\left(\left\|a^{\prime}\right\|_{0,1, \Omega}\right)\right)^{2}\|R\|_{Y} \quad \forall x \in\left[x_{i, \varepsilon}, x_{i+1, \varepsilon}\right] .
$$

In a similar way, integration of (4.3) between $x$ and $x_{i+1, \varepsilon}$, together with (4.7), (4.28) and (4.31) (with $j=3, l=i+1$ ), yields

$$
u_{3, \varepsilon}(x) \geq-c\left(\mathcal{P}_{M}\right)\left(C\left(\left\|a^{\prime}\right\|_{0,1, \Omega}\right)\right)^{2}\|R\|_{Y} \quad \forall x \in\left[x_{i, \varepsilon}, x_{i+1, \varepsilon}\right] .
$$

Hence we have proved that

$$
\left\|u_{3, \varepsilon}\right\|_{0, \infty,\left[x_{i, \varepsilon}, x_{i+1, \varepsilon}\right]} \leq c\left(\mathcal{P}_{M}\right)\left(C\left(\left\|a^{\prime}\right\|_{0,1, \Omega}\right)\right)^{2}\|R\|_{Y} \text { if } \rho_{\varepsilon} \geq 0 \text { on }\left[x_{i, \varepsilon}, x_{i+1, \varepsilon}\right] .
$$

Using (4.2) we prove in a similar way that

$$
\left\|u_{2, \varepsilon}\right\|_{0, \infty,\left[x_{i, \varepsilon}, x_{i+1, \varepsilon}\right]} \leq c\left(\mathcal{P}_{M}\right)\left(C\left(\left\|a^{\prime}\right\|_{0,1, \Omega}\right)\right)^{2}\|R\|_{Y} \text { if } \rho_{\varepsilon} \leq 0 \text { on }\left[x_{i, \varepsilon}, x_{i+1, \varepsilon}\right] .
$$

These last two estimates, together with (4.29) and (4.30), give (3.6). Moreover, using (3.6) in (4.2) and (4.3), we derive (3.7). The inequality (3.8) follows easily from (4.7) and (4.28). Finally, using (3.6), (3.7) in (4.1), we obtain (3.9). This concludes the first step of the proof.

Second step Assume now that $(a, b, c) \in\left(W^{1,1}(\Omega)\right)^{3}$ and that $R \in Y$. From the Stone-Weierstrass theorem and from the density of $\mathcal{C}^{0}([a, b])$ in $L^{1}([a, b])$ we deduce that there exist sequences $\left(a_{n}, b_{n}, c_{n}\right) \in(\mathcal{A}(\bar{\Omega}))^{3}$ and $R_{n}=\left(R_{i, n}\right)_{1 \leq i \leq 9} \in$ $\mathcal{A}\left(\bar{\Omega}, \mathcal{P}_{M}\right) \times(\mathcal{A}(\bar{\Omega}))^{3} \times \mathbb{R}^{5}$ such that

$$
\begin{gathered}
\left\|a_{n}-a\right\|_{1,1, \Omega}+\left\|b_{n}-b\right\|_{1,1, \Omega}+\left\|c_{n}-c\right\|_{1,1, \Omega} \rightarrow 0 \quad \text { as } n \rightarrow+\infty, \\
\left\|R_{n}-R\right\|_{Y} \rightarrow 0 \quad \text { as } n \rightarrow+\infty .
\end{gathered}
$$

From this, since $(b, c)$ satisfies $(3.2)$, we deduce that

$$
\begin{array}{r}
\min \left(b_{n}(x), c_{n}(x)\right) \geq r_{-}-\max \left(\left\|b_{n}-b\right\|_{0, \infty, \Omega},\left\|c_{n}-c\right\|_{0, \infty, \Omega}\right)=r_{-, n}>0, \\
\forall x \in \bar{\Omega}, \quad \text { and for } n \text { sufficiently large. }
\end{array}
$$

Hence $\left(b_{n}, c_{n}\right)$ satisfies (3.2) for $n$ sufficiently large, with $r_{-}$replaced by $r_{-, n}$. We set $A_{n}^{\varepsilon}=A_{\left(a_{n}, b_{n}, c_{n}\right)}^{\varepsilon}$. Moreover we set $U_{n}^{\varepsilon}=\left(u_{i, \varepsilon, n}\right)_{1 \leq i \leq 4}=\left(A_{n}^{\varepsilon}\right)^{-1} R_{n}$. From the first step of the proof, we know that $U_{n}^{\varepsilon}$ satisfies the estimates (3.5)-(3.9) with $(a, b, c)$ and $r_{-}$respectively replaced by $\left(a_{n}, b_{n}, c_{n}\right)$ and $r_{-, n}$. Since the sequences $\left(a_{n}\right)_{n},\left(b_{n}\right)_{n},\left(c_{n}\right)_{n}$ are bounded in $W^{1,1}(\Omega)$, and since $r_{-, n} \rightarrow r_{-}$as $n$ goes to $+\infty$, we deduce that $\left(U_{n}^{\varepsilon}\right)_{n}$ is bounded in $X$. We then write the system satisfied by $U_{n}^{\varepsilon}-U_{m}^{\varepsilon}$. Using once again the estimates (3.5)-(3.9) of the first step, together with the boundedness of $\left(U_{n}^{\varepsilon}\right)_{n}$ in $X$, we deduce that $\left(U_{n}^{\varepsilon}\right)_{n}$ is a Cauchy sequence 
in $X$ which converges to $U_{\varepsilon}$ since the kernel of $A_{(a, b, c)}^{\varepsilon}$ reduces to $\{0\}$. We conclude this second step by letting $n$ goes to $+\infty$ in the estimates on $U_{n}^{\varepsilon}$.

4.2. Proof of the asymptotic estimates. In order to prove theorem 3.3 we need the following lemma.

Lemma 4.2. Assume the hypotheses of theorem 3.3. Then

$$
U_{\varepsilon}=\left(\psi_{\varepsilon}-\tilde{\psi}_{\varepsilon}, n_{\varepsilon}-\tilde{n}_{\varepsilon}, p_{\varepsilon}-\tilde{p}_{\varepsilon}, J_{n, \varepsilon}-J_{n}\right)=\left(u_{i, \varepsilon}\right)_{1 \leq i \leq 4} \in X
$$

and satisfies the equation

$$
A_{\left(\tilde{\psi}_{\varepsilon}, n_{\varepsilon}, p_{\varepsilon}\right)}^{\varepsilon}\left(U_{\varepsilon}\right)=R_{\varepsilon}(., I),
$$

where $A_{\left(\tilde{\psi}_{\varepsilon}, n_{\varepsilon}, p_{\varepsilon}\right)}$ is defined by (3.3) and where $R_{\varepsilon}=\left(R_{i, \varepsilon}\right)_{1 \leq i \leq 9} \in Y$ is given by

$$
\begin{aligned}
R_{1, \varepsilon}(x, I)= & -\varepsilon \psi^{\prime \prime}(x)-2 \sqrt{\varepsilon} \sum_{i \in P \cup Q \cup S_{l}} \frac{\partial \hat{\psi}_{i, \varepsilon}}{\partial \tau}\left(\tau_{i, \varepsilon}(x), I\right) M_{i, \Delta}^{\prime}(x) \\
& -\varepsilon \sum_{i \in P \cup Q \cup S_{l}} \hat{\psi}_{i, \varepsilon}\left(\tau_{i, \varepsilon}(x), I\right) M_{i, \Delta}^{\prime \prime}(x) \\
& +\sqrt{\varepsilon} \sum_{i \in P} \frac{\beta_{i}(I)}{2} \sqrt{\left|\left[N^{\prime}\right]_{a_{i}}\right|} \exp \left(-\sqrt{\left|\left[N^{\prime}\right]_{a_{i}}\right|}\left|\tau_{i, \varepsilon}(x)\right|\right) M_{i, \Delta}(x),
\end{aligned}
$$

$$
R_{i, \varepsilon}(x, I)=T_{i, \varepsilon}(x, I)-S_{i, \varepsilon}(x, I)-A_{i, \varepsilon}(x, I) \quad i=2,3,
$$

$$
R_{4, \varepsilon}(x, I)=0,
$$

The functions $T_{i, \varepsilon}, S_{i, \varepsilon}, A_{i, \varepsilon}$ for $i=2,3$ are given by

$$
\begin{aligned}
& T_{2, \varepsilon}(x, I)=\left(\sum_{j \in Q \cup S_{l}} n\left(a_{j}^{\nu}, I\right)\left(\exp \left(\hat{\psi}_{j, \varepsilon}\left(\tau_{j, \varepsilon}(x), I\right)\right)-1\right) M_{j, \Delta}(x)\right) \\
& \times\left(\psi^{\prime}(x, I)+\sum_{i \in P \cup Q \cup S_{l}} \hat{\psi}_{i, \varepsilon}\left(\tau_{i, \varepsilon}(x), I\right) M_{i, \Delta}^{\prime}(x)\right) \\
& +n(x, I)\left(\sum_{i \in P \cup Q \cup S_{l}} \hat{\psi}_{i, \varepsilon}\left(\tau_{i, \varepsilon}(x), I\right) M_{i, \Delta}^{\prime}(x)\right)
\end{aligned}
$$

$$
S_{2, \varepsilon}(x, I)=\sum_{i \in Q \cup S_{l}} n\left(a_{i}^{\nu}, I\right)\left(\exp \left(\hat{\psi}_{i, \varepsilon}\left(\tau_{i, \varepsilon}(x), I\right)\right)-1\right) M_{i, \Delta}^{\prime}(x),
$$




$$
\begin{aligned}
& A_{2, \varepsilon}(x, I)= \varepsilon^{-1 / 2} \sum_{i \in P \cup Q \cup S_{l}} M_{i, \Delta}(x) \frac{\partial \hat{\psi}_{i, \varepsilon}}{\partial \tau}\left(\tau_{i, \varepsilon}(x), I\right) \times\left[n\left(a_{i}^{\nu}, I\right) \exp \left(\hat{\psi}_{i, \varepsilon}\left(\tau_{i, \varepsilon}(x), I\right)\right)\right. \\
&\left.-n(x, I)-\sum_{j \in Q \cup S_{l}} n\left(a_{j}^{\nu}, I\right)\left(\exp \left(\hat{\psi}_{j, \varepsilon}\left(\tau_{j, \varepsilon}(x), I\right)\right)-1\right) M_{j, \Delta}(x)\right] \\
&(4.41) \quad-\varepsilon^{-1 / 2} \sum_{i \in P} M_{i, \Delta}(x) \frac{\partial \hat{\psi}_{i, \varepsilon}}{\partial \tau}\left(\tau_{i, \varepsilon}(x), I\right) n\left(a_{i}^{\nu}, I\right) \exp \left(\hat{\psi}_{i, \varepsilon}\left(\tau_{i, \varepsilon}(x), I\right)\right), \\
& T_{3, \varepsilon}(x, I)=-\left(\sum_{j \in Q \cup S_{l}} p\left(a_{j}^{\nu}, I\right)\left(\exp \left(-\hat{\psi}_{j, \varepsilon}\left(\tau_{j, \varepsilon}(x), I\right)\right)-1\right) M_{j, \Delta}(x)\right) \\
& \quad \times\left(\psi^{\prime}(x, I)+\sum_{i \in P \cup Q \cup S_{l}} \hat{\psi}_{i, \varepsilon}\left(\tau_{i, \varepsilon}(x), I\right) M_{i, \Delta}^{\prime}(x)\right) \\
& \quad p(x, I)\left(\sum_{i \in P \cup Q \cup S_{l}} \hat{\psi}_{i, \varepsilon}\left(\tau_{i, \varepsilon}(x), I\right) M_{i, \Delta}^{\prime}(x)\right) \\
& \quad S_{3, \varepsilon}(x, I)=\sum_{i \in Q \cup S_{l}} p\left(a_{i}^{\nu}, I\right)\left(\exp \left(-\hat{\psi}_{i, \varepsilon}\left(\tau_{i, \varepsilon}(x), I\right)\right)-1\right) M_{i, \Delta}^{\prime}(x),
\end{aligned}
$$

$$
\begin{aligned}
A_{3, \varepsilon}(x, I)= & \varepsilon^{-1 / 2} \sum_{i \in P \cup Q \cup S_{l}} M_{i, \Delta}(x) \frac{\partial \hat{\psi}_{i, \varepsilon}}{\partial \tau}\left(\tau_{i, \varepsilon}(x), I\right) \\
& \times\left[-p\left(a_{i}^{\nu}, I\right) \exp \left(-\hat{\psi}_{i, \varepsilon}\left(\tau_{i, \varepsilon}(x), I\right)\right)\right. \\
& \left.+p(x, I)+\sum_{j \in Q \cup S_{l}} p\left(a_{j}^{\nu}, I\right)\left(\exp \left(-\hat{\psi}_{j, \varepsilon}\left(\tau_{j, \varepsilon}(x), I\right)\right)-1\right) M_{j, \Delta}(x)\right] \\
& +\varepsilon^{-1 / 2} \sum_{i \in P} M_{i, \Delta}(x) \frac{\partial \hat{\psi}_{i, \varepsilon}}{\partial \tau}\left(\tau_{i, \varepsilon}(x), I\right) p\left(a_{i}^{\nu}, I\right) \exp \left(-\hat{\psi}_{i, \varepsilon}\left(\tau_{i, \varepsilon}(x), I\right)\right),
\end{aligned}
$$

Proof. By construction $\tilde{\psi}_{\varepsilon}(., I) \in W^{2,1}(\Omega)$. Moreover using proposition 2.5 and differentiating (2.66) twice with respect to $x$, we obtain

$$
\varepsilon \tilde{\psi}_{\varepsilon}^{\prime \prime}(x, I)=-R_{1, \varepsilon}(x, I)+\sum_{i \in Q \cup S_{l}} \frac{\partial^{2} \hat{\psi}_{\varepsilon}}{\partial \tau^{2}}\left(\tau_{i, \varepsilon}(x), I\right) M_{i, \Delta}(x),
$$

where $R_{1, \varepsilon}$ is defined as in (4.35). Since for $i \in Q \cup S_{l}, \hat{\psi}_{\varepsilon}$ and $\psi_{\varepsilon}$ satisfy respectively (2.13) and (1.1) and since $n$ and $p$ satisfy (2.3), we deduce easily that

$$
\varepsilon\left(\psi_{\varepsilon}-\tilde{\psi}_{\varepsilon}\right)^{\prime \prime}=n_{\varepsilon}-\tilde{n}_{\varepsilon}-\left(p_{\varepsilon}-\tilde{p}_{\varepsilon}\right)+R_{1, \varepsilon} .
$$


By construction $\tilde{n}_{\varepsilon}(., I) \in W^{1,1}(\Omega)$. Hence we can differentiate (2.67) with respect to $x$. Using (2.66), we then obtain

$$
\begin{aligned}
& \tilde{n}_{\varepsilon}^{\prime}(x, I)-\tilde{n}_{\varepsilon}(x, I) \tilde{\psi}_{\varepsilon}^{\prime}(x, I)=n^{\prime}(x, I)+S_{2, \varepsilon}(x, I) \\
& +\varepsilon^{-1 / 2} \sum_{i \in Q \cup S_{l}} M_{i, \Delta}(x) \frac{\partial \hat{\psi}_{i, \varepsilon}}{\partial \tau}\left(\tau_{i, \varepsilon}(x), I\right) n\left(a_{i}^{\nu}, I\right) \exp \left(\hat{\psi}_{i, \varepsilon}\left(\tau_{i, \varepsilon}(x), I\right)\right) \\
& \times-\left(n(x, I)+\sum_{j \in Q \cup S_{l}} M_{j, \Delta}(x) n\left(a_{j}^{\nu}, I\right)\left(\exp \left(\hat{\psi}_{j, \varepsilon}\left(\tau_{j, \varepsilon}(x), I\right)\right)-1\right)\right) \\
& \times\left(\psi^{\prime}(x, I)+\sum_{i \in P \cup Q \cup S_{l}} \hat{\psi}_{i, \varepsilon}\left(\tau_{i, \varepsilon}(x), I\right) M_{i, \Delta}^{\prime}(x)\right) \\
& \left.+\varepsilon^{-1 / 2} \sum_{i \in P \cup Q \cup S_{l}} \frac{\partial \hat{\psi}_{i, \varepsilon}}{\partial \tau}\left(\tau_{i, \varepsilon}(x), I\right) M_{i, \Delta}(x)\right),
\end{aligned}
$$

Since $\left(\psi, n, p, J_{n}\right)(., I)$ satisfies $(\mathrm{RCD})_{I}$, we have

$$
\tilde{n}_{\varepsilon}^{\prime}(x, I)-\tilde{n}_{\varepsilon}(x, I) \tilde{\psi}_{\varepsilon}^{\prime}(x, I)=J_{n}(I)+S_{2, \varepsilon}(x, I)-T_{2, \varepsilon}(x, I)+A_{2, \varepsilon}(x, I),
$$

where $S_{2, \varepsilon}, T_{2, \varepsilon}$ and $A_{2, \varepsilon}$ are respectively given by (4.39), (4.40) and (4.41). Subtracting this last equation from (1.2), we obtain

$$
\left(n_{\varepsilon}-\tilde{n}_{\varepsilon}\right)^{\prime}=\left(n_{\varepsilon}-\tilde{n}_{\varepsilon}\right) \tilde{\psi}_{\varepsilon}^{\prime}+n_{\varepsilon}\left(\psi_{\varepsilon}-\tilde{\psi}_{\varepsilon}\right)^{\prime}+J_{n, \varepsilon}-J_{n}+T_{2, \varepsilon}-S_{2, \varepsilon}-A_{2, \varepsilon} .
$$

In a similar way we obtain

$$
\left(p_{\varepsilon}-\tilde{p}_{\varepsilon}\right)^{\prime}=-\left(p_{\varepsilon}-\tilde{p}_{\varepsilon}\right) \tilde{\psi}_{\varepsilon}^{\prime}-p_{\varepsilon}\left(\psi_{\varepsilon}-\tilde{\psi}_{\varepsilon}\right)^{\prime}+J_{n, \varepsilon}-J_{n}+T_{3, \varepsilon}-S_{3, \varepsilon}-A_{3, \varepsilon} .
$$

Moreover we have $\left(J_{n, \varepsilon}-J_{n}\right)^{\prime}=0$ and $\left(\psi_{\varepsilon}-\tilde{\psi}_{\varepsilon}\right)(-1, I)=0,\left(n_{\varepsilon}-\tilde{n}_{\varepsilon}\right)( \pm 1, I)=$ $\left(p_{\varepsilon}-\tilde{p}_{\varepsilon}\right)( \pm 1, I)=0$. This concludes the proof of the lemma.

Lemma 4.3. Assume the hypotheses of theorem 3.3 and let $R_{\varepsilon}$ be as in theorem 3.3. Then for $I_{0} \in \mathcal{E}\left(N, \delta, \mathcal{P}_{M}\right)$ and for every compact subset $K$ of $\mathcal{O}\left(I_{0}, N, \delta, \mathcal{P}_{M}\right)$ the following estimates hold:

$$
\left\|\frac{\partial^{q} R_{\varepsilon}}{\partial I^{q}}(., I)\right\|_{Y} \leq C \sqrt{\varepsilon} \quad \forall \varepsilon \in\left(0, \varepsilon_{1}\right], \forall I \in K, q \in\{0,1\},
$$

where $\varepsilon_{1}$ is as in lemma 2.6 and $C=C\left(K, I_{0}, N, \delta, \mathcal{P}_{M}\right)$.

Proof. We first estimate $\left\|R_{1, \varepsilon}(., I)\right\|_{1,1, \Omega, \mathcal{P}_{M}}$ as follows.

Since $N \in W_{d}^{3, \infty}\left(\Omega, \mathcal{P}_{M}\right)$, we have $\psi \in W_{d}^{3, \infty}\left(\Omega, \mathcal{P}_{M}\right)$. Using the exponential decay of the functions $\hat{\psi}_{i, \varepsilon}(., I)$ at $\pm \infty$, we easily obtain

$$
\left\|R_{1, \varepsilon}(., I)\right\|_{1,1, \Omega, \mathcal{P}_{M}} \leq C \sqrt{\varepsilon} \quad \forall \varepsilon \in\left(0, \varepsilon_{1}\right], \quad \forall I \in K .
$$

We now estimate $R_{2, \varepsilon}$ as follows.

Since $M_{i, \Delta}^{\prime}=0$ in a neighbourhood of $a_{i}$ we have

$$
\begin{aligned}
& \left\|T_{2, \varepsilon}(., I)\right\| \|_{0,1, \Omega} \\
& \quad \leq\left(\sum_{j \in Q \cup S_{l}}\left|n\left(a_{j}^{\nu}, I\right)\right|\left\|M_{j, \Delta}\right\|_{0, \infty, \Omega}\left\|\exp \left(\hat{\psi}_{j, \varepsilon}\left(\tau_{j, \varepsilon}(.), I\right)\right)-1\right\|_{0,1, \Omega}\right) \\
& \quad \times\left(|\psi(., I)|_{1, \infty, \Omega, \mathcal{P}_{M}}+C_{s} \varepsilon^{s}\right)+C_{s} \varepsilon^{s} \quad \forall s>0,
\end{aligned}
$$


where $C_{s}$ is a constant which depends only on $s, K, I_{0}, N, \delta, \mathcal{P}_{M}$. Making the change of variable $\tau_{j, \varepsilon}=\tau_{j, \varepsilon}(x)$ in the expression $\left\|\exp \left(\hat{\psi}_{j, \varepsilon}\left(\tau_{j, \varepsilon}(.), I\right)\right)-1\right\|_{0,1, \Omega}$, we deduce that

$$
\left\|T_{2, \varepsilon}(., I)\right\|_{0,1, \Omega} \leq C \sqrt{\varepsilon} \quad \forall \varepsilon \in\left(0, \varepsilon_{1}\right], \forall I \in K .
$$

In a similar way we have

$$
\left\|S_{2, \varepsilon}(., I)\right\|_{0,1, \Omega} \leq C_{s} \varepsilon^{s} \quad \forall s>0, \forall \varepsilon \in\left(0, \varepsilon_{1}\right], \forall I \in K .
$$

We now estimate $A_{2, \varepsilon}$ as follows.

Using the exponential decay of the functions $\frac{\partial \hat{\psi}_{i, \varepsilon}}{\partial \tau}(., I)$ at $\pm \infty$, since (2.64) holds, we have

$$
\begin{aligned}
& \quad\left\|A_{2, \varepsilon}(., I)\right\|_{0,1, \Omega} \\
& \leq \varepsilon^{-1 / 2} \sum_{i \in Q \cup S_{l}}\left\|M_{i, \Delta}\right\|_{0, \infty, \Omega} \\
& \quad \times\left\|\frac{\partial \hat{\psi}_{i, \varepsilon}}{\partial \tau}\left(\tau_{i, \varepsilon}(.), I\right)\left(n\left(a_{i}^{\nu}, I\right)-n(., I)\right)\right\|_{0,1,\left[a_{i}-\Delta, a_{i}+\Delta\right] \cap \Omega} \\
& \quad+C \varepsilon^{-1 / 2} \sum_{i \in P}\left\|M_{i, \Delta}\right\|_{0, \infty, \Omega}\left\|\frac{\partial \hat{\psi}_{i, \varepsilon}}{\partial \tau}\left(\tau_{i, \varepsilon}(.), I\right)\right\|_{0,1,\left[a_{i}-\Delta, a_{i}+\Delta\right] \cap \Omega} \\
& +C_{s} \varepsilon^{s} \quad \forall s>0, \forall \varepsilon \in\left(0, \varepsilon_{1}\right], \forall I \in K .
\end{aligned}
$$

We now remark that we have

$$
\begin{aligned}
& \varepsilon^{-1 / 2}\left\|\frac{\partial \hat{\psi}_{i, \varepsilon}}{\partial \tau}\left(\tau_{i, \varepsilon}(.), I\right)\left(n\left(a_{i}^{\nu}, I\right)-n(., I)\right)\right\|_{0,1,\left[a_{i}-\Delta, a_{i}+\Delta\right] \cap \Omega} \\
& \leq \varepsilon^{-1 / 2}|n(., I)|_{1, \infty, \Omega, \mathcal{P}_{M}}\left\|\frac{\partial \hat{\psi}_{i, \varepsilon}}{\partial \tau}\left(\tau_{i, \varepsilon}(.), I\right)\left(. \quad-a_{i}\right)\right\|_{0,1,\left[a_{i}-\Delta, a_{i}+\Delta\right] \cap \Omega}, \quad \forall i \in Q \cup S_{l} .
\end{aligned}
$$

Making the change of variable $\tau_{j, \varepsilon}=\tau_{j, \varepsilon}(x)$ in this last expression, we obtain

$$
\begin{array}{r}
\varepsilon^{-1 / 2}\left\|\frac{\partial \hat{\psi}_{i, \varepsilon}}{\partial \tau}\left(\tau_{i, \varepsilon}(.), I\right)\left(n\left(a_{i}^{\nu}, I\right)-n(., I)\right)\right\|_{0,1,\left[a_{i}-\Delta, a_{i}+\Delta\right] \cap \Omega} \leq C \sqrt{\varepsilon} \\
\forall \varepsilon \in\left(0, \varepsilon_{1}\right], \forall I \in K, \forall i \in Q \cup S_{l} .
\end{array}
$$

Using now the definition (2.61) of $\hat{\psi}_{i, \varepsilon}$ for $i \in P$, we easily obtain

$$
\begin{array}{r}
\varepsilon^{-1 / 2} \sum_{i \in P}\left\|M_{i, \Delta}\right\|_{0, \infty, \Omega}\left\|\frac{\partial \hat{\psi}_{i, \varepsilon}}{\partial \tau}\left(\tau_{i, \varepsilon}(.), I\right)\right\|_{0,1,\left[a_{i}-\Delta, a_{i}+\Delta\right] \cap \Omega} \leq C \sqrt{\varepsilon} \\
\forall \varepsilon \in\left(0, \varepsilon_{1}\right], \quad \forall I \in K .
\end{array}
$$

Using these last two estimates in (4.46), we obtain

$$
\left\|A_{2, \varepsilon}(., I)\right\|_{0,1, \Omega} \leq C \sqrt{\varepsilon} \quad \forall \varepsilon \in\left(0, \varepsilon_{1}\right], \forall I \in K .
$$

Hence we have proved that

$$
\left\|R_{2, \varepsilon}(., I)\right\|_{0,1, \Omega} \leq C \sqrt{\varepsilon} \quad \forall \varepsilon \in\left(0, \varepsilon_{1}\right], \forall I \in K .
$$

In a similar way we prove that

$$
\left\|R_{3, \varepsilon}(., I)\right\|_{0,1, \Omega} \leq C \sqrt{\varepsilon} \quad \forall \varepsilon \in\left(0, \varepsilon_{1}\right], \forall I \in K .
$$

Hence we have

$$
\left\|R_{\varepsilon}(., I)\right\|_{Y} \leq C \sqrt{\varepsilon} \quad \forall \varepsilon \in\left(0, \varepsilon_{1}\right], \forall I \in K .
$$


Finally, thanks to the exponential decay of the functions $\frac{\partial^{s+1} \hat{\psi}_{i, \varepsilon}}{\partial \tau^{s} \partial I}(., I)$ at $\pm \infty$ for $s=0,1$, and using the above techniques, we deduce that

$$
\left\|\frac{\partial R_{\varepsilon}}{\partial I}(., I)\right\|_{Y} \leq C \sqrt{\varepsilon} \quad \forall \varepsilon \in\left(0, \varepsilon_{1}\right], \forall I \in K .
$$

We are now able to prove theorem 3.3.

Proof of theorem 3.3. We first remark that $\left(\tilde{\psi}_{\varepsilon}, n_{\varepsilon}, p_{\varepsilon}\right)$ satisfies (3.1)-(3.2), where $r_{-}=r_{-, \varepsilon}=\min \left(\min _{\bar{\Omega}}\left(n_{\varepsilon}\right), \min _{\bar{\Omega}}\left(p_{\varepsilon}\right)\right)>0$. Moreover, using $(2.66)$ we have

$$
\begin{aligned}
& \left\|\tilde{\psi}_{\varepsilon}^{\prime}(., I)\right\|_{0,1, \Omega}+\left\|n_{\varepsilon}(., I)\right\|_{0, \infty, \Omega} \\
& +\left\|p_{\varepsilon}(., I)\right\|_{0, \infty, \Omega} \leq C \quad \forall \varepsilon \in\left(0, \varepsilon_{1}\right], \quad \forall I \in K .
\end{aligned}
$$

Using lemma 4.2 and 4.3 (with $q=0$ ), together with theorem 3.1, we first deduce that

$$
\left\|u_{i, \varepsilon}(., I)\right\|_{0, \infty, \Omega} \leq C \sqrt{\varepsilon} \quad \forall \varepsilon \in\left(0, \varepsilon_{1}\right], \forall I \in K, i=2,3 .
$$

Since $u_{2, \varepsilon}=n_{\varepsilon}-\tilde{n}_{\varepsilon}, u_{3, \varepsilon}=p_{\varepsilon}-\tilde{p}_{\varepsilon}$, and using the inequality (2.69) of lemma 2.6, we deduce from (4.48) that there exists $r_{-}=r_{-}\left(K, I_{0}, N, \delta, \mathcal{P}_{M}\right)>0$ independent of $\varepsilon$ such that

$$
n_{\varepsilon}(x, I) \geq r_{-}, \quad p_{\varepsilon}(x, I) \geq r_{-} \quad \forall x \in \bar{\Omega}, \forall I \in K, \forall \varepsilon \in\left(0, \varepsilon_{1}\right] .
$$

Hence $\left(n_{\varepsilon}, p_{\varepsilon}\right)$ satisfies (3.2) with $r_{-}>0$ independent of $\varepsilon$. Using once again theorem 3.1, together with lemmas 4.2 and 4.3 (with $q=0$ ), since $r_{-}$is independent o $\varepsilon$ we deduce that (3.12) holds for $q=0$.

Since

$$
U_{\varepsilon}=\left(\psi_{\varepsilon}-\tilde{\psi}_{\varepsilon}, n_{\varepsilon}-\tilde{n}_{\varepsilon}, p_{\varepsilon}-\tilde{p}_{\varepsilon}, J_{n, \varepsilon}-J_{n}\right)
$$

satisfies (4.34) and is smooth with respect to $I \in K$, we deduce that $\frac{\partial U_{\varepsilon}}{\partial I}$ satisfies the equation

$$
A_{\left(\tilde{\psi}_{\varepsilon}, n_{\varepsilon}, p_{\varepsilon}\right)}^{\varepsilon}\left(\frac{\partial U_{\varepsilon}}{\partial I}\right)=\frac{\partial R_{\varepsilon}}{\partial I}+\bar{R}_{\varepsilon},
$$

where $\bar{R}_{\varepsilon}=\left(\bar{R}_{i, \varepsilon}(., I)\right)_{1 \leq i \leq 9} \in Y$ is given by

$$
\begin{gathered}
\bar{R}_{1, \varepsilon}(., I)=\bar{R}_{4, \varepsilon}(., I)=\bar{R}_{i, \varepsilon}=0 \quad \forall i \in\{5, \ldots, 9\}, \\
\bar{R}_{2, \varepsilon}(., I)=\left(n_{\varepsilon}-\tilde{n}_{\varepsilon}\right)(., I) \frac{\partial^{2} \tilde{\psi}_{\varepsilon}}{\partial x \partial I}(., I)+\frac{\partial n_{\varepsilon}}{\partial I}(., I) \frac{\partial\left(\psi_{\varepsilon}-\tilde{\psi}_{\varepsilon}\right)}{\partial x}(., I), \\
\bar{R}_{3, \varepsilon}(., I)=-\left(p_{\varepsilon}-\tilde{p}_{\varepsilon}\right)(., I) \frac{\partial^{2} \tilde{\psi}_{\varepsilon}}{\partial x \partial I}(., I)-\frac{\partial p_{\varepsilon}}{\partial I}(., I) \frac{\partial\left(\psi_{\varepsilon}-\tilde{\psi}_{\varepsilon}\right)}{\partial x}(., I) .
\end{gathered}
$$

Hence we have

$$
\begin{aligned}
& \left\|\left.\bar{R}_{2, \varepsilon}(., I)\right|_{0,1, \Omega} \leq\right\|\left(n_{\varepsilon}-\tilde{n}_{\varepsilon}\right)(., I)\left\|_{0, \infty, \Omega}\right\| \frac{\partial^{2} \tilde{\psi}_{\varepsilon}}{\partial x \partial I}(., I) \|_{0,1, \Omega} \\
& +\left\|\frac{\partial n_{\varepsilon}}{\partial I}(., I)\right\|_{0, \infty, \Omega}\left\|\left(\psi_{\varepsilon}-\tilde{\psi}_{\varepsilon}\right)(., I)\right\|_{1,1, \Omega} .
\end{aligned}
$$


We first estimate $\left\|\frac{\partial n_{\varepsilon}}{\partial I}(., I)\right\|_{0, \infty, \Omega}$ as follows. Since $\left(\psi_{\varepsilon}, n_{\varepsilon}, p_{\varepsilon}, J_{n, \varepsilon}\right)(., I)$ is the solution of $(\mathrm{CD})_{I, \varepsilon}$ we deduce that

$$
W_{\varepsilon}=\left(\frac{\partial \psi_{\varepsilon}}{\partial I}, \frac{\partial n_{\varepsilon}}{\partial I}, \frac{\partial p_{\varepsilon}}{\partial I}, \frac{\partial J_{n, \varepsilon}}{\partial I}\right) \in X
$$

satisfies the equation

$$
A_{\left(\psi_{\varepsilon}, n_{\varepsilon}, p_{\varepsilon}\right)}^{\varepsilon} W_{\varepsilon}=\tilde{R}_{\varepsilon} \in Y
$$

where $A_{\left(\psi_{\varepsilon}, n_{\varepsilon}, p_{\varepsilon}\right)}^{\varepsilon}$ is the operator defined by (3.3) and $\tilde{R}_{\varepsilon}(., I)=\left(\tilde{R}_{i, \varepsilon}(., I)\right)_{1 \leq i \leq 9}$ is given by $\tilde{R}_{i, \varepsilon}=0$ for $i \in\{1, \ldots, 9\}-\{3\}$ and $\tilde{R}_{3, \varepsilon}=-1$. We first remark that $\left(\psi_{\varepsilon}, n_{\varepsilon}, p_{\varepsilon}\right)$ satisfies (3.1)-(3.2), where $r_{-}$is independent of $\varepsilon$. Moreover, since (3.12) holds for $q=0$ and since $\left\|\tilde{\psi}_{\varepsilon}^{\prime}(., I)\right\|_{0,1, \Omega} \leq C$ for all $\varepsilon \in\left(0, \varepsilon_{1}\right]$ and all $I \in K$, we deduce that $\left\|\psi_{\varepsilon}^{\prime}(., I)\right\|_{0,1, \Omega} \leq C$ for all $\varepsilon \in\left(0, \varepsilon_{1}\right]$ and all $I \in K$. Hence we deduce from the inequalities (3.5)-(3.6) of theorem 3.1 that

$$
\begin{array}{r}
\left\|\frac{\partial \psi_{\varepsilon}}{\partial I}(., I)\right\|_{1,1, \Omega}+\left\|\frac{\partial n_{\varepsilon}}{\partial I}(., I)\right\|_{0, \infty, \Omega}+ \\
\qquad \frac{\partial p_{\varepsilon}}{\partial I}(., I) \|_{0, \infty, \Omega} \leq C \\
\forall \varepsilon \in\left(0, \varepsilon_{1}\right], \forall I \in K .
\end{array}
$$

Using this last estimate in (4.50) together with (3.12) for $q=0$, we deduce that

$$
\|\left.\bar{R}_{2, \varepsilon}(., I)\right|_{0,1, \Omega} \leq C \sqrt{\varepsilon} \quad \forall \varepsilon \in\left(0, \varepsilon_{1}\right], \forall I \in K .
$$

We prove in a similar way that

$$
\|\left.\bar{R}_{3, \varepsilon}(., I)\right|_{0,1, \Omega} \leq C \sqrt{\varepsilon} \quad \forall \varepsilon \in\left(0, \varepsilon_{1}\right], \forall I \in K .
$$

Using theorem 3.1 once again, together with the estimate (4.45) of lemma 4.3 (with $q=1$ ) and the above estimates on $\bar{R}_{i, \varepsilon}$ for $i=2,3$, we obtain (3.12) for $q=1$.

4.3. Proof of the multiplicity results. We are now able to prove theorem 3.4 and corollary 3.5.

Proof of theorem 3.4. Assume first that there exists $V_{0}>0$ such that

$$
\#\left(\mathcal{S}\left(V_{0}\right) \cap \mathcal{S}_{n c}\right)=k \geq 2
$$

and denote by $I_{1}<\ldots<I_{k}$ the elements of $\mathcal{S}\left(V_{0}\right) \cap \mathcal{S}_{n c}$. We choose $\eta>0$ as in property (ii) of corollary 2.3 , and for $I \in \mathbb{R}$ let $\left(\psi_{\varepsilon}, n_{\varepsilon}, p_{\varepsilon}, J_{n, \varepsilon}\right)(., I)$ be the solution of $(\mathrm{CD})_{I, \varepsilon}$. Since $K=\bigcup_{1 \leq j \leq k}\left[I_{j}-\eta, I_{j}+\eta\right] \subset \mathcal{E}\left(N, \delta, \mathcal{P}_{M}\right)$, we can define $\left(\tilde{\psi}_{\varepsilon}, \tilde{n}_{\varepsilon}, \tilde{p}_{\varepsilon}, J_{n}\right)(., I)$ as in (2.66)-(2.68) for all $I \in K$.

Thanks to theorem 3.3 we deduce that there exists $\varepsilon_{1}=\varepsilon_{1}\left(N, \delta, \mathcal{P}_{M}\right)>0$ such that

$$
\left|\psi_{\varepsilon}(1, I)-\tilde{\psi}_{\varepsilon}(1, I)\right|+\left|\frac{\partial \psi_{\varepsilon}}{\partial I}(1, I)-\frac{\partial \tilde{\psi}_{\varepsilon}}{\partial I}(1, I)\right| \leq C \sqrt{\varepsilon} \quad \forall \varepsilon \in\left(0, \varepsilon_{1}\right), \forall I \in K .
$$

Using (1.10) we have

$$
\psi_{\varepsilon}(1, I)=\log \left(\frac{n_{1}}{n_{-1}}\right)+\psi_{-1}--\mathcal{V}_{\varepsilon}(I), \quad \forall I \in \mathbb{R} .
$$


Moreover, thanks to (2.10) and (2.19) we have

$$
\tilde{\psi}_{\varepsilon}(1, I)=\psi(1, I)+\hat{\psi}_{M+1, \varepsilon}\left(0^{-}, I\right)=\log \left(\frac{n_{1}}{n_{r,-1}}\right)+\psi_{r,-1}-\mathcal{V}(I), \quad \forall I \in K .
$$

Using (4.53)-(4.54) and (2.9) in (4.52), we obtain

$$
\left|\mathcal{V}_{\varepsilon}(I)-\mathcal{V}(I)\right|+\left|\mathcal{V}_{\varepsilon}^{\prime}(I)-\mathcal{V}^{\prime}(I)\right| \leq C \sqrt{\varepsilon} \quad \varepsilon \in\left(0, \varepsilon_{1}\right), \quad \forall I \in K .
$$

Using now property (ii) of corollary 2.3 together with (4.55), we deduce that for $\varepsilon$ sufficiently small we have

$$
\begin{aligned}
& \mathcal{V}_{\varepsilon}^{\prime}<0 \quad(\text { resp. }>0) \quad \text { on }\left[I_{j}-\eta, I_{j}+\eta\right] \\
& \forall j \in\{2,4, \ldots, k-1\} \quad(\text { resp. }\{1,3, \ldots, k\}) .
\end{aligned}
$$

We set $\gamma_{j}=1$ (resp. -1 ) if $j \in\{2,4, \ldots, k-1\}$ (resp. $\{1,3, \ldots, \mathrm{k}\}$ ). Then using (4.55) once again we obtain

$$
\begin{aligned}
& \mathcal{V}_{\varepsilon}\left(I_{j}+\gamma_{j} \eta\right) \leq V_{0}+\left(\mathcal{V}\left(I_{j}+\gamma_{j} \eta\right)-\mathcal{V}\left(I_{j}\right)+C \sqrt{\varepsilon}\right) \\
& \mathcal{V}_{\varepsilon}\left(I_{j}-\gamma_{j} \eta\right) \geq V_{0}+\left(\mathcal{V}\left(I_{j}-\gamma_{j} \eta\right)-\mathcal{V}\left(I_{j}\right)-C \sqrt{\varepsilon}\right)
\end{aligned}
$$

Since property (ii) of corollary 2.3 holds, we see that there exists $\varepsilon_{2}=\varepsilon_{2}\left(N, \delta, \mathcal{P}_{M}\right)$ $\in\left(0, \varepsilon_{1}\right)$ such that for all $\varepsilon \in\left(0, \varepsilon_{2}\right),(4.56)$ and

$$
\begin{aligned}
& \mathcal{V}_{\varepsilon}\left(I_{j}+\eta\right)<V_{0}<\mathcal{V}_{\varepsilon}\left(I_{j}-\eta\right) \quad\left(\operatorname{resp} . \mathcal{V}_{\varepsilon}\left(I_{j}-\eta\right)<V_{0}<\mathcal{V}_{\varepsilon}\left(I_{j}+\eta\right)\right) \\
& \forall j \in\{2,4, \ldots, k-1\} \quad(\text { resp. }\{1,3, \ldots, k\})
\end{aligned}
$$

hold. We then easily conclude our argument. The proof is similar if $V_{0}<0$.

Proof of corollary 3.5. Assume first that $\nu=+$. Since $0 \in \mathcal{E}\left(N, \delta, \mathcal{P}_{M}\right)$ and since $\mathcal{C}_{\text {sat }, l}\left(N, \delta, \mathcal{P}_{M}\right) \cap R^{\nu} \neq \emptyset$, we deduce that $0<J_{1}=\sup \left(\mathcal{O}\left(0, N, \delta, \mathcal{P}_{M}\right)\right)<+\infty$ and $J_{1} \in \mathcal{C}_{\text {sat }, l}\left(N, \delta, \mathcal{P}_{M}\right) \cap R^{\nu}$. Of course $\left[0, J_{1}\right) \subset \mathcal{E}\left(N, \delta, \mathcal{P}_{M}\right)$. Moreover, thanks to our assumptions there exists $J_{2} \in \mathcal{C}_{s a t, r}\left(N, \delta, \mathcal{P}_{M}\right) \cap R^{\nu}$. Of course we have $J_{2} \geq J_{1}$. Moreover, by definition of $\mathcal{C}_{\text {sat }, r}\left(N, \delta, \mathcal{P}_{M}\right)$, there exists $\gamma>0$ such that $\left(J_{2}, J_{2}+\gamma\right] \subset \mathcal{E}\left(N, \delta, \mathcal{P}_{M}\right)$. We set $J_{3}=\sup \left(\mathcal{O}\left(J_{2}+\gamma, N, \delta, \mathcal{P}_{M}\right)\right)$; then $J_{3} \in\left(J_{2},+\infty\right]$ and $\left(J_{2}, J_{3}\right) \subset \mathcal{E}\left(N, \delta, \mathcal{P}_{M}\right)$. Thanks to property (iv) of theorem 2.2 we have

$$
\lim _{I \rightarrow J_{m}^{-}} \mathcal{V}(I)=+\infty, m \in\{1,3\}, \quad \lim _{I \rightarrow J_{2}^{+}} \mathcal{V}(I)=+\infty
$$

Hence there exists $J^{\star} \in\left(J_{2}, J_{3}\right)$ such that $\inf _{I \in\left(J_{2}, J_{3}\right)} \mathcal{V}(I)=\mathcal{V}\left(J^{\star}\right)>0$. Since $\mathcal{V}$ is analytic on $\mathcal{E}\left(N, \delta, \mathcal{P}_{M}\right)$, we deduce that there exist $I_{1} \in\left(J_{2}, J^{\star}\right)$ and $I_{2} \in\left(J^{\star}, J_{3}\right)$ such that $\mathcal{V}^{\prime}\left(I_{1}\right)<0, \mathcal{V}^{\prime}\left(I_{2}\right)>0$ and $\mathcal{V}\left(I_{1}\right)=\mathcal{V}\left(I_{2}\right)=V_{0}>\mathcal{V}\left(J^{\star}\right)$. Therefore $\#\left(\mathcal{S}\left(V_{0}\right) \cap \mathcal{S}_{n c}\right)=k \geq 2$. From corollary 2.3 we know that $k \geq 3$. We then conclude by applying theorem 3.4. The proof is similar if $\nu=-$.

\section{Part II. the electroneutral case: a constructive method for multiplicity}

Part II will be devoted to the analysis of the roles of $N, \delta$ and $V$ on the existence of multiple solutions of $(\mathrm{RVD})_{V}$. 


\section{A SUfFICIENT CONDITION FOR THE EXISTENCE OF SATURATiON CURRENT}

We consider for the rest of this paper the reduced current driven equations $(\mathrm{RCD})_{I}$. In order to shorten certain computations it is useful to consider $\Omega=(0,1)$ instead of $(-1,1)$. Hence we replace -1 by 0 in $(\mathrm{RCD})_{I}$ and in $(2.10)$ and whenever else it is necessary, without further specifying it.

Assume that $I \in \mathcal{E}\left(N, \delta, \mathcal{P}_{M}\right)$ and that $\left(\psi, n, p, J_{n}\right)$ is the solution of $(\mathrm{RCD})_{I}$. Then we deduce easily that

$$
n(x)>0, p(x) \quad \forall x \in \bar{\Omega},
$$

and that

$$
n=\frac{N+s}{2}, p=\frac{-N+s}{2},
$$

where

$$
s=n+p
$$

Hence thanks to (5.1) we have

$$
s(x)>|N(x)| \quad \forall x \in \bar{\Omega} .
$$

Using the equations of $(\mathrm{RCD})_{I}$, we also deduce that $s$ and

$$
J=J_{n}-\frac{I}{2}
$$

satisfy

$$
\begin{gathered}
s^{\prime}=\frac{N\left(N^{\prime}-I\right)}{s}+2 J \quad \text { in } \Omega_{M}, \\
s(x)=n_{r, x}+p_{r, x}, \quad x \in\{0,1\}, \\
{\left[s^{2}-N^{2}\right]_{a_{i}}=0 \quad \forall i \in\{1, \ldots, M\} .}
\end{gathered}
$$

Moreover $\psi$ satisfies

$$
\begin{gathered}
\psi^{\prime}=\frac{N^{\prime}-I}{s} \quad \text { in } \Omega_{M}, \\
\psi(0)=\psi_{r, 0} \\
{[\psi]_{a_{i}}=\log \left(\frac{N\left(a_{i}^{+}\right)+s\left(a_{i}^{+}\right)}{N\left(a_{i}^{-}\right)+s\left(a_{i}^{-}\right)}\right) \quad \forall i \in\{1, \ldots, M\} .}
\end{gathered}
$$

Conversely if $(s, J)$ satisfies (5.4)-(5.8) and $\psi$ satisfies (5.9)-(5.11), then $\left(\psi, n, p, J_{n}\right)$ is the solution of $(\mathrm{RCD})_{I}$, where $n$ and $p$ are given by (5.2) and where

$$
J_{n}=J+\frac{I}{2} .
$$

Hence we have proved the following result.

Lemma 5.1. Assume that $N \in W^{1, \infty}\left(\Omega, \mathcal{P}_{M}\right)$. Then for all $I \in \mathcal{E}\left(N, \delta, \mathcal{P}_{M}\right)$, $\left(\psi, n, p, J_{n}\right)$ is the solution of $(R C D)_{I}$ if and only if $(s, J)$ and $\psi$ satisfy respectively (5.4)-(5.8) and (5.9)-(5.11), where $(s, J)$ are respectively given by (5.3) and (5.5). Moreover if $(s, J)$ is a solution of (5.4)-(5.8) and $\psi$ a solution of (5.9)-(5.11), then $\left(\psi, n, p, J_{n}\right)$ is the solution of $(R C D)_{I}$, where $n$ and $p$ are given by (5.2) and $J_{n}$ by (5.12). 
We set

$$
s_{r, x}=n_{r, x}+p_{r, x}, \quad x \in\{0,1\} .
$$

For $N \in W^{1, \infty}\left(\Omega, \mathcal{P}_{M}\right)$ we define

$$
g_{0, N}(x)=\int_{0}^{x} N(t) d t, \quad g_{1, N}(x)=\int_{x}^{1} N(t) d t .
$$

Then we have the following result, whose proof is easy and is left to the reader.

Proposition 5.2. Assume that $N \in W^{1, \infty}\left(\Omega, \mathcal{P}_{M}\right)$ and denote by $\left(\psi, n, p, J_{n}\right)$ the solution of $(R C D)_{I}$ for $I \in \mathcal{E}\left(N, \delta, \mathcal{P}_{M}\right)$. Then $(s, J)=\left(n+p, J_{n}-I / 2\right)$ satisfies

$$
\begin{gathered}
\left(s^{2}-N^{2}\right)^{\prime}(x)=-2 I N+4 J s \quad \text { in } \Omega, \\
\left(s^{2}-N^{2}\right)(x)=4 \delta^{4}, \quad x \in\{0,1\} .
\end{gathered}
$$

We deduce in particular that $J$ satisfies

$$
J=\frac{I \int_{0}^{1} N d t}{2 \int_{0}^{1} s d t} .
$$

Moreover, if $\operatorname{Ig}_{0, N}(1) \leq 0$ (resp. $\left.\geq 0\right)$ then

$$
\begin{aligned}
& \left(s^{2}-N^{2}\right)(x) \leq 4 \delta^{4}-2 I g_{0, N}(x) \\
& \left(\text { resp. } \quad\left(s^{2}-N^{2}\right)(x) \leq 4 \delta^{4}+2 I g_{1, N}(x)\right) \quad \forall x \in \Omega,
\end{aligned}
$$

where $g_{0, N}$ and $g_{1, N}$ are defined as in (5.14).

We now give a sufficient condition on the data for the non-existence of a branch of solution of $(\mathrm{RCD})_{I}$ at infinity.

Lemma 5.3. Assume that $N \in W^{1, \infty}\left(\Omega, \mathcal{P}_{M}\right)$. Then the following properties hold.

(i): Assume that $g_{0, N}(1)<0$. If $\max _{\Omega} g_{0, N}>0\left(\right.$ resp. $\left.\max _{\Omega} g_{1, N}>0\right),(R C D)_{I}$ has no solutions for

$$
I \geq 2 \delta^{4}\left(\max _{\Omega} g_{0, N}\right)^{-1} \quad\left(\text { resp. } I \leq-2 \delta^{4}\left(\max _{\Omega} g_{1, N}\right)^{-1}\right) .
$$

(ii): Assume that $g_{0, N}(1)>0$. If $\min _{\Omega} g_{1, N}<0$ (resp. $\left.\min _{\Omega} g_{0, N}<0\right),(R C D)_{I}$ has no solutions for

$$
I \geq-2 \delta^{4}\left(\min _{\Omega} g_{1, N}\right)^{-1} \quad\left(\text { resp. } I \leq 2 \delta^{4}\left(\min _{\Omega} g_{0, N}\right)^{-1}\right) .
$$

(iii): Assume that $g_{0, N}(1)=0$. Then $\mathcal{E}\left(N, \delta, \mathcal{P}_{M}\right)=\left(a_{-}(N, \delta), a_{+}(N, \delta)\right)$, where

$$
a_{-}(N, \delta)=2 \delta^{4}\left(\min _{\Omega} g_{0, N}\right)^{-1} \quad(\text { resp. }-\infty) \text { if } \min _{\Omega} g_{0, N}<0(\text { resp. } \geq 0),
$$

and

$$
a_{+}(N, \delta)=2 \delta^{4}\left(\max _{\Omega} g_{0, N}\right)^{-1} \quad(\text { resp. }+\infty) \text { if } \max _{\Omega} g_{0, N}>0(\text { resp. } \leq 0) .
$$


Proof. Let $I \in \mathcal{E}\left(N, \delta, \mathcal{P}_{M}\right)$ be given. From proposition 5.2 we know that (5.18) holds. Assume first that $g_{0, N}(1) \leq 0$ and $I \geq 0$. Then from (5.18) and (5.4) we deduce that if $\max _{\Omega} g_{0, N}>0$ then

$$
0 \leq I<2 \delta^{4}\left(\max _{\Omega} g_{0, N}>0\right)^{-1},
$$

so that $\sup \left(\mathcal{E}\left(N, \delta, \mathcal{P}_{M}\right)\right) \leq 2 \delta^{4}\left(\max _{\Omega} g_{0, N}>0\right)^{-1}$. In a similar way we deduce that if $g_{0, N}(1) \geq 0$ and $I \leq 0$ and $\max _{\Omega} g_{1, N}>0$ then

$$
\inf \left(\mathcal{E}\left(N, \delta, \mathcal{P}_{M}\right)\right) \geq-2 \delta^{4}\left(\max _{\Omega} g_{1, N}>0\right)^{-1} .
$$

This proves (i). The case (ii) can be treated in a similar way.

Finally if $g_{0, N}(1)=0$, then

$$
\left(s^{2}-N^{2}\right)=4 \delta^{4}-2 I g_{0, N},
$$

so that we easily obtain (iii).

In order to apply the multiplicity results of section 3 we want to give examples of data for which the reduced current driven equations have at least two saturation currents. Of course under the conditions of the previous lemma we proved the existence of at least one saturation current. In order to obtain the existence of a second saturation current we must guarantee that the solutions start to exist again beyond the first saturation current. As will be seen in the sequel, this will be done by analyzing $(\mathrm{RCD})_{I}$ at infinity. Hence we must find conditions sharper than the one given in the previous lemma for the existence of a saturation current without losing the existence of a branch of solutions at infinity. This is done in the next result.

Theorem 5.4. Assume that $N \in W^{1, \infty}\left(\Omega, \mathcal{P}_{M}\right)$ and define

$$
F(x, N)=\int_{x}^{1} N(t) d t \int_{0}^{1}|N(t)| d t-\int_{0}^{1} N(t) d t \int_{x}^{1}|N(t)| d t
$$

Then the following properties hold:

If $\max _{\Omega} g_{0, N} \leq 0$ or $\min _{\Omega} g_{1, N} \geq 0$, then if $\min _{\Omega} F(x, N)<0$, there exists $\delta_{0}>0$ such that for every $0<\delta<\delta_{0}(R C D)_{I}$ has at least one positive saturation current.

If $\max _{\Omega} g_{1, N} \leq 0$ or $\min _{\Omega} g_{0, N} \geq 0$, then if $\max _{\Omega} F(x, N)>0$, there exists $\delta_{0}>0$ such that for every $0<\delta<\delta_{0}(R C D)_{I}$ has at least one negative saturation current.

Proof. Let $I \in \mathcal{E}\left(N, \delta, \mathcal{P}_{M}\right)$ be given.

Case (1). Assume first that $\max _{\Omega} g_{0, N} \leq 0$ (resp. $\min _{\Omega} g_{0, N} \geq 0$ ) if $I \geq 0$ (resp. $I \leq 0$ ). Then we deduce from proposition 5.2 (see (5.18)) together with (5.4) that

$$
|N| \leq s \leq h_{0} \text { on } \bar{\Omega},
$$

where

$$
h_{0}=\sqrt{N^{2}+4 \delta^{4}}+\sqrt{2|I|} \sqrt{\left|g_{0, N}\right|} .
$$

Moreover in both cases, since $I g_{0, N}(1) \leq 0$, we have

$$
\frac{I \int_{0}^{1} N(t) d t}{2 \int_{0}^{1}|N(t)| d t} \leq J \leq 0 .
$$


This, together with $h_{0} \geq 0$, yields

$$
\frac{I h_{0} \int_{0}^{1} N(t) d t}{2 \int_{0}^{1}|N(t)| d t} \leq J h_{0} \leq J s
$$

Inserting this last inequality in (5.15), integrating the resulting inequality from $x$ to 1 and using (5.16), we obtain

$$
\left(s^{2}-N^{2}\right)(x) \leq P_{0}(x, \delta, N, \sqrt{|I|}) \quad \forall x \in \bar{\Omega},
$$

where

$$
P_{0}(x, \delta, N, \lambda)=4 \delta^{4}+2 \lambda^{2} \alpha_{0}(x, \delta, N)+\lambda^{3} \beta_{0}(x, N),
$$

where

$$
\alpha_{0}(x, \delta, N)=\operatorname{sign}(I)\left(\int_{x}^{1} N(t) d t-\frac{\int_{0}^{1} N(t) d t \int_{x}^{1} \sqrt{N^{2}+4 \delta^{4}} d t}{\int_{0}^{1}|N(t)| d t}\right),
$$

and

$$
\beta_{0}(x, N)=-2 \sqrt{2} \operatorname{sign}(I)\left(\frac{\int_{0}^{1} N(t) d t \int_{x}^{1} \sqrt{\left|g_{0, N}\right|} d t}{\int_{0}^{1}|N(t)| d t}\right)
$$

Under case (1) we have $\beta_{0}(., N) \geq 0$. Assume now that $\min _{\Omega} F(x, N)<0$. Then there exists $x_{0} \in \bar{\Omega}$ such that $\alpha_{0}\left(x_{0}, 0, N\right)<0$. We now remark that if $\alpha_{0}(x, \delta, N)<0$ then $P_{0}(x, \delta, N, \lambda)$ has a minimum with respect to $\lambda$ on $\mathbb{R}^{+}$at $\lambda=-4 \alpha_{0}(x, \delta, N)\left(3 \beta_{0}(x, N)\right)^{-1}$. This minimum is nonpositive if and only if

$$
\alpha_{0}(x, \delta, N)+\frac{3}{2} \delta^{4 / 3}\left(\beta_{0}(x, N)\right)^{2 / 3} \leq 0 .
$$

Hence, since the left hand side of (5.26) is continuous with respect to $\delta$, we deduce that there exists $\delta_{0}>0$ such that (5.26) holds for $x=x_{0}$ and $0 \leq \delta \leq \delta_{0}$. But then there exist $0<\lambda_{-}<\lambda_{+}$such that $P_{0}\left(x_{0}, \delta, N, \lambda\right) \leq 0$ for every $\lambda \in\left[\lambda_{-}, \lambda_{+}\right]$. But this, together with (5.22), contradicts (5.4) (see lemma 5.1), which proves that $(\mathrm{RCD})_{I}$ has no solutions for $I \in\left[\lambda_{-}^{2}, \lambda_{+}^{2}\right]$. Hence $(\mathrm{RCD})_{I}$ has necessarily one positive saturation current.

Case (2): Assume now that $\min _{\Omega} g_{1, N} \geq 0$ (resp. $\left.\max _{\Omega} g_{1, N} \leq 0\right)$ if $I \geq 0$ (resp. $I \leq 0$ ). Then we deduce from proposition 5.2 (see (5.18)) together with (5.4) that

$$
|N| \leq s \leq h_{1} \text { on } \bar{\Omega}
$$

where

$$
h_{1}=\sqrt{N^{2}+4 \delta^{4}}+\sqrt{2|I|} \sqrt{\left|g_{1, N}\right|} .
$$

Moreover in both cases, since $I g_{0, N}(1) \geq 0$, we have

$$
\frac{I \int_{0}^{1} N(t) d t}{2 \int_{0}^{1}|N(t)| d t} \geq J \geq 0 .
$$

This, together with $h_{1} \geq 0$, yields

$$
\frac{I h_{1} \int_{0}^{1} N(t) d t}{2 \int_{0}^{1}|N(t)| d t} \geq J h_{1} \geq J s
$$


Inserting this last inequality in (5.15), integrating the resulting inequality from $x$ to 1 and using (5.16), we obtain

$$
\left(s^{2}-N^{2}\right)(x) \leq P_{1}(x, \delta, N, \sqrt{|I|}) \quad \forall x \in \bar{\Omega},
$$

where

$$
P_{1}(x, \delta, N, \lambda)=4 \delta^{4}+2 \lambda^{2} \alpha_{1}(x, \delta, N)+\lambda^{3} \beta_{1}(x, N),
$$

where

$$
\alpha_{1}(x, \delta, N)=\operatorname{sign}(I)\left(-\int_{0}^{x} N(t) d t+\frac{\int_{0}^{1} N(t) d t \int_{0}^{x} \sqrt{N^{2}+4 \delta^{4}} d t}{\int_{0}^{1}|N(t)| d t}\right),
$$

and

$$
\beta_{1}(x, N)=2 \sqrt{2} \operatorname{sign}(I)\left(\frac{\int_{0}^{1} N(t) d t \int_{0}^{x} \sqrt{\left|g_{1, N}\right|} d t}{\int_{0}^{1}|N(t)| d t}\right) .
$$

Remarking now that

$$
\begin{aligned}
& -\int_{0}^{x} N(t) d t \int_{0}^{1}|N(t)| d t+\int_{0}^{1} N(t) d t \int_{0}^{x}|N(t)| d t \\
& =\int_{x}^{1} N(t) d t \int_{0}^{1}|N(t)| d t-\int_{0}^{1} N(t) d t \int_{x}^{1}|N(t)| d t
\end{aligned}
$$

we conclude as for case (1).

\section{Sufficient CONDitions For the EXISTEnCE OF A BRANCH OF SOLUTIONS AT INFINITY}

The purpose of the next results is to give examples of data for which $(\mathrm{RCD})_{I}$ has a branch of solutions at $+\infty$.

We consider the problem (5.6)-(5.8). We scale this problem as follows (see also [15]). We set

$$
s=\lambda^{-2} \bar{s}, J=\lambda^{-2} \bar{J}, \lambda^{2}=I^{-1 / 2} .
$$

Then (5.6)-(5.8) becomes

$$
\begin{gathered}
\bar{s}^{\prime}=\frac{\lambda^{4} N N^{\prime}}{\bar{s}}-\frac{N}{\bar{s}}+2 \bar{J} \quad \text { on } \Omega_{M}, \\
\bar{s}(x)=\lambda^{2} \sqrt{N^{2}(x)+4 \delta^{4}}, \quad x=0,1, \\
{\left[\bar{s}^{2}-\lambda^{4} N^{2}\right]_{a_{i}}=0 \quad \forall i \in\{1, \ldots, M\} .}
\end{gathered}
$$

We set

$$
\bar{s}=\sqrt{u} .
$$

Moreover, to simplify the notation we still denote by $J$ the scaled quantity $\bar{J}$. Then (6.2)-(6.4) becomes

$$
\begin{aligned}
& \frac{u^{\prime}}{2}=\lambda^{4} N N^{\prime}-N+2 J \sqrt{u} \text { on } \Omega_{M}, \\
& u(x)=\lambda^{4}\left(N^{2}(x)+4 \delta^{4}\right), \quad x=0,1,
\end{aligned}
$$




$$
\left[u-\lambda^{4} N^{2}\right]_{a_{i}}=0 \quad \forall i \in\{1, \ldots, M\} .
$$

For a given solution (if it exists) of (6.6)-(6.8) we set

$$
u_{i}^{ \pm}=u\left(a_{i}^{ \pm}\right), \quad i \in\{0, \ldots, M+1\},
$$

and

$$
U=\left(u_{1}^{-}, u_{1}^{+}, \ldots, u_{M}^{-}, u_{M}^{+}, J\right)
$$

We know from (5.17) that

$$
\int_{0}^{1} N(t) d t \neq 0 \Longrightarrow J \neq 0 .
$$

We start with the following result.

Proposition 6.1. Assume that

$$
N_{\mid\left[a_{i}, a_{i+1}\right]}=N_{i} \in \mathbb{R} \text { for } i \in\{0, \ldots, M\} \text { and } \int_{0}^{1} N(t) d t \neq 0,
$$

and let $(u, J)$ be a solution of (6.6)-(6.8) such that $u \in \mathcal{C}\left(\bar{\Omega}, \mathcal{P}_{M}\right)$ and $u>0$ on $\bar{\Omega}$. Then for every $i \in\{0, \ldots, M\}$,

$$
u_{\mid\left[a_{i}, a_{i+1}\right]}<\frac{N_{i}}{2 J} \text {, or } u_{\mid\left[a_{i}, a_{i+1}\right]}>\frac{N_{i}}{2 J} \text {, or } u_{\mid\left[a_{i}, a_{i+1}\right]}=\frac{N_{i}}{2 J} .
$$

Proof. We remark that, since $N$ is constant on $\left[a_{i}, a_{i+1}\right]$,

$$
\left(\frac{N_{i}}{2 J}\right)^{2} \quad \text { is a solution of }(6.6) \text { if } \frac{N_{i}}{2 J}>0 .
$$

Hence from the Cauchy-Lipschitz uniqueness theorem we easily conclude our proof.

We get the following result from an easy integration of (6.6) for piecewise constant functions $N$.

Proposition 6.2. Assume the hypotheses of proposition 6.1 and let $(u, J)$ be given as in proposition 6.1. We define $U$ as in (6.9)-(6.10). Then $U$ satisfies

$$
G(U, \lambda)=0,
$$

where $G$ is the map defined from $(0,+\infty)^{2 M} \times \mathbb{R}^{\star} \times \mathbb{R}$ into $\mathbb{R}^{2 M+1}$ by

$$
G(U, \lambda)=\left(G_{0}(U, \lambda), \ldots, G_{2 M}(U, \lambda)\right),
$$

with (for $i \in\{0, \ldots, M\})$

$$
\begin{gathered}
G_{i}(U, \lambda)=\exp \left(2 J N_{i}^{-1}\left(\sqrt{u_{i+1}^{-}}-\sqrt{u_{i}^{+}}\right)-4 J^{2} N_{i}^{-1}\left(a_{i+1}-a_{i}\right)\right) \\
\left(\sqrt{u_{i+1}^{-}}-N_{i}(2 J)^{-1}\right)-\left(\sqrt{u_{i}^{+}}-N_{i}(2 J)^{-1}\right) \text { if } N_{i} \neq 0, \\
G_{i}(U, \lambda)=\sqrt{u_{i+1}^{-}}-\sqrt{u_{i}^{+}}-2 J\left(a_{i+1}-a_{i}\right) \text { if } N_{i}=0, \\
G_{i}(U, \lambda)=u_{i-M}^{+}-u_{i-M}^{-}-\lambda^{4}\left[N^{2}\right]_{a_{i-M}} \text { for } i \in\{M+1, \ldots, 2 M\} .
\end{gathered}
$$


We set

$$
g(y)=e^{y}(y-1), g_{i}=g_{\mid B_{i}} i=0,1, B_{0}=\mathbb{R}^{-}, B_{1}=\mathbb{R}^{+} .
$$

We set

$$
C_{i}=g\left(B_{i}\right), r_{i}=g_{i}^{-1} \quad i=0,1
$$

Assume now that $U=\left(u_{1}^{-}, u_{1}^{+}, \ldots, u_{M}^{-}, u_{M}^{+}, J\right)$ is given in $(0,+\infty)^{2 M} \times \mathbb{R}^{\star}$ such that (6.13) holds, where $G$ is the map defined by (6.14)-(6.18). Let $i \in\{0, \ldots, M\}$ be given and assume first that $N_{i} \neq 0$. We set

$$
y_{i}^{+}=\frac{2 J \sqrt{u_{i}^{+}}}{N_{i}}, y_{i+1}^{-}=\frac{2 J \sqrt{u_{i+1}^{-}}}{N_{i}} .
$$

If $N_{i}<0$, we define $j$ as the integer in $\{0,1\}$ such that $y_{i}^{+} \in B_{j}$. We then set

$$
y(x)=r_{j}\left(g_{j}\left(y_{i}^{+}\right) \exp \left(\frac{4 J^{2}\left(x-a_{i}\right)}{N_{i}}\right)\right) \quad \forall x \in\left[a_{i}, a_{i+1}\right] .
$$

If $N_{i}>0$, we define $j$ as the integer in $\{0,1\}$ such that $y_{i+1}^{+} \in B_{j}$. We then set

$$
y(x)=r_{j}\left(g_{j}\left(y_{i+1}^{-}\right) \exp \left(\frac{4 J^{2}\left(x-a_{i+1}\right)}{N_{i}}\right)\right) \quad \forall x \in\left[a_{i}, a_{i+1}\right] .
$$

For both cases we set

$$
u(x)=\left(\frac{N_{i} y(x)}{2 J}\right)^{2} .
$$

Assume now that $N_{i}=0$. Then we set

$$
\begin{gathered}
u(x)=\left(\sqrt{u_{i}^{+}}+2 J\left(x-a_{i}\right)\right)^{2} \quad \forall x \in\left[a_{i}, a_{i+1}\right] \text { if } J \geq 0, \\
u(x)=\left(\sqrt{u_{i+1}^{-}}+2 J\left(x-a_{i+1}\right)\right)^{2} \quad \forall x \in\left[a_{i}, a_{i+1}\right] \text { if } J \leq 0,
\end{gathered}
$$

Of course we have

$$
u>0 \text { on }[0,1]
$$

Moreover it is easy to check that $u \in \mathcal{C}^{1}\left(\bar{\Omega}, \mathcal{P}_{M}\right)$ and that $(u, J)$ is a solution of (6.6)-(6.8). Hence we have proved the following result.

Proposition 6.3. Assume the hypotheses of proposition 6.1 and let $U$ be given in $(0,+\infty)^{2 M} \times \mathbb{R}^{\star}$ such that (6.13) holds. Let $u$ be defined as above. Then $(u, J)$ is a solution of (6.6)-(6.8) with $u \in \mathcal{C}^{1}\left(\bar{\Omega}, \mathcal{P}_{M}\right)$ and $u>0$.

If we formally set $\lambda=0$ in (6.6)-(6.8), we obtain the limit problem

$$
\begin{aligned}
& \frac{u^{\prime}}{2}=-N+2 J \sqrt{u}, \\
& u(x)=0, \quad x=0,1 .
\end{aligned}
$$

Lemma 6.4. Assume the hypotheses of proposition 6.1. Assume moreover that $N_{0} \neq 0, N_{M} \neq 0$ and that there exists $\left(u^{0}, J^{0}\right)$ with $u^{0} \in \mathcal{C}^{1}\left(\bar{\Omega}, \mathcal{P}_{M}\right)$ and $u^{0}>0$ on $(0,1), J^{0} \in \mathbb{R}$ such that $\left(u^{0}, J^{0}\right)$ is a solution of (6.22)-(6.23). Then the following properties hold:

$$
\begin{gathered}
\left(u^{0}\right)^{-1 / 2} \in L^{1}(0,1), \\
G\left(U^{0}, 0\right)=0,
\end{gathered}
$$


and $\partial_{U} G\left(U^{0}, 0\right)$ is boundedly invertible, where

$$
U^{0}=\left(u^{0}\left(a_{1}^{-}\right), u^{0}\left(a_{1}^{+}\right), \ldots, u^{0}\left(a_{M}^{-}\right), u^{0}\left(a_{M}^{+}\right), J^{0}\right) .
$$

Proof. Since $\left(u^{0}, J^{0}\right)$ is a solution of $(6.22)-(6.23)$, we have

$$
\left(\sqrt{u^{0}}\right)^{\prime}=-\frac{N_{i}}{\sqrt{u^{0}}}+2 J^{0} \quad \text { in }\left[a_{i}, a_{i+1}\right] .
$$

Hence since $N_{0} \neq 0$ and $N_{M} \neq 0$, we deduce that $\left(u^{0}\right)^{-1 / 2}$ is integrable in a neighbourhood of 0 and 1 . Therefore (6.24) is proved. Moreover (6.25) trivially holds from our assumptions. Let $W=\left(w_{1}^{-}, w_{1}^{+}, \ldots, w_{M}^{-}, w_{M}^{+}, j\right)$ be such that

$$
\partial_{U} G\left(U^{0}, 0\right) . W=0 \text {. }
$$

We have

$$
u_{i}^{0,+}=u_{i}^{0,-}=u_{i}^{0}
$$

From (6.18) and (6.26) we can set

$$
w_{i}=w_{i}^{-}=w_{i}^{+}
$$

For $U=\left(u_{1}^{-}, u_{1}^{+}, \ldots, u_{M}^{-}, u_{M}^{+}, J\right)$ and for $i \in\{1, \ldots, M\}$ such that $\sqrt{u_{i}^{+}} \neq \frac{N_{i}}{2 J}$, $\sqrt{u_{i+1}^{-}} \neq \frac{N_{i}}{2 J}$, we set

$$
H_{i}(U, \lambda)=\sqrt{u_{i+1}^{-}}-\sqrt{u_{i}^{+}}+\frac{N_{i}}{2 J} \log \left(\frac{\sqrt{u_{i+1}^{-}}-\frac{N_{i}}{2 J}}{\sqrt{u_{i}^{+}}-\frac{N_{i}}{2 J}}\right)-2 J\left(a_{i+1}-a_{i}\right) .
$$

Express $G_{i}(U, \lambda)$ in terms of $H_{i}(U, \lambda)$. Differentiating the resulting equality with respect to $U$, we deduce that

$$
\partial_{U} H_{i}\left(U^{0}, 0\right) . W=0 .
$$

We then denote by $(w, r), r \in \mathbb{R}$, the solution of

$$
\frac{w^{\prime}}{2}=\frac{J^{0} w}{\sqrt{u^{0}}}+2 r \sqrt{u^{0}} \text { in } \Omega
$$

and

$$
w(0)=w(1)=0 .
$$

Using the equation $\partial_{U} H_{i}\left(U^{0}, 0\right) . W=0$ one can then check that $w\left(a_{i}\right)=w_{i}$ and $r=j$ hold. On the other hand, since (6.24) holds we deduce that $(w, j)=(0,0)$, so that $W=0$. This proves that $\partial_{U} G\left(U^{0}, 0\right)$ is boundedly invertible.

This lemma, together with the implicit function theorem and proposition 6.3 , implies the following corollary.

Corollary 6.5. Assume the hypotheses of lemma 6.4. Assume moreover that there exists a solution $\left(U^{0}, J^{0}\right)$ of (6.22)-(6.23). Then there exists $\lambda_{0}>0$ such that for every $\lambda \in\left[0, \lambda_{0}\right)$, problem (6.6)-(6.8) has a solution $(u(., \lambda), J(\lambda))$ which depends in $a \mathcal{C}^{1}$ way on $\lambda$ and satisfies $u(., \lambda) \in \mathcal{C}^{1}\left(\overline{\Omega, \mathcal{P}_{M}}\right), u(., \lambda)>0$ and $(u(., 0), J(0))=$ $\left(u^{0}, J(0)\right)$. 
Lemma 6.6. Assume the hypotheses of corollary 6.5, and assume in addition that $N_{0}<0$ and $N_{M}>0$. Then there exists $\lambda_{1} \in\left(0, \lambda_{0}\right)$ such that for every $\lambda \in\left(0, \lambda_{1}\right)$ the solution $(u(., \lambda), J(\lambda))$ defined in corollary 6.5 satisfies

$$
u(., \lambda)>\lambda^{4}|N(.)|^{2} .
$$

Proof. We set

$$
\phi_{\lambda}(x)=u(x, \lambda)-\lambda^{4} N^{2}(x) .
$$

Since (6.7) holds we have $\phi_{\lambda}(0)>0$. We claim that $\phi_{\lambda}>0$ on $\left[0, a_{1}\right]$. Assume to the contrary that this is not true. We denote by $x_{0}$ the first point (starting from $x=0)$ at which $\phi_{\lambda}$ vanishes. Then since (6.6)-(6.8) holds, we have

$$
\partial_{x} u\left(x_{0}, \lambda\right)=-2 N_{0}+4 J(\lambda) \lambda^{2}\left|N_{0}\right| .
$$

Since $J(\lambda)$ converges to $J^{0}$ as $\lambda$ goes to 0 and since $N_{0}<0$, we deduce from the last inequality that $\partial_{x} \phi_{\lambda}\left(x_{0}\right)>0$ for $\lambda$ sufficiently small. This implies that $\phi_{\lambda}<0$ in a left neighbourhood of $x_{0}$, which contradicts the definition of $x_{0}$ since $\phi_{\lambda}(0)>0$. We prove in a similar way that $\phi_{\lambda}>0$ on $\left.\left[a_{M}, 1\right]\right]$. Hence we obtain that $u(., \lambda)>\lambda^{4}|N(.)|^{2}$ on $\left[0, a_{1}\right] \cup\left[a_{M}, 1\right]$. We now set $\eta=2^{-1} \inf _{\left[a_{1}, a_{M}\right]}\left(\sqrt{u^{0}}\right)$. Of course $\eta>0$. On the other hand we know from corollary 6.5 that

$$
\|\sqrt{u(., \lambda)}-\sqrt{u(., 0)}\|_{\infty,\left[a_{i}, a_{i+1}\right]} \rightarrow 0
$$

as $\lambda \rightarrow 0$. Hence for $\lambda$ sufficiently small we deduce that $\sqrt{u(., \lambda)} \geq \eta \geq \lambda^{2}|N(x)|$ for all $x \in \Omega$.

We now turn our attention to the analysis of the limit problem (6.22)-(6.23). For the sake of simplicity we will assume from now on that

$$
\int_{0}^{1} N(t) d t>0
$$

We set

$$
\begin{gathered}
z(y)=J \sqrt{u(x)}, \\
D(y)=N(x),
\end{gathered}
$$

where

$$
y=x J^{2} .
$$

Then the problem (6.22)-(6.23) becomes

$$
\begin{gathered}
z^{\prime}(y)=-\frac{D(y)}{z(y)}+2, \quad y \in(0, a), \\
z(0)=z(a)=0,
\end{gathered}
$$

where

$$
a=J^{2}
$$

where $a$ is now unknown and where' ${ }^{\prime}$ denotes the differentiation with respect to $y$. We set

$$
\overline{\mathcal{P}}_{M}=\left(b_{i}\right)_{0 \leq i \leq M+1}, \tilde{\Omega}=(0, a), b_{i}=a a_{i} \quad \forall i \in\{0, \ldots, M+1\} .
$$

Hence we have proved the following result. 
Lemma 6.7. Assume that $N \in W^{1, \infty}\left(\Omega, \mathcal{P}_{M}\right)$ and that (6.30) holds. Then the limit problem (6.22)-(6.23) has a solution $(u, J) \in \mathcal{C}^{1}\left(\bar{\Omega}, \mathcal{P}_{M}\right) \times(0,+\infty), u>0$ on $\Omega$, if and only if the scaled problem $(6.34)-(6.35)$ has a solution $(z, a) \in \mathcal{C}^{1}\left(\bar{\Omega}, \mathcal{P}_{M}\right) \times$ $(0,+\infty), z>0$ on $\tilde{\Omega}$.

Our purpose now is to give a constructive method for giving examples of doping functions $N$ and partitions $\mathcal{P}_{M}$ for which the reduced current driven equations have a branch of solutions at infinity. We proceed as follows.

We set

$$
f(x)=x+\log (|1-x|), \quad f_{i}=f_{\mid A_{i}}, \quad i \in\{0,1,2\},
$$

and

$$
\begin{gathered}
A_{0}=(-\infty, 0), A_{1}=(0,1), A_{2}=(1,+\infty) . \\
E=\left\{\left(D, \tilde{\mathcal{P}}_{M}\right), \tilde{\mathcal{P}}_{M}=\left(b_{i}\right)_{0 \leq i \leq M+1},\right. \\
\left.b_{0}=0, D_{\mid\left[b_{i}, b_{i+1}\right]}=D_{i} \in \mathbb{R}, \operatorname{sign}\left(D_{i}\right)=(-1)^{i+1}, \forall i \in\{0, \ldots, M\}\right\}
\end{gathered}
$$

We then set $w_{0}=0$ and $t_{0}=-1$, and

$$
\begin{aligned}
& \mathcal{T}(M)= \\
& \left\{w=\left(w_{1}, \ldots, w_{M}, t_{1}, \ldots, t_{M}\right) \in \mathbb{R}^{2 M},(-1)^{i} w_{i}>0, t_{i}<0 \forall i \in\{1, \ldots, M\},\right. \\
& \quad\left(w_{i+1}-1\right)\left(w_{i} t_{i}^{-1}-1\right)>0 \forall i \in\{0, \ldots, M\} \\
& \quad(-1)^{i+1}\left(f_{j(i)}\left(w_{i+1}\right)-f_{j(i)}\left(w_{i} t_{i}^{-1}\right)\right)>0 \text { if } w_{i+1} \neq 1, \\
& \left.\quad \text { and } w_{i+1}=w_{i}=1 \text { if } w_{i+1}=1\right\},
\end{aligned}
$$

where $j(i)$ is the integer in $\{0,1,2\}$ such that $w_{i+1} \in A_{j}$ if $w_{i+1} \neq 1$.

Theorem 6.8. Let $M \geq 1$ be given. Then there exists $\left(D, \tilde{\mathcal{P}}_{M}\right) \in E$ such that (6.34)-(6.35) has a solution $(z, a) \in \mathcal{C}^{1}\left(\tilde{\Omega}, \tilde{\mathcal{P}}_{M}\right) \times(0,+\infty)$ with $z>0$ on $(0, a)$ (where $a=b_{M+1}$ ), if and only if $\mathcal{T}(M) \neq \emptyset$. Moreover if $\mathcal{T}(M) \neq \emptyset$, then the set of $\left(D, \tilde{\mathcal{P}}_{M}\right) \in E$ for which (6.34)-(6.35) has a solution is characterized by

$$
\left(D, \tilde{\mathcal{P}}_{M}\right)=T\left(w, D_{0}\right), \quad w \in \mathcal{T}(M), D_{0}<0,
$$

where $T$ is defined by (6.39)-(6.41).

Proof. Assume first that $\left(D, \tilde{\mathcal{P}}_{M}\right) \in E$ is such that (6.34)-(6.35) has a solution $(z, a) \in \mathcal{C}^{1}\left(\tilde{\Omega}, \tilde{\mathcal{P}}_{M}\right) \times(0,+\infty)$ with $z>0$ on $(0, a)$. Integration of (6.34) yields

$$
z_{i+1}-z_{i}+\frac{D_{i}}{2} \log \left(\frac{\left|z_{i+1}-D_{i} / 2\right|}{\left|z_{i}-D_{i} / 2\right|}=2\left(b_{i+1}-b_{i}\right) \quad \text { if } z_{i} \neq D_{i} / 2\right.
$$

and,

$$
z_{i+1}=z_{i} \quad \text { if } z_{i}=D_{i} / 2
$$

where we set $z_{j}=z\left(b_{j}\right)$. Since $D_{i} \neq 0$ we can set

$$
w_{i}=2 z_{i} D_{i-1}^{-1}, t_{i}=D_{i} D_{i-1}^{-1} \quad \forall i \in\{1, \ldots, M\}, w_{0}=0, t_{0}=-1 .
$$

We deduce from proposition 6.1 that we have $\left(w_{i+1}-1\right)\left(w_{i} t_{i}^{-1}-1\right)>0$. This together with the definition of the functions $f_{j}$ implies that $w=\left(w_{1}, \ldots, t_{M}\right) \in$ $\mathcal{T}(M)$. 
Conversely, assume that $\mathcal{T}(M) \neq \emptyset$. Let $D_{0}<0$ be given arbitrarily and consider $w=\left(w_{1}, \ldots, t_{M}\right) \in \mathcal{T}(M)$. Then, for $i \in\{0, \ldots, M\}$ such that $w_{i+1} \neq 1$ we set

$$
D_{i}=D_{0} \prod_{j=1}^{i} t_{j}
$$

and,

$$
\Delta_{i}=\frac{D_{i}}{4}\left(f_{j(i)}\left(w_{i+1}\right)-f_{j(i)}\left(w_{i} t_{i}^{-1}\right)\right)
$$

Then one can check that $\Delta_{i}>0$.

$$
\text { For } i \text { such that } w_{i}=1 \text { we choose } \Delta_{i}>0 \text { arbitrarily. }
$$

We then set for every $i \in\{1, \ldots, M\}$

$$
z_{i}=\frac{D_{i-1} w_{i}}{2}, b_{i+1}=b_{i}+\Delta_{i}, b_{0}=0, a=\sum_{i=0}^{M} \Delta_{i}
$$

We then construct $z$ as in proposition 6.3 (the case where $\lambda=0$ ).

\section{MUltipliCity RESUltS FOR FORWARD BIASED THYRISTORS}

For the sake of simplicity we restrict our analysis to the case of thyristors, i.e. $M=3$. Then we have the following result (whose easy proof is left to the reader).

\section{Lemma 7.1.}

$$
\begin{aligned}
\mathcal{T}(3) \supset A=\{ & \left(w_{1}, \ldots, t_{3}\right) \in \mathbb{R}^{6}, t_{1}<w_{1}<0, \\
& \left.0<w_{2}<w_{1} t_{1}^{-1}, t_{3}<w_{3}<w_{2} t_{2}^{-1}<0\right\} .
\end{aligned}
$$

Hence from our previous results, we deduce that for $w \in A$ and $D_{0}<0,(\mathrm{RCD})_{I}$ has a branch of solutions at $+\infty$ for $\left(D, \tilde{\mathcal{P}}_{M}\right)=T\left(w, D_{0}\right)$. Hence we assume from now on that $\left(D, \tilde{\mathcal{P}}_{M}\right)=T\left(w, D_{0}\right)$ with $w \in A$ and $D_{0}<0$. We now have to combine these results with the ones we gave previously (see theorem 5.4) for the existence of at least one saturation current to guarantee the existence of at least two positive saturation currents. We proceed as follows. We first remark that since we assumed that $\int_{0}^{1} N(t) d t>0$, and since $\left(D, \tilde{\mathcal{P}}_{M}\right)$ is such that $(\mathrm{RCD})_{I}$ has a branch of solutions at $+\infty$, we have $\min _{[0,1]}\left(\int_{x}^{1} N(t) d t \geq 0\right.$. Hence the condition $\min _{[0,1]}(F(., N))<0$ is sufficient (see theorem 5.4) for the existence of at least one positive current.

We first remark that

$$
\min _{[0,1]}(F(., N))<0 \Longleftrightarrow \min _{[0, a]}(\tilde{F}(., D))<0,
$$

where

$$
\tilde{F}(y, D)=\int_{y}^{a} D(t) d t \int_{0}^{a}|D(t)| d t-\int_{0}^{a} D(t) d t \int_{y}^{a}|D(t)| d t
$$

Since $M=3$ and $\operatorname{sign}\left(D_{i}\right)=(-1)^{i+1}$, we deduce that $\min _{[0, a]}(\tilde{F}(., D))<0$ if and only if $\tilde{F}\left(b_{2}, D\right)<0$. Since $D$ is piecewise constant we deduce that $\min _{[0, a]}(\tilde{F}(., D))$ $<0$ if and only if

$$
D_{1} D_{2} \Delta_{1} \Delta_{2}-D_{0} D_{3} \Delta_{0} \Delta_{3}<0
$$


Since $(\mathrm{RCD})_{I}$ has a branch of solutions at $+\infty$, we deduce that there exist at least two positive saturation currents if the set

$$
B=\{w \in A, \tilde{K}(w)<0)\}
$$

is not empty, where

$$
\begin{aligned}
& \tilde{K}(w)=t_{1}^{2}\left(f_{1}\left(w_{2}\right)-f_{1}\left(w_{1} t_{1}^{-1}\right)\right) \\
& \times\left(f_{0}\left(w_{3}\right)-f_{0}\left(w_{2} t_{2}^{-1}\right)\right)+t_{3}^{2} f_{0}\left(w_{1}\right) f_{1}\left(w_{3} t_{3}^{-1}\right) .
\end{aligned}
$$

Hence we have proved the following result.

Theorem 7.2. Assume that the set

$$
B=\{w \in A, \tilde{K}(w)<0\} \neq \emptyset,
$$

where $\tilde{K}$ is defined by (7.4). Then for $w \in B$ and $\left(D, \tilde{\mathcal{P}}_{M}\right)=T\left(w, D_{0}\right)$, with $D_{0}<0$ chosen arbitrarily, there exists $\delta_{0}>0$ such that for every $\delta \in\left[0, \delta_{0}\right](R C D)_{I}$ has at least two positive saturation currents, so that the reduced voltage driven equations have at least three solutions in a subinterval of $\mathbb{R}^{+}$.

Lemma 7.3. Let $\alpha \in(0,1)$ be given. Then $B \supset B_{\alpha} \neq \emptyset$, where

$$
\begin{array}{r}
B_{\alpha}=\left\{w=\left(w_{1}, \ldots, t_{3}\right), t_{1}<0,0<w_{2}<\alpha, t_{3}<w_{3}<w_{2} t_{2}^{-1}<0,\right. \\
\left.w_{1} \in\left(t_{1}, \min \left(\alpha t_{1}, \theta(\hat{w})\right)\right)\right\},
\end{array}
$$

where $\hat{w}=\left(w_{2}, w_{3}, t_{1}, t_{2}, t_{3}\right)$ and $\theta$ is defined as in (7.11).

Proof. Let $\alpha \in(0,1)$ and $w \in A$ be given. We fix the parameters $\left(w_{2}, w_{3}, t_{1}, t_{2}, t_{3}\right)$ such that $t_{1}<0,0<w_{2}<\alpha$ and $t_{3}<w_{3}<w_{2} t_{2}^{-1}<0$, and we set $\hat{w}=$ $\left(w_{2}, w_{3}, t_{1}, t_{2}, t_{3}\right)$. Since $w \in A$ we must have in addition $0<w_{2}<w_{1} t_{1}^{-1}$. This occurs whenever $w_{1} \leq \alpha t_{1}$. Since $w_{1}>t_{1}$ also holds, $w_{1}$ takes values in $\left(t_{1}, \alpha t_{1}\right]$. We now remark that since $f_{0}$ is increasing on $\mathbb{R}^{-}$and since $f_{1}\left(w_{3} t_{3}^{-1}\right)<0$, we have

$$
\tilde{K}\left(w_{1}, \hat{w}\right)<L\left(w_{1}, \hat{w}\right)
$$

where

$$
\begin{aligned}
& L\left(w_{1}, \hat{w}\right)=t_{1}^{2}\left(f_{1}\left(w_{2}\right)-f_{1}\left(w_{1} t_{1}^{-1}\right)\right) \\
& \times\left(f_{0}\left(w_{3}\right)-f_{0}\left(w_{2} t_{2}^{-1}\right)\right)+t_{3}^{2} f_{0}\left(t_{1}\right) f_{1}\left(w_{3} t_{3}^{-1}\right) .
\end{aligned}
$$

Hence $\tilde{K}\left(w_{1}, \hat{w}\right)<0$ will hold if $L\left(w_{1}, \hat{w}\right) \leq 0$ holds. We set

$$
\beta(\hat{w})=t_{1}^{2}\left(f_{1}\left(w_{2}\right)\right)\left(f_{0}\left(w_{3}\right)-f_{0}\left(w_{2} t_{2}^{-1}\right)\right)+t_{3}^{2} f_{0}\left(t_{1}\right) f_{1}\left(w_{3} t_{3}^{-1}\right),
$$

and

$$
R_{\hat{w}}\left(w_{1}\right)=t_{1}^{2} f_{1}\left(w_{1} t_{1}^{-1}\right)\left(f_{0}\left(w_{3}\right)-f_{0}\left(w_{2} t_{2}^{-1}\right)\right) .
$$

Hence we have

$$
L\left(w_{1}, \hat{w}\right) \leq 0 \Longleftrightarrow \beta(\hat{w}) \leq R_{\hat{w}}\left(w_{1}\right) .
$$

We now remark that $R_{\hat{w}}$ is a strictly decreasing function of $w_{1}$ on $\left(t_{1}, \alpha t_{1}\right]$ and that it goes to $+\infty$ as $w_{1}$ goes to $t_{1}$. We also note that $\beta(\hat{w})>0$. Hence $L\left(w_{1}, \hat{w}\right) \leq 0$ if and only if $w_{1} \in\left(t_{1}, \min \left(\alpha t_{1}, \theta(\hat{w})\right)\right)$, where $\theta$ is given by

$$
\theta(\hat{w})=\left(R_{\hat{w}}\right)^{-1}(\beta(\hat{w}))
$$

We deduce from the above results and from corollary 3.5 the following multiplicity result for forward biased thyristors. 
Theorem 7.4. Let $\alpha \in(0,1)$ and $w \in B_{\alpha}$ be given arbitrarily. We set $\left(D, \tilde{\mathcal{P}}_{M}\right)=$ $T\left(w, D_{0}\right)$, where $D_{0}<0$ is chosen arbitrarily. Then there exists $\delta_{0}$, such that for all $\delta \in\left[0, \delta_{0}\right]$, there exists $\varepsilon_{2}=\varepsilon_{2}\left(D, \delta, \tilde{\mathcal{P}}_{M}\right)>0$ such that for all $\varepsilon \in\left(0, \varepsilon_{2}\right)$, there exist $\left(V_{-, \varepsilon}, V_{+, \varepsilon}\right) \in \mathbb{R}^{\nu} \times \mathbb{R}^{\nu}, V_{-, \varepsilon}<V_{+, \varepsilon}$ such that $(V D)_{\bar{V}, \varepsilon}$ has at least three solutions in $\left(H^{1}(\Omega)\right)^{3} \times \mathbb{R}^{2}$ for all $\bar{V} \in\left(V_{-, \varepsilon}, V_{+, \varepsilon}\right)$.

It is important to remark that the constructive method given above is general and allows us to give other examples of multiplicity (for a higher number of $p n$ junctions).

\section{ACKNOWLEDGMENT}

I would like to thank Professor Denis Serre for a fruitful discussion while I was visiting the E.N.S. of Lyon.

\section{REFERENCES}

[1] F. Alabau, Uniform asymptotic error estimates for the semiconductor device and electrochemistry equations, Nonlinear Anal. 14 (1990), 123-139. MR 92i:34063

[2] _ A method for proving uniqueness theorems for the stationary semiconductor device and electrochemistry equations, Nonlinear Anal. 18 (1992), 861-872. MR 93d:78006

[3] _ New uniqueness theorems for the one-dimensional drift-diffusion semiconductor device equations, Siam J. Math. Anal. (1995), 715-737. MR 96c:78008

[4] _ Structural properties of the one-dimensional drift-diffusion models for semiconductors, Trans. on the A.M.S. 348 (1996), 823-871. MR 96i:82157

[5] _ Uniqueness results for the steady-state electrodiffusion equations in case of monotonic potentials and multiple junctions, Nonlinear Anal. 29 (1997), 849-887. CMP 97:14

[6] F. Alabau and M. Moussaoui, Asymptotic estimates for the multi-dimensional electrodiffusion equations, M3AS, To appear.

[7] P.C. Fife, Semilinear elliptic boundary value problems with small parameters, Arch. Rational Mech. 52 (1974), 205-232. MR 51:10863

[8] A. Friedman, Elliptic and parabolic systems associated with semiconductor modeling, Progress in partial differential equations: elliptic and parabolic problems, vol. 266, Pitman Research Notes in Mathematics Series, 1992, pp. 17-23. MR 93f:35007

[9] R.E. O'Malley jr., Introduction to singular perturbations, Academic Press, New York, 1974. MR 53:6038

[10] P.A. Markowich, The stationary semiconductor device equations, Springer, Wien-New York, 1986. MR 87b:78042

[11] M.S. Mock, On equations describing steady-state carrier distributions in a semiconductor device, Comm. Pure Appl. Math. 25 (1972), 781-792. MR 48:1591

[12] _ An example of nonuniqueness of stationary solutions in semiconductor device models, Compel 1 (1982), 165-174.

[13] _ Analysis of mathematical models of semiconductor devices, Boole Press, Dublin, 1983. MR 84m:78002

[14] W.V. Van Roosbroeck, Theory of flow of electrons and holes in germanium and other semiconductors, Bell Syst. Techn. J. 29 (1950), 560-607.

[15] I. Rubinstein, Electro-diffusion of ions, Siam Studies in Applied Mathematics, 1990. MR 91m:78019

[16] T. I. Seidman, Steady-state solutions of diffusion-reaction systems with electrostatic convection, Nonlinear Anal. 4 (1980), 623-637. MR 81i:35075

[17] H. Steinruck, A bifurcation analysis of the one-dimensional steady-state semiconductor device equations, Siam J. Appl. Math. 49 (1989), 1102-1121. MR 90h:94066

[18] S.M. Sze, Physics of semiconductor devices, John Wiley \& Sons, New York, 1969.

I.R.M.A., Université Louis Pasteur, 7, rue René Descartes, 67084 Strasbourg Cedex (FrANCE)

E-mail address: alabau@math.u-strasbg.fr 